\title{
AN INTERESTING CLASS OF OPERATORS WITH UNUSUAL SCHATTEN-VON NEUMANN BEHAVIOR
}

\author{
A.B. ALEKSANDROV, S. JANSON, V.V. PELLER AND R. ROCHBERG
}

We dedicate this paper to Jaak Peetre on occasion of his 65th birthday and to the memory of Tom Wolff. Both helped shape the mathematics of our time and profoundly influenced our mathematical thoughts. Each, through his singular humanity, helped our hearts grow.

Abstract. We consider the class of integral operators $Q_{\varphi}$ on $L^{2}\left(\mathbb{R}_{+}\right)$of the form $\left(Q_{\varphi} f\right)(x)=\int_{0}^{\infty} \varphi(\max \{x, y\}) f(y) d y$. We discuss necessary and sufficient conditions on $\varphi$ to insure that $Q_{\varphi}$ is bounded, compact, or in the Schatten-von Neumann class $\boldsymbol{S}_{p}, 1<p<\infty$. We also give necessary and sufficient conditions for $Q_{\varphi}$ to be a finite rank operator. However, there is a kind of cut-off at $p=1$, and for membership in $\boldsymbol{S}_{p}, 0<p \leq 1$, the situation is more complicated. Although we give various necessary conditions and sufficient conditions relating to $Q_{\varphi} \in \boldsymbol{S}_{p}$ in that range, we do not have necessary and sufficient conditions. In the most important case $p=1$, we have a necessary condition and a sufficient condition, using $L^{1}$ and $L^{2}$ modulus of continuity, respectively, with a rather small gap in between. A second cut-off occurs at $p=1 / 2$ : if $\varphi$ is sufficiently smooth and decays reasonably fast, then $Q_{\varphi}$ belongs to the weak Schatten-von Neumann class $\boldsymbol{S}_{1 / 2, \infty}$, but never to $\boldsymbol{S}_{1 / 2}$ unless $\varphi=0$.

We also obtain results for related families of operators acting on $L^{2}(\mathbb{R})$ and $\ell^{2}(\mathbb{Z})$.

We further study operations acting on bounded linear operators on $L^{2}\left(\mathbb{R}_{+}\right)$ related to the class of operators $Q_{\varphi}$. In particular we study Schur multipliers given by functions of the form $\varphi(\max \{x, y\})$ and we study properties of the averaging projection (Hilbert-Schmidt projection) onto the operators of the form $Q_{\varphi}$

\section{Contents}

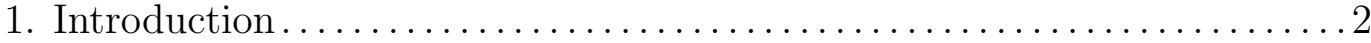

2. Preliminaries ........................................... 6

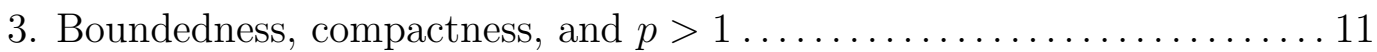

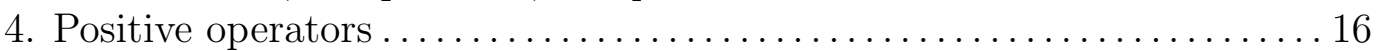

5. A sufficient condition, $1 / 2<p \leq 1 \ldots \ldots \ldots \ldots \ldots \ldots \ldots \ldots \ldots \ldots \ldots \ldots \ldots \ldots \ldots \ldots$

Date: April 15, 2001; revised October 4, 2001.

The first author is partially supported by Grant 99-01-00103 of Russian Foundation of Fundamental Studies and by Grant 326.53 of Integration. The third author is partially supported by NSF grant DMS 9970561. The fourth author is partially supported by NSF grant DMS 9970366. 


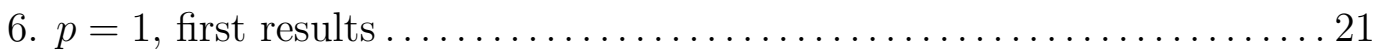

7. Schur multipliers of the form $\psi(\max \{x, y\}), x, y \in \mathbb{R}_{+} \ldots \ldots \ldots \ldots 26$

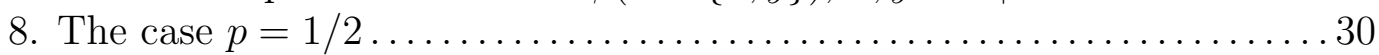

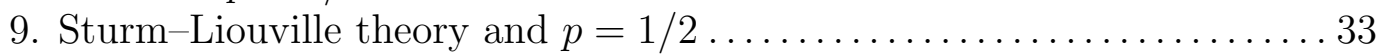

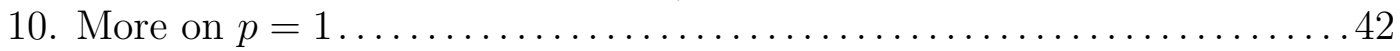

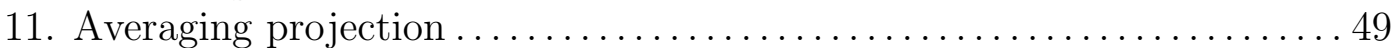

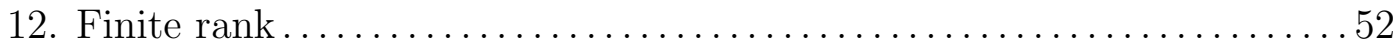

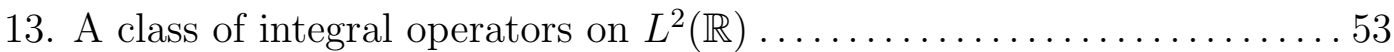

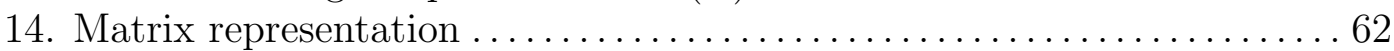

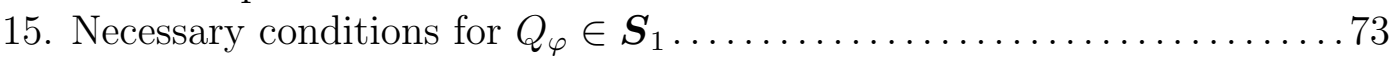

16. Dilation of Symbols ................................... 84

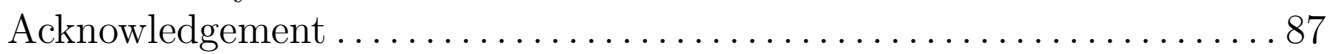

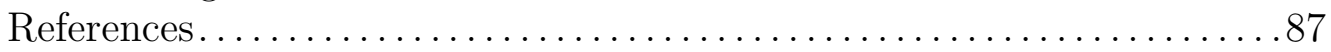

\section{Introduction}

For a function $\varphi \in L_{\text {loc }}^{1}\left(\mathbb{R}_{+}\right)$, which means that $\varphi$ is a locally integrable function on $\mathbb{R}_{+}=(0, \infty)$, we define the operator $Q_{\varphi}$ on the set of bounded compactly supported functions $f$ in $L^{2}\left(\mathbb{R}_{+}\right)$by

$$
\left(Q_{\varphi} f\right)(x)=\int_{0}^{\infty} \varphi(\max \{x, y\}) f(y) d y
$$

equivalently,

$$
\left(Q_{\varphi} f\right)(x)=\varphi(x) \int_{0}^{x} f(y) d y+\int_{x}^{\infty} \varphi(y) f(y) d y .
$$

We are going to study when $Q_{\varphi}$ is (i.e., extends to) a bounded operator in $L^{2}\left(\mathbb{R}_{+}\right)$, and when this operator is compact, or belongs to Schatten-von Neumann classes $\boldsymbol{S}_{p}$. by

We will also consider the corresponding Volterra operators $Q_{\varphi}^{+}$and $Q_{\varphi}^{-}$defined

$$
\begin{aligned}
& \left(Q_{\varphi}^{+} f\right)(x)=\varphi(x) \int_{0}^{x} f(y) d y, \\
& \left(Q_{\varphi}^{-} f\right)(x)=\int_{x}^{\infty} \varphi(y) f(y) d y
\end{aligned}
$$

thus $Q_{\varphi}=Q_{\varphi}^{+}+Q_{\varphi}^{-}$.

It is straightforward to see (and proved more generally in Theorem 2.4) that if any of these three operators is bounded on $L^{2}\left(\mathbb{R}_{+}\right)$, then $\int_{a}^{\infty}|\varphi|^{2}<\infty$ for any 
$a>0$, and thus the integrals above converge for any $f \in L^{2}\left(\mathbb{R}_{+}\right)$and define all three operators on $L^{2}\left(\mathbb{R}_{+}\right)$.

We find in $\S 3$ simple necessary and sufficient conditions for $Q_{\varphi}$ to be bounded or compact, and for $Q_{\varphi} \in \boldsymbol{S}_{p}, 1<p<\infty$. The conditions are $\varphi \in X_{\infty}, \varphi \in X_{\infty}^{0}$ and $\varphi \in X_{p}$, respectively, where the spaces $X_{p}, X_{\infty}$, and $X_{\infty}^{0}$ are defined as follows.

Definition. If $0<p<\infty$, let $X_{p}$ be the linear space of all measurable functions on $\mathbb{R}_{+}$that satisfy the equivalent conditions

$$
\begin{aligned}
& \sum_{n \in \mathbb{Z}} 2^{n p / 2}\left(\int_{2^{n}}^{2^{n+1}}|\varphi(x)|^{2} d x\right)^{p / 2}<\infty \\
& \sum_{n \in \mathbb{Z}} 2^{n p / 2}\left(\int_{2^{n}}^{\infty}|\varphi(x)|^{2} d x\right)^{p / 2}<\infty ; \\
& x^{1 / 2}\left(\int_{x}^{\infty}|\varphi(y)|^{2} d y\right)^{1 / 2} \in L^{p}(d x / x) .
\end{aligned}
$$

Similarly, let $X_{\infty}$ be the linear space of all measurable functions on $\mathbb{R}_{+}$that satisfy the equivalent conditions

$$
\begin{gathered}
\sup _{n \in \mathbb{Z}} 2^{n}\left(\int_{2^{n}}^{2^{n+1}}|\varphi(x)|^{2} d x\right)<\infty \\
\sup _{x>0} x \int_{x}^{\infty}|\varphi(y)|^{2} d y<\infty .
\end{gathered}
$$

Let $X_{\infty}^{0}$ be the subspace of $X_{\infty}$ consisting of the functions that satisfy the equivalent conditions

$$
\begin{gathered}
\lim _{n \rightarrow \pm \infty} 2^{n}\left(\int_{2^{n}}^{2^{n+1}}|\varphi(x)|^{2} d x\right)=0 \\
\lim _{x \rightarrow 0} x \int_{x}^{\infty}|\varphi(y)|^{2} d y=\lim _{x \rightarrow \infty} x \int_{x}^{\infty}|\varphi(y)|^{2} d y=0 .
\end{gathered}
$$

The equivalence of the different conditions is an exercise. For $1 \leq p \leq \infty, X_{p}$ is a Banach space with the norm

$$
\|\varphi\|_{X_{p}}=\left\|x^{1 / 2}\left(\int_{x}^{\infty}|\varphi(y)|^{2} d y\right)^{1 / 2}\right\|_{L^{p}(d x / x)} ;
$$

for $0<p<1$, this is a quasi-norm and $X_{p}$ is a quasi-Banach space. $X_{\infty}^{0}$ is a closed subspace of $X_{\infty}$, and thus a Banach space too. Note that $X_{p} \subset X_{q}$ if $0<p \leq q \leq \infty$. 
Remark. It is well known [Pee] that $\varphi \in X_{p}$ if and only if the Fourier transform $\mathcal{F} \varphi$ belongs to the homogeneous Besov space $B_{2 p}^{1 / 2}$ (here we identify $\varphi$ with the function extended to $\mathbb{R}$ by zero on $\mathbb{R}_{-}$).

Note that the operators $Q_{\varphi}$ appear in a natural way in [MV] when studying the boundedness problem for the Sturm-Liouville operator $\mathcal{L}$ from $\stackrel{\circ}{L_{2}^{1}}\left(\mathbb{R}_{+}\right) \rightarrow L_{2}^{-1}\left(\mathbb{R}_{+}\right)$ defined by $\mathcal{L} u=-u^{\prime \prime}+q u$. To be more precise, Maz'ya and Verbitsky studied in $[\mathrm{MV}]$ the problem of identifying potentials $q$ for which the inequality

$$
\left.\left.\left|\int_{\mathbb{R}_{+}}\right| u(t)\right|^{2} q(t) d t\left|\leq \mathrm{const} \int_{\mathbb{R}_{+}}\right| u^{\prime}(t)\right|^{2} d t
$$

holds for any $C^{\infty}$ compactly supported function $u$ on $(0, \infty)$. This inequality is in turn equivalent to the boundedness of the quadratic form

$$
\left|\int_{\mathbb{R}_{+}} u(t) \overline{v(t)} q(t) d t\right| \leq \mathrm{const}\left\|u^{\prime}\right\|_{L^{2}\left(\mathbb{R}_{+}\right)}\left\|v^{\prime}\right\|_{L^{2}\left(\mathbb{R}_{+}\right)} .
$$

In $[\mathrm{MV}]$ under the assumption that the limit

$$
\lim _{y \rightarrow \infty} \int_{x}^{y} q(t) d t=\int_{x}^{\infty} q(t) d t \stackrel{\text { def }}{=} \varphi(x)
$$

exists for any $x>0$ the problem of boundedness (compactness) of the quadratic form (1.10) was reduced to the problem of boundedness (compactness) of the operator $Q_{\varphi}$ on $L^{2}\left(\mathbb{R}_{+}\right)$. Note that in [MV] the authors also obtained boundedness and compactness criteria for the operators $Q_{\varphi}$ in terms of conditions (1.7) and (1.9).

We also mention here the papers $[\mathrm{OP}]$ and $[\mathrm{AO}]$ where the authors study the properties of the imbedding operators from $\stackrel{\circ}{L}_{p}^{l}(\Omega)$ to $L^{p}(\Omega)$, where $\Omega$ is a domain in $\mathbb{R}^{n}$. There results also lead to certain estimates of $s_{n}\left(Q_{\varphi}^{+}\right)$for $\varphi$ supported on $[0, a], a \in \mathbb{R}_{+}$.

The conditions in the above definition are conditions on the size of $\varphi$ only, and define Banach lattices of functions on $\mathbb{R}_{+}$. Thus, if $|\psi| \leq|\varphi|$ and $Q_{\varphi}$ is bounded, compact, or belongs to $\boldsymbol{S}_{p}, p>1$, then $Q_{\psi}$ has the same property and, for example, $\left\|Q_{\psi}\right\|_{\boldsymbol{S}_{p}} \leq C_{p}\left\|Q_{\varphi}\right\|_{\boldsymbol{S}_{p}}$. Moreover, we will see that the same conditions are necessary and sufficient for these properties for the operators $Q_{\varphi}^{+}$and $Q_{\varphi}^{-}$too; thus, if one of the three operators has one these properties, then all three have it. These results for $Q_{\varphi}^{+}$are not new, see for example [ES, EEH, No, NeS, St], and the results for $Q_{\varphi}$ can easily be derived. Nevertheless we give complete proofs, by another method, as a background for the case $p \leq 1$.

At $p=1$, there is a kind of threshold, and for $\boldsymbol{S}_{p}, p \leq 1$, the situation is much more complex. First, $Q_{\varphi}^{+}$and $Q_{\varphi}^{-}$never belong to $\boldsymbol{S}_{1}$, except in the trivial case $\varphi=0$ a.e., when the operators vanish (Theorem 6.6). Secondly, although $\varphi \in X_{1}$ is a necessary condition for $Q_{\varphi} \in \boldsymbol{S}_{1}$, it is not sufficient. Indeed, for $p \leq 1$, we 
have not succeeded in finding both necessary and sufficient conditions for $Q_{\varphi} \in \boldsymbol{S}_{p}$, and any such conditions would have to be fairly complicated. For one thing, the property $Q_{\varphi} \in \boldsymbol{S}_{p}$ does not depend on the size of $\varphi$ only; although $\varphi_{0}(x)=\chi_{[0,1]}$, the characteristic function of the unit interval, yields an operator $Q_{\varphi_{0}}$ of rank 1, we show (see the example following Theorem 6.5) that there exists a function $\psi$ with $|\psi|=\left|\varphi_{0}\right|$ such that $Q_{\psi} \notin \boldsymbol{S}_{1}$. In the positive direction we show ( $\$ 5$ and $\S 10)$ that if $\varphi$ is sufficiently smooth and decays sufficiently rapidly at infinity, then $Q_{\varphi} \in \boldsymbol{S}_{p}, 1 / 2<p \leq 1$. Conversely, we give in $\S 15$ (Theorem 15.22) a necessary condition on the $L^{1}$ modulus of continuity for $Q_{\varphi} \in \boldsymbol{S}_{1}$.

At $p=1 / 2$ there is a second threshold. We prove in $\S 8$ and $\S 9$, by two different methods, that if $\varphi$ is smooth (locally absolutely continuous is enough), then $Q_{\varphi}$ never belongs to $\boldsymbol{S}_{1 / 2}$ except when $\varphi=0$ a.e. More precisely, if $\varphi$ is sufficiently smooth and decays sufficiently rapidly at infinity, and does not vanish identically, then the singular numbers $s_{n}\left(Q_{\varphi}\right)$ decay asymptotically exactly like $n^{-2}$ (Theorem 9.3).

On the other hand, $Q_{\varphi}$ may belong to $\boldsymbol{S}_{1 / 2}$ for non-smooth functions: It is easily seen that if $\varphi$ is a step function, then $Q_{\varphi}$ has finite rank, and thus $Q_{\varphi} \in \boldsymbol{S}_{p}$ for every $p>0$. Taking suitable infinite sums of step functions we find also other functions in $\boldsymbol{S}_{p}, p \leq 1 / 2$.

The role of smoothness is thus complicated and not well understood. It seems to be a help towards $Q_{\varphi} \in \boldsymbol{S}_{p}$ for $1 / 2<p \leq 1$, but it is not necessary and it completely prevents $Q_{\varphi} \in \boldsymbol{S}_{p}$ for $p \leq 1 / 2$. On the other hand, it is irrelevant for $p>1$.

As said above, $Q_{\varphi}$ has finite rank when $\varphi$ is a step function. We show in Theorem 12.2 that this is the only case when $Q_{\varphi}$ has finite rank.

The kernel in Definition (1.1) is symmetric, and thus $Q_{\varphi}$ is self-adjoint if and only if $\varphi$ is real. In $\S 4$ we show that $Q_{\varphi}$ is a positive operator if and only if $\varphi$ is a nonnegative non-increasing function. In this special case, for each $p>1 / 2, Q_{\varphi} \in \boldsymbol{S}_{p}$ if and only if $\varphi \in X_{p}$. In this case we also give an even simpler necessary and sufficient conditions for boundedness, compactness and $Q_{\varphi} \in \boldsymbol{S}_{p}, p>1 / 2$ (Theorem 4.6). In particular, for positive operators we have a necessary and sufficient condition for $p=1$ too.

When $\varphi$ is real and thus $Q_{\varphi}$ is self-adjoint, the singular values are the absolute values of the eigenvalues. In $\S 9$, we study the eigenvalues, which leads to a SturmLiouville problem that we study. We include one example (see §9), where the singular values can be calculated exactly by this method. We give also another example (Theorem 6.5) where the singular values are calculated within a constant factor by Fourier analysis.

In $\S 13$ and $\S 14$, we consider related families of operators acting on $L^{2}(\mathbb{R})$ and $\ell^{2}(\mathbb{Z})$; the latter operators include some given by weighted Hankel matrices. 
We further study operations acting on bounded linear operators on $L^{2}\left(\mathbb{R}_{+}\right)$related to the class of operators $Q_{\varphi}$. We study Schur multipliers given by functions of the form $\varphi(\max \{x, y\})$ in $\S 7$ and properties of the averaging projection onto the operators of the form $Q_{\varphi}$ in $\S 11$.

We give in this paper several necessary conditions and sufficient conditions for properties such as $Q_{\varphi} \in \boldsymbol{S}_{p}$. In all cases there are corresponding norm estimates, which follow by inspection of the proofs or by the closed graph theorem, although we usually do not state these estimates explicitly.

We denote by $|I|$ the length of an interval $I$. We also use $|S|$ for the cardinality of a finite set $S$; there is no danger of confusion.

We use $c$ and $C$, sometimes with subscripts or superscripts, to denote various unspecified constants, not necessarily the same on different occurrences. These constants are universal unless we indicate otherwise by subscripts.

\section{Preliminaries}

Definition (1.1) shows that the adjoint $Q_{\varphi}^{*}=Q_{\bar{\varphi}}$; in particular, $Q_{\varphi}$ is self-adjoint if and only if $\varphi$ is real. Similarly, $\left(Q_{\varphi}^{+}\right)^{*}=Q_{\bar{\varphi}}^{-}$; which has the same norm and singular numbers as $Q_{\varphi}^{-}$. Hence, we will mainly consider $Q_{\varphi}^{+}$; all results obtained in this paper for $Q_{\varphi}^{+}$immediately holds for $Q_{\varphi}^{-}$too.

Schatten classes. We denote the singular numbers of a bounded operator $T$ on a Hilbert space (or from one Hilbert space into another) by $s_{n}(T), n=0,1,2, \ldots$; thus $s_{n}(T) \stackrel{\text { def }}{=} \inf \{\|T-R\|: \operatorname{rank}(R) \leq n\}$. We will frequently use the simple facts

$$
s_{m+n}(T+R) \leq s_{m}(T)+s_{n}(R), \quad m, n \geq 0,
$$

and

$$
s_{m+n}(T R) \leq s_{m}(T) s_{n}(R), \quad m, n \geq 0,
$$

Recall that the Schatten-von Neumann classes $\boldsymbol{S}_{p}, 0<p<\infty$ are defined by

$$
\boldsymbol{S}_{p}=\left\{T: \sum_{n \geq 0} s_{n}(T)^{p}<\infty\right\},
$$

and the Schatten-Lorentz classes $\boldsymbol{S}_{p, q}$ are defined by

$$
\begin{aligned}
& \boldsymbol{S}_{p, q}=\left\{T: \sum_{n \geq 0}\left(s_{n}(T)\right)^{q}(1+n)^{q / p-1}<\infty\right\}, \quad 0<p<\infty, 0<q<\infty, \\
& \boldsymbol{S}_{p, \infty}=\left\{T: s_{n}(T) \leq \operatorname{const}(1+n)^{-1 / p}\right\}, \quad 0<p<\infty .
\end{aligned}
$$


See for example [GK1] and [BS].

Other intervals. We have defined our operators for the interval $\mathbb{R}_{+}=(0, \infty)$. More generally, for any interval $I \subseteq \mathbb{R}$ and a function $\varphi \in L_{\text {loc }}^{1}(I)$, we define $Q_{\varphi}^{I}$ to be the integral operator on $L^{2}(I)$ with kernel $\varphi(\max (x, y))$.

It is easily seen that if $I=(-\infty, a)$ with $-\infty<a \leq \infty$, then $Q_{\varphi}^{I}$ is bounded only for $\varphi=0$ a.e. By translation invariance, it remains only to consider the cases $I=(0, \infty)$, as above, and $I=(0, a)$ for some finite $a$. The latter case will be used sometimes below, but it can always be reduced to the case $(0, \infty)$. Indeed, if we extend $\varphi$ to $(0, \infty)$ letting $\varphi=0$ on $(a, \infty)$, then $Q_{\varphi}^{I}$ and $Q_{\varphi}$ may be identified. (Formally, they are defined on different spaces, and $Q_{\varphi}^{I}$ is the restriction of $Q_{\varphi}$ to $L^{2}(I)$, but the complementary restriction to $L^{2}(a, \infty)$ vanishes. In particular, $Q_{\varphi}^{I}$ and $Q_{\varphi}$ have the same singular numbers.)

The case of a finite interval can also be reduced to $[0,1]$ by the following simple homogeneity result.

Lemma 2.1. If $t>0$, and $\varphi_{t}(x)=t \varphi(t x)$, then $Q_{\varphi}$ and $Q_{\varphi_{t}}$ are unitarily equivalent. Similarly, for a subinterval $I \subset(0, \infty), Q_{\varphi}^{I}$ and $Q_{\varphi_{t}}^{t^{-1} I}$ are unitarily equivalent.

Proof. The mapping $T_{t}: f(x) \mapsto t^{1 / 2} f(t x)$ is a unitary operator in $L^{2}\left(\mathbb{R}_{+}\right)$, and $Q_{\varphi_{t}}=T_{t} Q_{\varphi} T_{t}^{-1}$.

Note that the spaces $X_{p}$ have the homogeneity property exhibited in this lemma: if $\varphi \in X_{p}$, then $\varphi_{t} \in X_{p}$ with the same norm. Of course, it is natural to have this property for any necessary or sufficient condition for $Q_{\varphi} \in \boldsymbol{S}_{p}$.

Distributions. We can also define the operators $Q_{\varphi}, Q_{\varphi}^{+}$, and $Q_{\varphi}^{-}$in the case when $\varphi$ is a distribution.

For an open subset $G$ of $\mathbb{R}^{n}$ we denote by $\mathcal{D}(G)$ the space of compactly supported $C^{\infty}$ functions in $G$ and denote by $\mathcal{D}^{\prime}(G)$ the space of distributions on $G$, i.e., continuous linear functionals on $\mathcal{D}(G)$. We refer the reader to [Sch] for basic facts about distributions. We use the notation $\langle\varphi, f\rangle$ for $\varphi(f)$, where $\varphi \in \mathcal{D}^{\prime}(G)$ and $f \in \mathcal{D}(G)$.

Suppose now that $\Sigma$ and $\Omega$ are open subsets of $\mathbb{R}$. In this paper we usually consider the case when $\Sigma=\Omega=\mathbb{R}_{+}$or $\Sigma=\Omega=\mathbb{R}$. Let $\Phi \in \mathcal{D}^{\prime}(\Sigma \times \Omega)$. We say that $\Phi$ determines a bounded linear operator from $L^{2}(\Omega)$ into $L^{2}(\Sigma)$ if there exists a constant $C$ such that $|\langle\Phi(x, y), f(y) \overline{g(x)}\rangle| \leq C\|f\|_{L^{2}}\|g\|_{L^{2}}$ for any $f \in \mathcal{D}(\Omega)$ and any $g \in \mathcal{D}(\Sigma)$; the corresponding operator $T$ then is given by $\langle T f, g\rangle=\langle\Phi(x, y), f(y) \overline{g(x)}\rangle$ and $\Phi$ is called the kernel of $T$. We say that $\Phi$ determines an operator in $\boldsymbol{S}_{p}$ if this operator is an operator from $L^{2}(\Omega)$ into $L^{2}(\Sigma)$ of class $\boldsymbol{S}_{p}$. Note that for any bounded operator $T: L^{2}(\Omega) \rightarrow L^{2}(\Sigma)$ there exists a distribution $\Phi \in \mathcal{D}^{\prime}(\Sigma \times \Omega)$ which determines the operator $T$ (a special case of Schwartz's kernel theorem). Indeed, it is easy to see that any function in $\mathcal{D}(\Omega \times \Sigma)$ 
defines an operator from $L^{2}(\Sigma)$ into $L^{2}(\Omega)$ of class $\boldsymbol{S}_{1}$, and we have a continuous imbedding $j: \mathcal{D}(\Omega \times \Sigma) \rightarrow \boldsymbol{S}_{1}\left(L^{2}(\Sigma), L^{2}(\Omega)\right)$. We may define the distribution $\Phi_{T} \in \mathcal{D}^{\prime}(\Sigma \times \Omega)$ by the following formula $\left\langle\Phi_{T}(x, y), f(x, y)\right\rangle \stackrel{\text { def }}{=} \operatorname{trace}(T A)$, where $f \in \mathcal{D}(\Sigma \times \Omega)$ and $A$ is the integral operator with kernel function $f(y, x)$. Clearly, $\Phi_{T}$ determines the operator $T$.

We also consider the space $\mathcal{S}\left(\mathbb{R}^{n}\right)$ of infinitely smooth functions whose derivatives of arbitrary orders decay at infinity faster than $(1+|x|)^{-n}$ for any $n \in \mathbb{Z}_{+}$and the dual space $\mathcal{S}^{\prime}\left(\mathbb{R}^{n}\right)$ of tempered distributions (see [Sch] for basic facts). Recall that the Fourier transform

$$
f \mapsto(\mathcal{F} f)(x) \stackrel{\text { def }}{=} \int_{\mathbb{R}^{n}} f(t) e^{-2 \pi \mathrm{i}(t, x)} d t
$$

where $(t, x)$ is the scalar product of $x$ and $t$ in $\mathbb{R}^{n}$, is an isomorphism of $\mathcal{S}\left(\mathbb{R}^{n}\right)$ onto itself, and that it can be extended to $\mathcal{S}^{\prime}\left(\mathbb{R}^{n}\right)$ by duality.

We need the following elementary facts.

Lemma 2.2. Suppose that a distribution $\Phi \in \mathcal{D}^{\prime}\left(\mathbb{R}^{2}\right)$ determines a bounded operator on $L^{2}(\mathbb{R})$. Then $\Phi$ is a tempered distribution.

Proof. It is easy to see that any function $\Phi \in \mathcal{S}\left(\mathbb{R}^{2}\right)$ determines an operator of class $\boldsymbol{S}_{1}$ and that the corresponding imbedding of $\mathcal{S}\left(\mathbb{R}^{2}\right)$ into $\boldsymbol{S}_{1}$ is continuous. The result follows now by duality.

Lemma 2.3. Let $\Phi \in \mathcal{S}^{\prime}\left(\mathbb{R}^{2}\right)$. Consider the distribution $\Psi$ on $\mathbb{R}^{2}$ defined by $\Psi(x, y) \stackrel{\text { def }}{=}(\mathcal{F} \Phi)(x,-y)$. Then $\Phi$ determines a bounded operator on $L^{2}(\mathbb{R})$ if and only if $\Psi$ does. Moreover, these two operators are unitarily equivalent.

Proof. It suffices to observe that

$$
\begin{aligned}
\langle\Psi(x, y), f(y) \overline{g(x)}\rangle & =\langle\Phi(x, y), \mathcal{F}(f(-y) \overline{g(x)})\rangle \\
& =\langle\Phi(x, y),(\mathcal{F} f)(-y) \overline{\mathcal{F} g(-x)})\rangle
\end{aligned}
$$

Now we are ready to define the operators $Q_{\varphi}, Q_{\varphi}^{+}$and $Q_{\varphi}^{-}$in the case where $\varphi$ is distribution.

It is not hard to see that the operator $f \mapsto \int_{0}^{x} f(x, y) d y$ is a continuous operator from $\mathcal{D}\left(\mathbb{R}_{+} \times \mathbb{R}_{+}\right)$into $\mathcal{D}\left(\mathbb{R}_{+}\right)$. Hence, with any $\varphi \in \mathcal{D}^{\prime}\left(\mathbb{R}_{+}\right)$we can associate the 
distributions $\Lambda_{\varphi}^{+}, \Lambda_{\varphi}^{-}$, and $\Lambda_{\varphi}$ in $\mathcal{D}^{\prime}\left(\mathbb{R}_{+} \times \mathbb{R}_{+}\right)$defined by

$$
\begin{aligned}
& \left\langle\Lambda_{\varphi}^{+}, f(x, y)\right\rangle \stackrel{\text { def }}{=}\left\langle\varphi, \int_{0}^{x} f(x, y) d y\right\rangle, \\
& \left\langle\Lambda_{\varphi}^{-}, f(x, y)\right\rangle \stackrel{\text { def }}{=}\left\langle\varphi, \int_{0}^{x} f(y, x) d y\right\rangle
\end{aligned}
$$

and

$$
\Lambda_{\varphi} \stackrel{\text { def }}{=} \Lambda_{\varphi}^{+}+\Lambda_{\varphi}^{-}
$$

For a distribution $\varphi$ in $\mathcal{D}^{\prime}\left(\mathbb{R}_{+}\right)$, we can consider now the operators $Q_{\varphi}^{+}, Q_{\varphi}^{-}$, and $Q_{\varphi}$ determined by the distributions $\Lambda_{\varphi}^{+}, \Lambda_{\varphi}^{-}$, and $\Lambda_{\varphi}$ respectively. It is easy to see that in case $\varphi \in L_{\text {loc }}^{1}\left(\mathbb{R}_{+}\right)$, the new definition coincides with the old one. The following theorem shows however that if one of those operators is bounded on $L^{2}\left(\mathbb{R}_{+}\right)$, then $\varphi$ must be a locally integrable function on $\mathbb{R}_{+}$, and so we have not enlarged the class of bounded operators of the form $Q_{\varphi}$.

Theorem 2.4. Let $\varphi \in \mathcal{D}^{\prime}\left(\mathbb{R}_{+}\right)$. Suppose that at least one of the distributions $\Lambda_{\varphi}^{+}, \Lambda_{\varphi}^{-}$, or $\Lambda_{\varphi}$ determines a bounded operator on $L^{2}\left(\mathbb{R}_{+}\right)$. Then $\varphi \in L_{\mathrm{loc}}^{2}\left(\mathbb{R}_{+}\right)$.

Proof. We consider the cases of the distributions $\Lambda_{\varphi}^{+}$and $\Lambda_{\varphi}$. For $\Lambda_{\varphi}^{-}$the proof is the same as for $\Lambda_{\varphi}^{+}$. Let $a \in \mathbb{R}_{+}$. Fix a function $f_{0} \in \mathcal{D}\left(\mathbb{R}_{+}\right)$such that $\operatorname{supp} f_{0} \subset[0, a]$ and $\int_{\mathbb{R}_{+}} f_{0}(x) d x=1$. We have

$$
\left\langle\Lambda_{\varphi}^{+}, f_{0}(y) \overline{g(x)}\right\rangle=\left\langle\Lambda_{\varphi}, f_{0}(y) \overline{g(x)}\right\rangle=\langle\varphi, \bar{g}\rangle
$$

for any $g \in \mathcal{D}\left(\mathbb{R}_{+}\right)$with supp $g \subset[a,+\infty)$. Therefore

$$
|\langle\varphi, \bar{g}\rangle| \leq C\left\|f_{0}\right\|_{L^{2}\left(\mathbb{R}_{+}\right)}\|g\|_{L^{2}\left(\mathbb{R}_{+}\right)}
$$

for any $g \in \mathcal{D}(a, \infty)$. Thus, $\varphi \mid(a, \infty) \in L^{2}(a, \infty)$.

Triangular projection. On the class of operators on $\boldsymbol{S}_{p}\left(L^{2}\left(\mathbb{R}_{+}\right)\right), p<\infty$, we define the operator of triangular projection $\mathcal{P}$ as follows. Consider first the case $p \leq 2$. Let $T$ be an operator on $L^{2}\left(\mathbb{R}_{+}\right)$of class $\boldsymbol{S}_{p}, p \leq 2$. Then $T$ is an integral operator with kernel function $k_{T}$ :

$$
(T f)(x)=\int_{0}^{\infty} k_{T}(x, y) f(y) d y, \quad f \in L^{2}\left(\mathbb{R}_{+}\right) .
$$

Then by definition

$$
(\mathcal{P} T f)(x)=\int_{0}^{x} k_{T}(x, y) f(y) d y, \quad f \in L^{2}\left(\mathbb{R}_{+}\right) .
$$


It is well known that

$$
\|\mathcal{P} T\|_{\boldsymbol{S}_{p}} \leq c_{p}\|T\|_{\boldsymbol{S}_{p}}, \quad 1<p \leq 2,
$$

where $c_{p}$ depends only on $p$. This allows one to extend by duality the definition of $\mathcal{P}$ and inequality (2.8) to the case $2 \leq p<\infty$. Note also that $\mathcal{P}$ has weak type $(1,1)$, i.e.,

$$
s_{n}(\mathcal{P} T) \leq \operatorname{const}(1+n)^{-1}\|T\|_{S_{1}}, \quad T \in \boldsymbol{S}_{1} .
$$

We will need these results on the triangular projection $\mathcal{P}$ in a more general situation. Let $\mu$ and $\nu$ be regular Borel measures on $\mathbb{R}_{+}$. As above we can associate with any operator $T$ from $L^{2}(\mu)$ to $L^{2}(\nu)$ of class $\boldsymbol{S}_{2}$ the operator $\mathcal{P} T$ by multiplying the kernel function of $T$ by the characteristic function of the set $\left\{(x, y) \in \mathbb{R}_{+}^{2}: 0<y<x\right\}$.

Theorem 2.5. $\mathcal{P}$ is a bounded linear projection on $\boldsymbol{S}_{p}\left(L^{2}(\mu), L^{2}(\nu)\right)$ for $1<p<\infty$ and $\mathcal{P}$ has weak type $(1,1)$, i.e., $\mathcal{P}$ is a bounded linear operator from $\boldsymbol{S}_{1}\left(L^{2}(\mu), L^{2}(\nu)\right)$ to $\boldsymbol{S}_{1, \infty}\left(L^{2}(\mu), L^{2}(\nu)\right)$.

Theorem 2.5 is well known at least when $\mu=\nu$. Let us explain how to reduce Theorem 2.5 to the case of the triangular projection onto the upper triangular matrices.

Let $\left\{\mathcal{K}_{j}\right\}_{j \geq 0}$ and $\left\{\mathcal{H}_{k}\right\}_{k \geq 0}$ be Hilbert spaces. Put $\mathcal{K} \stackrel{\text { def }}{=} \bigoplus_{j \geq 0} \mathcal{K}_{j}$ and $\mathcal{H} \stackrel{\text { def }}{=} \bigoplus_{k \geq 0} \mathcal{H}_{k}$. We identify operators $A \in \mathcal{B}(\mathcal{H}, \mathcal{K})$ with its block matrix representation $\left\{A_{j k}\right\}_{j, k \geq 0}$, where $A_{j k} \in \mathcal{B}\left(\mathcal{H}_{k}, \mathcal{K}_{j}\right)$. We define the triangular projection $\mathcal{P}$ by $(\mathcal{P} A)_{j k} \stackrel{\text { def }}{=} A_{j k}$ for $j>k$ and $(\mathcal{P} A)_{j k} \stackrel{\text { def }}{=} 0$ for $j \leq k$.

Lemma 2.6. Let $1<p<\infty$. Then $\mathcal{P}$ is bounded on $\boldsymbol{S}_{p}(\mathcal{H}, \mathcal{K})$ and has weak type $(1,1)$. Moreover, the norms of $\mathcal{P}$ can be bounded independently of $\mathcal{H}$ and $\mathcal{K}$.

In the case $\operatorname{dim} \mathcal{K}_{j}=\operatorname{dim} \mathcal{H}_{k}=1$, this is the Krein-Matsaev theorem (it is equivalent to Theorems III.2.4 and III.6.2 of [GK2], see also Theorem IV.8.2 of [GK1]). In general the result can be reduced easily to this special case. Indeed, it is easy to reduce the general case to the case when $\operatorname{dim} \mathcal{H}_{j}=\operatorname{dim} \mathcal{K}_{j}<\infty$. Then it is easy to see that if $A \in \boldsymbol{S}_{p}, 1 \leq p<\infty$, then the diagonal part of $A$ also belongs to $\boldsymbol{S}_{p}$, and so we may assume without loss of generality that $A_{j j}=0, j \in \mathbb{Z}_{+}$. We can take an orthonormal basis in each $\mathcal{H}_{j}$ and consider the orthonormal basis of $\mathcal{H}$ that consists of those basis vectors of $\mathcal{H}_{j}, j \in \mathbb{Z}_{+}$. Then we can consider the matrix representation of $A$ with respect to this orthonormal basis. We have now two triangular projections: with respect to the orthonormal basis and the projection $\mathcal{P}$, the triangular with respect to the the decomposition $\mathcal{H}=\bigoplus_{k \geq 0} \mathcal{H}_{k}$. It is not hard to check that since the diagonal part of $A$ is zero, both triangular 
projections applied to $A$ give the same result. This reduces the general case to the Krein-Matsaev theorem mentioned above.

Now it is easy to deduce Theorem 2.5 from Lemma 2.6.

Proof of Theorem 2.5. Let $T$ be an integral operator with kernel function $k$. For $\varepsilon>0$ we put $k_{\varepsilon}(x, y) \stackrel{\text { def }}{=} k(x, y) \chi_{\left\{(x, y) \in \mathbb{R}_{+}^{2}:\left[\frac{x}{\varepsilon}\right] \varepsilon>y>0\right\}}$, where $[a]$ denote the largest integer that is less than or equal to $a$. Suppose that $p>1$. It is sufficient to consider the case $1<p \leq 2$ and then use duality. Let $T_{\varepsilon}$ be the integral operator with kernel function $k_{\varepsilon}$. It follows easily from Lemma 2.6 that

$$
\left\|T_{\varepsilon}\right\|_{\boldsymbol{S}_{p}\left(L^{2}(\mu), L^{2}(\nu)\right)} \leq c_{p}\|T\|_{\boldsymbol{S}_{p}\left(L^{2}(\mu), L^{2}(\nu)\right)}
$$

for any $\varepsilon>0$. Clearly, $T_{\varepsilon} \rightarrow T$ in the weak operator topology as $\varepsilon \rightarrow 0$. It follows that $\|\mathcal{P} T\|_{\boldsymbol{S}_{p}\left(L^{2}(\mu), L^{2}(\nu)\right)} \leq c_{p}\|T\|_{\boldsymbol{S}_{p}\left(L^{2}(\mu), L^{2}(\nu)\right)}$. The case $p=1$ may be considered in the same way.

We have $Q_{\varphi}^{+}=\mathcal{P} Q_{\varphi}$, which together with the equivalence

$$
Q_{\varphi}^{+} \in \boldsymbol{S}_{p} \Longleftrightarrow Q_{\varphi}^{-} \in \boldsymbol{S}_{p}
$$

yields

$$
Q_{\varphi}^{+} \in \boldsymbol{S}_{p} \Longleftrightarrow Q_{\varphi} \in \boldsymbol{S}_{p}
$$

for $1<p<\infty$. We will give a direct proof of this in Theorem 3.3.

We introduce a more general operation. Let $A$ be a measurable subset of $\mathbb{R}_{+} \times \mathbb{R}_{+}$. For an operator $T$ on $L^{2}\left(\mathbb{R}_{+}\right)$of class $\boldsymbol{S}_{2}$ with kernel function $k_{T}$ we consider the integral operator $\mathcal{P}_{A} T$ whose kernel function is $\chi_{A} k_{T}$, where $\chi_{A}$ is the characteristic function of $A$. In other words,

$$
\left(\mathcal{P}_{A} T f\right)(x)=\int_{0}^{\infty} \chi_{A}(x, y) k_{T}(x, y) f(y) d y .
$$

If $0<p \leq 2$ and $\mathcal{P}_{A}$ maps $\boldsymbol{S}_{p}$ into itself, it follows from the closed graph theorem that the linear transformation $\mathcal{P}_{A}$ is a bounded linear operator on $\boldsymbol{S}_{p}$. If $1<p \leq 2$ and $\mathcal{P}_{A}$ is a bounded linear operator on $\boldsymbol{S}_{p}$, then by duality we can define in a natural way the bounded linear operator $\mathcal{P}_{A}$ on $\boldsymbol{S}_{p^{\prime}}$. If $\mathcal{P}_{A}$ is bounded on $\boldsymbol{S}_{1}$, we can define by duality $\mathcal{P}_{A}$ on the space $\mathcal{B}\left(L^{2}\left(\mathbb{R}_{+}\right)\right)$of bounded linear operators on $L^{2}\left(\mathbb{R}_{+}\right)$. Note that the projection $\mathcal{P}$ defined by $(2.7)$ is equal to $\mathcal{P}_{A}$ with $A=\left\{(x, y): x \in \mathbb{R}_{+}, y \leq x\right\}$.

\section{Boundedness, compactness, and $p>1$}

Recall the spaces $X_{p}$ defined in the Introduction.

Theorem 3.1. Let $\varphi \in L_{\text {loc }}^{2}\left(\mathbb{R}_{+}\right)$. The following are equivalent: 
(i) $Q_{\varphi}$ is bounded on $L^{2}\left(\mathbb{R}_{+}\right)$;

(ii) $Q_{\varphi}^{+}$is bounded on $L^{2}\left(\mathbb{R}_{+}\right)$;

(iii) $\varphi \in X_{\infty}$.

Recall that the equivalence of (i) and (iii) was also established in [MV] by a different method.

Proof. Let us show that (ii) implies (i). If $Q_{\varphi}^{+}$is bounded, then the integral operator $Q_{\varphi}^{-} \stackrel{\text { def }}{=} Q_{\varphi}-Q_{\varphi}^{+}$is also bounded, since its kernel function is the reflection of the kernel function of $Q_{\varphi}^{+}$with respect to the line $\{x=y\}$. Hence, $Q_{\varphi}$ is bounded.

Let us deduce now (iii) from (i). For $n \in \mathbb{Z}$ put

$$
A_{n}=\left[2^{n}, 2^{n+1}\right] \times\left[2^{n-1}, 2^{n}\right] .
$$

Certainly, if $Q_{\varphi}$ is bounded, then

$$
\sup _{n \in \mathbb{Z}}\left\|\mathcal{P}_{A_{n}} Q_{\varphi}\right\|<\infty .
$$

It is easy to see that $\mathcal{P}_{A_{n}} Q_{\varphi}$ is a rank one operator and

$$
\left\|\mathcal{P}_{A_{n}} Q_{\varphi}\right\|=\left(2^{n-1} \int_{2^{n}}^{2^{n+1}}|\varphi(x)|^{2} d x\right)^{1 / 2}
$$

which implies (1.6).

It remains to prove that (1.6) implies (ii). Put

$$
B=\bigcup_{n \in \mathbb{Z}} B_{n}
$$

where

$$
B_{n}=\left\{(x, y): 2^{n} \leq x \leq 2^{n+1}, 2^{n}<y<x\right\} .
$$

We also define the sets

$$
A_{n}^{(k)}=\left[2^{n}, 2^{n+1}\right] \times\left[2^{n-k}, 2^{n-k+1}\right]
$$

and

$$
A^{(k)}=\bigcup_{n \in \mathbb{Z}} A_{n}^{(k)}
$$

Clearly,

$$
\{(x, y): x>0,0<y<x\}=B \cup \bigcup_{k \geq 1} A^{(k)},
$$

and so

$$
\left\|Q_{\varphi}^{+}\right\| \leq\left\|\mathcal{P}_{B} Q_{\varphi}\right\|+\sum_{k \geq 1}\left\|\mathcal{P}_{A^{(k)}} Q_{\varphi}\right\| .
$$


Since the projections of the $B_{n}$ onto the coordinate axes are pairwise disjoint, it is straightforward to see that

$$
\left\|\mathcal{P}_{B} Q_{\varphi}^{+}\right\|=\sup _{n \in \mathbb{Z}}\left\|\mathcal{P}_{B_{n}} Q_{\varphi}^{+}\right\|
$$

Let $R_{n}$ be the integral operator with kernel function

$$
k_{R_{n}}(x, y)=\varphi(x) \chi_{\left[2^{n}, 2^{n+1}\right]}(x) \chi_{\left[2^{n}, 2^{n+1}\right]}(y) .
$$

Obviously, $\operatorname{rank} R_{n}=1$ and $\left\|R_{n}\right\|_{S_{2}}=\left\|R_{n}\right\|=\left(2^{n} \int_{2^{n}}^{2^{n+1}}|\varphi(x)|^{2} d x\right)^{1 / 2}$. It is also evident that $\mathcal{P} R_{n}=\mathcal{P}_{B_{n}} Q_{\varphi}^{+}$, and since $\mathcal{P}$ an orthogonal projection on $\boldsymbol{S}_{2}$, we have

$$
\begin{aligned}
\left\|\mathcal{P}_{B_{n}} Q_{\varphi}^{+}\right\| & =\left\|\mathcal{P} R_{n}\right\| \leq\left\|\mathcal{P} R_{n}\right\|_{S_{2}} \leq\left\|R_{n}\right\|_{S_{2}} \\
& =\left(2^{n} \int_{2^{n}}^{2^{n+1}}|\varphi(x)|^{2} d x\right)^{1 / 2} \leq \text { const }\|\varphi\|_{X_{\infty}} .
\end{aligned}
$$

Next, it is also easy to see that

$$
\left\|\mathcal{P}_{A^{(k)}} Q_{\varphi}\right\|=\sup _{n \in \mathbb{Z}}\left\|\mathcal{P}_{A_{n}^{(k)}} Q_{\varphi}\right\| .
$$

Also, $\mathcal{P}_{A_{n}^{(k)}} Q_{\varphi}$ has rank one and norm $\left(2^{n-k} \int_{2^{n}}^{2^{n+1}}|\varphi(x)|^{2} d x\right)^{1 / 2}$, and so

$$
\begin{aligned}
\sum_{k \geq 1}\left\|\mathcal{P}_{A^{(k)}} Q_{\varphi}\right\| & =\sum_{k \geq 1} \sup _{n \in \mathbb{Z}}\left(2^{n-k} \int_{2^{n}}^{2^{n+1}}|\varphi(x)|^{2} d x\right)^{1 / 2} \\
& =\sum_{k \geq 1} 2^{-k / 2} \sup _{n \in \mathbb{Z}}\left(2^{n} \int_{2^{n}}^{2^{n+1}}|\varphi(x)|^{2} d x\right)^{1 / 2} \\
& =\text { const } \sup _{n \in \mathbb{Z}}\left(2^{n} \int_{2^{n}}^{2^{n+1}}|\varphi(x)|^{2} d x\right)^{1 / 2} .
\end{aligned}
$$

The result follows now from (3.5).

Theorem 3.2. Let $\varphi \in L_{\mathrm{loc}}^{1}\left(\mathbb{R}_{+}\right)$. The following are equivalent:

(i) $Q_{\varphi}$ is compact on $L^{2}\left(\mathbb{R}_{+}\right)$;

(ii) $Q_{\varphi}^{+}$is compact on $L^{2}\left(\mathbb{R}_{+}\right)$;

(iii) $\varphi \in X_{\infty}^{0}$.

Recall that the equivalence of (i) and (iii) was also established in [MV] by a different method. 
Proof. It is easy to see that the estimates given in the proof of Theorem 3.1 actually lead to the proof of Theorem 3.2 ; for the step (i) $\Longrightarrow$ (iii) we observe that if $Q_{\varphi}$ is compact, then $\lim _{n \rightarrow \pm \infty}\left\|\mathcal{P}_{A_{n}} Q_{\varphi}\right\|=0$.

Theorem 3.3. Let $1<p<\infty$ and let $\varphi \in L_{\mathrm{loc}}^{1}\left(\mathbb{R}_{+}\right)$. Then the following conditions are equivalent:

(i) $Q_{\varphi} \in \boldsymbol{S}_{p}$;

(ii) $Q_{\varphi}^{+} \in \boldsymbol{S}_{p}$;

(iii) $\varphi \in X_{p}$.

Note that the fact that (ii) $\Leftrightarrow$ (iii) was proved in [No] by a different method, see also $[\mathrm{NeS}]$ and [St] for the case of more general Volterra operators.

Proof. The fact that (ii) $\Rightarrow$ (i) can be proved exactly as in the proof of Theorem 3.1. Let us show that (i) implies (iii). Consider the sets $A_{n}=A_{n}^{(1)}$ introduced in the proof of Theorem 3.1 (see (3.3)). Recall that $A^{(1)}=\bigcup_{n \in \mathbb{Z}} A_{n}^{(1)}$. It is easy to see that

Clearly,

$$
\left\|\mathcal{P}_{A^{(1)}} Q_{\varphi}\right\|_{\boldsymbol{S}_{p}} \leq\left\|Q_{\varphi}\right\|_{\boldsymbol{S}_{p}}
$$

$$
\left\|\mathcal{P}_{A^{(1)}} Q_{\varphi}\right\|_{\boldsymbol{S}_{p}}^{p}=\sum_{n \in \mathbb{Z}}\left\|\mathcal{P}_{A_{n}} Q_{\varphi}\right\|_{\boldsymbol{S}_{p}}^{p}
$$

the operator $\mathcal{P}_{A_{n}} Q_{\varphi}$ has rank one and

$$
\left\|\mathcal{P}_{A_{n}} Q_{\varphi}\right\|_{\boldsymbol{S}_{p}}=\left(2^{n-1} \int_{2^{n}}^{2^{n+1}}|\varphi(x)|^{2} d x\right)^{1 / 2}
$$

This implies (1.3).

Let us show that (1.3) implies (ii). Consider the sets, $B_{n}, B, A_{n}^{(k)}, A^{(k)}$ defined in (3.2), (3.1), (3.3), and (3.4). Clearly,

$$
\left\|Q_{\varphi}^{+}\right\|_{\boldsymbol{S}_{p}} \leq\left\|\mathcal{P}_{B} Q_{\varphi}^{+}\right\|_{\boldsymbol{S}_{p}}+\sum_{k \geq 1}\left\|\mathcal{P}_{A^{(k)}} Q_{\varphi}^{+}\right\|_{\boldsymbol{S}_{p}}
$$

Let us first estimate $\left\|\mathcal{P}_{B} Q_{\varphi}^{+}\right\|_{\boldsymbol{S}_{p}}$. Clearly,

$$
\left\|\mathcal{P}_{B} Q_{\varphi}^{+}\right\|_{\boldsymbol{S}_{p}}^{p}=\sum_{n \in \mathbb{Z}}\left\|\mathcal{P}_{B_{n}} Q_{\varphi}^{+}\right\|_{\boldsymbol{S}_{p}}^{p}
$$

Consider the rank one operators $R_{n}$ defined in (3.6). As in the proof of Theorem 3.1 we have $\mathcal{P} R_{n}=\mathcal{P}_{B_{n}} Q_{\varphi}^{+}$and since $\mathcal{P}$ is bounded on $\boldsymbol{S}_{p}$, we obtain

$$
\left\|\mathcal{P}_{B_{n}} Q_{\varphi}^{+}\right\|_{S_{p}}=\left\|\mathcal{P} R_{n}\right\|_{S_{p}} \leq \text { const }_{p}\left\|R_{n}\right\|_{S_{p}}=\operatorname{const}_{p}\left(2^{n} \int_{2^{n}}^{2^{n+1}}|\varphi(x)|^{2} d x\right)^{1 / 2}
$$


and so

$$
\left\|\mathcal{P}_{B} Q_{\varphi}^{+}\right\|_{\boldsymbol{S}_{p}}^{p} \leq \text { const }_{p} \sum_{n \in \mathbb{Z}}\left(2^{n} \int_{2^{n}}^{2^{n+1}}|\varphi(x)|^{2} d x\right)^{p / 2}
$$

It is also easy to see that

$$
\left\|\mathcal{P}_{A^{(k)}} Q_{\varphi}^{+}\right\|_{\boldsymbol{S}_{p}}^{p}=\sum_{n \in \mathbb{Z}}\left\|\mathcal{P}_{A_{n}^{(k)}} Q_{\varphi}^{+}\right\|_{\boldsymbol{S}_{p}}^{p}
$$

and, since $\mathcal{P}_{A_{n}^{(k)}} Q_{\varphi}$ has rank 1 ,

$$
\left\|\mathcal{P}_{A_{n}^{(k)}} Q_{\varphi}^{+}\right\|_{S_{p}}=\left\|\mathcal{P}_{A_{n}^{(k)}} Q_{\varphi}\right\|_{S_{p}}=\left(2^{n-k} \int_{2^{n}}^{2^{n+1}}|\varphi(x)|^{2} d x\right)^{1 / 2}
$$

and so

$$
\begin{aligned}
\left\|\mathcal{P}_{A^{(k)}} Q_{\varphi}^{+}\right\|_{\boldsymbol{S}_{p}} & =\left(\sum_{n \in \mathbb{Z}}\left(2^{n-k} \int_{2^{n}}^{2^{n+1}}|\varphi(x)|^{2} d x\right)^{p / 2}\right)^{1 / p} \\
& =2^{-k / 2}\left(\sum_{n \in \mathbb{Z}}\left(2^{n} \int_{2^{n}}^{2^{n+1}}|\varphi(x)|^{2} d x\right)^{p / 2}\right)^{1 / p}
\end{aligned}
$$

and

$$
\sum_{k \geq 1}\left\|\mathcal{P}_{A^{(k)}} Q_{\varphi}^{+}\right\|_{\boldsymbol{S}_{p}} \leq \mathrm{const}\left(\sum_{n \in \mathbb{Z}}\left(2^{n} \int_{2^{n}}^{2^{n+1}}|\varphi(x)|^{2} d x\right)^{p / 2}\right)^{1 / p}
$$

which completes the proof.

Remark. The same proof shows that for $p=1$, (ii) $\Rightarrow$ (i) $\Rightarrow$ (iii), but the final part of it fails because the triangular projection is not bounded on $\boldsymbol{S}_{1}$. We will see later that, indeed, none of the implications can be reversed for $p=1$.

In the Hilbert-Schmidt case $p=2$, the result simplifies further. Indeed, we have the equalities

$$
\left\|Q_{\varphi}\right\|_{\boldsymbol{S}_{2}}=\left(\int_{0}^{\infty} \int_{0}^{\infty}|\varphi(\max (x, y))|^{2} d x d y\right)^{1 / 2}=\left(2 \int_{0}^{\infty} x|\varphi(x)|^{2} d x\right)^{1 / 2}
$$

and

$$
\left\|Q_{\varphi}^{+}\right\|_{S_{2}}=2^{-1 / 2}\left\|Q_{\varphi}\right\|_{S_{2}}=\left\|x^{1 / 2} \varphi(x)\right\|_{2}=\|\varphi\|_{X_{2}}
$$




\section{Positive operators}

We consider the special case when $Q_{\varphi}$ is a positive operator, i.e., $\left\langle Q_{\varphi} f, f\right\rangle \geq 0$ for every $f \in L^{2}\left(\mathbb{R}_{+}\right)$. In this case we obtain rather complete results. We first characterize the corresponding symbols $\varphi$.

Theorem 4.1. Suppose that $\varphi \in L_{\mathrm{loc}}^{1}\left(\mathbb{R}_{+}\right)$is such that $Q_{\varphi}$ is a bounded operator. Then $Q_{\varphi}$ is a positive operator if and only if $\varphi$ is a.e. equal to a non-increasing, non-negative function.

Proof. Suppose that $Q_{\varphi}$ is positive. Define, for $z, h>0, f_{z, h}=h^{-1} \chi_{(z, z+h)}$ and let $\operatorname{Leb}(\varphi)$ be the set of Lebesgue points of $\varphi$. Then, if $z \in \operatorname{Leb}(\varphi)$,

$$
\begin{aligned}
\left|\left\langle Q_{\varphi} f_{z, h}, f_{z, h}\right\rangle-\varphi(z)\right| & =h^{-2}\left|\int_{z}^{z+h} \int_{z}^{z+h}(\varphi\{\max (x, y)\}-\varphi(z)) d x d y\right| \\
& \leq h^{-2} \int_{z}^{z+h} \int_{z}^{z+h}(|\varphi(x)-\varphi(z)|+|\varphi(y)-\varphi(z)|) d x d y \\
& =2 h^{-1} \int_{z}^{z+h}|\varphi(x)-\varphi(z)| d x \rightarrow 0
\end{aligned}
$$

as $h \rightarrow 0$. Since $\left\langle Q_{\varphi} f_{z, h}, f_{z, h}\right\rangle \geq 0$ for all $h>0$, this implies $\varphi(z) \geq 0$.

Moreover, if $z_{1}, z_{2} \in \operatorname{Leb}(\varphi)$ are two Lebesgue points with $z_{1}<z_{2}$ and $0<h<z_{2}-z_{1}$, then, similarly,

$$
\left\langle Q_{\varphi} f_{z_{1}, h}, f_{z_{2}, h}\right\rangle=\left\langle Q_{\varphi} f_{z_{2}, h}, f_{z_{1}, h}\right\rangle=h^{-1} \int_{z_{2}}^{z_{2}+h} \varphi(y) d y \rightarrow \varphi\left(z_{2}\right)
$$

as $h \rightarrow 0$, and thus, with $g_{h}=f_{z_{1}, h}-f_{z_{2}, h}$,

$$
\left\langle Q_{\varphi} g_{h}, g_{h}\right\rangle \rightarrow \varphi\left(z_{1}\right)+\varphi\left(z_{2}\right)-2 \varphi\left(z_{2}\right)=\varphi\left(z_{1}\right)-\varphi\left(z_{2}\right) .
$$

Hence, $\varphi\left(z_{1}\right) \geq \varphi\left(z_{2}\right)$.

It follows that the function $\tilde{\varphi}(x)=\sup \{\varphi(z): z \geq x, z \in \operatorname{Leb}(\varphi)\}$ is non-negative and non-increasing, and that $\varphi=\tilde{\varphi}$ a.e.

Conversely, if $\varphi$ is non-negative and non-increasing, then $\lim _{x \rightarrow \infty} \varphi(x)=0$, since a positive lower bound is impossible by Theorem 3.1. Thus there exists a measure $\mu$ on $(0, \infty)$ such that $\varphi(x)=\mu(x, \infty)$ a.e. If, say, $f$ is bounded with compact support in $(0, \infty)$, then by Fubini's theorem

$$
\begin{aligned}
\left\langle Q_{\varphi} f, f\right\rangle & =\iint \varphi(\max \{x, y\}) f(x) \bar{f}(y) d x d y=\iiint_{\max \{x, y\}<z} f(x) \bar{f}(y) d x d y d \mu(z) \\
& =\int_{0}^{\infty}\left|\int_{0}^{z} f(x) d x\right|^{2} d \mu \geq 0 .
\end{aligned}
$$


Hence, $Q_{\varphi}$ is a positive operator.

We have used the fact that $Q_{\varphi}$ is a sum of the Volterra operators $Q_{\varphi}^{+}$and $Q_{\varphi}^{-}$. Operators of the type $Q_{\varphi}$ also appear as the composition of Volterra operators.

Theorem 4.2. Suppose that $\psi_{1}$ and $\psi_{2}$ are functions on $\mathbb{R}_{+}$such that $Q_{\psi_{1}}$ and $Q_{\psi_{2}}$ are bounded linear operators. Let $\varphi$ be the function defined by

$$
\varphi(x)=\int_{x}^{\infty} \psi_{1}(t) \psi_{2}(t) d t .
$$

Then the operator $Q_{\varphi}$ is bounded and admits a factorization

$$
Q_{\varphi}=Q_{\psi_{1}}^{-} Q_{\psi_{2}}^{+}
$$

Proof. Let $k_{1}$ be the kernel function of $Q_{\psi_{1}}^{-}$and $k_{2}$ the kernel function of $Q_{\psi_{2}}^{+}$. We have

$$
k_{1}(x, t)= \begin{cases}\psi_{1}(t), & t \geq x \\ 0, & t<x\end{cases}
$$

and

$$
k_{2}(t, y)= \begin{cases}0, & t<y \\ \psi_{2}(t), & t \geq y\end{cases}
$$

Then the kernel function $k$ of the product $Q_{\psi_{1}}^{-} Q_{\psi_{2}}^{+}$is given by

$$
k(x, y)=\int_{\mathbb{R}_{+}} k_{1}(x, t) k_{2}(t, y) d t=\int_{\max \{x, y\}}^{\infty} \psi_{1}(t) \psi_{2}(t) d t=\varphi(\max \{x, y\})
$$

by the hypotheses; the integrals converge by Theorem 3.1 and the Cauchy-Schwarz inequality.

The function $\varphi$ in (4.1) is always locally absolutely continuous. In order to treat more general non-increasing $\varphi$, we define, for a positive measure $\mu$ on $(0, \infty)$, the operator $\mathbf{Q}_{\mu}^{+}: L^{2}(0, \infty) \rightarrow L^{2}(\mu)$ by $\mathbf{Q}_{\mu}^{+} f(x)=\int_{0}^{x} f(y) d y$. (Thus, the operator itself does not depend on $\mu$; only its range space does.) We have the following analogues of Theorems 3.1 and 3.3. (We leave the corresponding criterion for compactness to the reader.)

Theorem 4.3. Let $\mu$ be a positive measure on $\mathbb{R}_{+}$. The following are equivalent:

(i) $\mathrm{Q}_{\mu}^{+}$is bounded operator from $L^{2}\left(\mathbb{R}_{+}\right)$to $L^{2}(\mu)$;

(ii) $\sup _{n \in \mathbb{Z}} 2^{n} \mu\left[2^{n}, 2^{n+1}\right)<\infty$;

(iii) $\sup _{x>0} x \mu[x, \infty)<\infty$.

Theorem 4.4. Let $1<p<\infty$ and let $\mu$ be a positive measure on $\mathbb{R}_{+}$. The following conditions are equivalent: 
(i) $\mathbf{Q}_{\mu}^{+} \in \boldsymbol{S}_{p}$;

(ii) $\sum_{n \in \mathbb{Z}} 2^{n p / 2}\left(\mu\left[2^{n}, 2^{n+1}\right)\right)^{p / 2}<\infty$;

(iii) $\sum_{n \in \mathbb{Z}} 2^{n p / 2}\left(\mu\left[2^{n}, \infty\right)\right)^{p / 2}<\infty$;

(iv) $x^{1 / 2}(\mu(x, \infty))^{1 / 2} \in L^{p}(d x / x)$.

The proofs of Theorems 4.3 and 4.4 are almost the same as the proofs of Theorems 3.1 and 3.3. The main difference is that we have to apply the theorem on the boundedness of the triangular projection on $\boldsymbol{S}_{p}, 1<p<\infty$, in the case of weighted $L^{2}$ spaces (see Theorem 2.5).

Furthermore, the factorization in Theorem 4.2 extends.

Theorem 4.5. Suppose that $\mu$ is a positive measure on $\mathbb{R}_{+}$such that $\mathbf{Q}_{\mu}^{+}$is a bounded linear operator. Let $\varphi$ be the function defined by $\varphi(x)=\mu(x, \infty)$. Then the operator $Q_{\varphi}$ is bounded and $Q_{\varphi}=\left(\mathbf{Q}_{\mu}^{+}\right)^{*} \mathbf{Q}_{\mu}^{+}$.

Proof. By Theorem 4.3, $0 \leq \varphi(x) \leq C_{\varphi} / x$, and thus $Q_{\varphi}$ is bounded by Theorem 3.1.

If, say, $f, g \in L^{2}\left(\mathbb{R}_{+}\right)$are non-negative, then by Fubini's theorem

$$
\begin{aligned}
\left\langle\left(\mathbf{Q}_{\mu}^{+}\right)^{*} \mathbf{Q}_{\mu}^{+} f, g\right\rangle & =\left\langle\mathbf{Q}_{\mu}^{+} f, \mathbf{Q}_{\mu}^{+} g\right\rangle=\int_{0}^{\infty} \int_{0}^{z} f(x) d x \int_{0}^{z} \bar{g}(y) d y d \mu(z) \\
& =\iiint_{\max \{x, y\}<z} f(x) \bar{g}(y) d x d y d \mu(z)=\left\langle Q_{\varphi} f, g\right\rangle .
\end{aligned}
$$

For positive operators $Q_{\varphi}$, we have a simple result, Theorem 4.6 below. (For (i), cf. the discussion of the Hille condition in $[\mathrm{MV}]$.) $\mathbb{R}_{+}$.

Theorem 4.6. Suppose that $\varphi$ is a non-negative, non-increasing function on

(i) $Q_{\varphi}$ is bounded if and only if $x \varphi(x)$ is bounded.

(ii) $Q_{\varphi}$ is compact if and only if $x \varphi(x) \rightarrow 0$ as $x \rightarrow 0$ and as $x \rightarrow \infty$.

(iii) If $1 / 2<p<\infty$, then the following are equivalent:
(a) $Q_{\varphi} \in \boldsymbol{S}_{p}$;
(b) $\varphi \in X_{p}$;
(c) $x \varphi(x) \in L^{p}(d x / x)$.

Proof. The equivalence of $\varphi \in X_{p}$ and $x \varphi(x) \in L^{p}(d x / x)$ for non-increasing, non-negative $\varphi$ is elementary, using $\varphi\left(2^{n}\right)^{2} \geq 2^{-n} \int_{2^{n}}^{2^{n+1}}|\varphi|^{2} \geq \varphi\left(2^{n+1}\right)^{2}$. Hence, (i) follows from Theorem 3.1, and (iii) for $p>1$ from Theorem 3.3; furthermore, (ii) follows similarly from Theorem 3.2. 
For (iii) for a general $p>1 / 2$, we first note that any of the three conditions (a), (b) and (c) implies that $x \varphi(x)$ is bounded. (This follows by (i) for (a), and by elementary estimates for (b) and (c).) We can assume without loss of generality that $\varphi$ is right-continuous on $(0, \infty)$. If we let $\mu$ be the measure on $\mathbb{R}_{+}$with $\mu(x, \infty)=\varphi(x)$, then by Theorems 4.3 and $4.5, \mathrm{Q}_{\mu}^{+}$is bounded and $Q_{\varphi}=\left(\mathbf{Q}_{\mu}^{+}\right)^{*} \mathbf{Q}_{\mu}^{+}$. Hence, $Q_{\varphi} \in \boldsymbol{S}_{p} \Leftrightarrow \mathbf{Q}_{\mu}^{+} \in \boldsymbol{S}_{2 p}$, and the result follows by Theorem 4.4 .

We will see in the example given at the beginning of $\S 9$ that Theorem 4.6 (iii) does not extend to $p \leq 1 / 2$.

\section{A sufficient condition, $1 / 2<p \leq 1$}

By linearity, we immediately obtain from Theorem 4.6 a sufficient, but not necessary, condition for general symbols $\varphi$.

Definition. $Y_{p}$ is the subspace of $X_{p}$ spanned by non-increasing functions. I.e., $\varphi \in Y_{p}$ if and only if $\operatorname{Re} \varphi$ and $\operatorname{Im} \varphi$ both are differences of non-increasing functions in $X_{p}$.

Theorem 5.1. Let $p>1 / 2$. If $\varphi \in Y_{p}$, then $Q_{\varphi} \in \boldsymbol{S}_{p}$.

The condition $\varphi \in Y_{p}$ can be made more explicit and useful as follows. We denote by $\|\varphi\|_{B V(I)}^{\prime}$ the total variation of a function $\varphi$ over an interval $I$, and let $\|\varphi\|_{B V(I)} \stackrel{\text { def }}{=}\|\varphi\|_{B V(I)}^{\prime}+\sup _{I}|\varphi|$. Moreover, we let $V_{\varphi}(x)$ denote the total variation of a function $\varphi$ over the interval $[x, \infty)$. Note that if $\varphi$ is locally absolutely continuous, then $V_{\varphi}(x)=\int_{x}^{\infty}\left|\varphi^{\prime}(y)\right| d y$.

Lemma 5.2. Let $0<p<\infty$. If $\varphi$ is non-increasing, then

$$
\varphi \in Y_{p} \Leftrightarrow \varphi \in X_{p} \Leftrightarrow \int_{0}^{\infty}|x \varphi(x)|^{p} \frac{d x}{x}<\infty .
$$

Proof. For non-increasing $\varphi$, the first equivalence follows from the definition of $Y_{p}$, while the second equivalence was noted in the proof of Theorem 4.6.

Theorem 5.3. Let $\varphi$ be a function on $\mathbb{R}_{+}$and let $0<p<\infty$. The following are equivalent:

(i) $\varphi \in Y_{p}$;

(ii) $V_{\varphi} \in X_{p}$ and $\lim _{x \rightarrow \infty} \varphi(x)=0$;

(iii) $x V_{\varphi}(x) \in L^{p}(d x / x)$ and $\lim _{x \rightarrow \infty} \varphi(x)=0$; 
(iv) $\varphi$ has locally bounded variation, $\lim _{x \rightarrow \infty} \varphi(x)=0$ and $\sum_{n \in \mathbb{Z}} 2^{n p}\left(\int_{2^{n}}^{\infty}|d \varphi(x)|\right)^{p}<\infty ;$

(v) $\varphi$ has locally bounded variation, $\lim _{x \rightarrow \infty} \varphi(x)=0$ and

$$
\sum_{n \in \mathbb{Z}} 2^{n p}\left(\int_{2^{n}}^{2^{n+1}}|d \varphi(x)|\right)^{p}<\infty
$$

(vi) $\sum_{n \in \mathbb{Z}} 2^{n p}\|\varphi\|_{B V\left[2^{n}, 2^{n+1}\right]}^{p}<\infty$;

(vii) $\sum_{n \in \mathbb{Z}}\|x \varphi(x)\|_{B V\left[2^{n}, 2^{n+1}\right]}^{p}<\infty$.

Proof. To show that (i) implies (ii), it suffices to consider a non-increasing $\varphi \in X_{p}$; it is easily seen that then $\lim _{x \rightarrow \infty} \varphi(x)=0$ and $V_{\varphi}=\varphi$, whence (ii) follows.

Conversely, suppose that (ii) holds. By considering real and imaginary parts, we may assume that $\varphi$ is real. Then $\varphi=V_{\varphi}-\left(V_{\varphi}-\varphi\right)$, where $V_{\varphi}$ and $V_{\varphi}-\varphi$ are non-increasing functions in $X_{p}$; note that $0 \leq V_{\varphi}-\varphi \leq 2 V_{\varphi}$. Consequently (i) holds.

Since $V_{\varphi}$ is non-increasing, (ii) $\Leftrightarrow$ (iii) follows by Lemma 5.2.

Next, (iii) $\Leftrightarrow\left(\right.$ iv) follows easily because $V_{\varphi}(x)=\int_{x}^{\infty}|d \varphi(y)|$, and (iv) $\Leftrightarrow(\mathrm{v})$ is easily verified.

If (iv) holds, then $\|\varphi\|_{B V\left[2^{n}, 2^{n+1}\right]}=\int_{2^{n}}^{2^{n+1}}|d \varphi(x)|+\sup _{\left[2^{n}, 2^{n+1}\right]}|\varphi| \leq 2 \int_{2^{n}}^{\infty}|d \varphi(x)|$ and (vi) follows. Conversely, (vi) immediately implies (v).

Finally, for any functions $\varphi$ and $\psi$ on an interval $I,\|\psi \varphi\|_{B V(I)} \leq\|\psi\|_{B V(I)}\|\varphi\|_{B V(I)}$, and the equivalence (vi) $\Leftrightarrow$ (vii) follows by taking $\psi(x)=x$ and $\psi(x)=1 / x$.

We can define a norm in $Y_{p}$ (a quasi-norm for $p<1$ ) by

$$
\|\varphi\|_{Y_{p}} \stackrel{\text { def }}{=}\left(\sum_{n \in \mathbb{Z}} 2^{n p}\left(\int_{2^{n}}^{2^{n+1}}|d \varphi(x)|\right)^{p}\right)^{1 / p}
$$

an alternative is

$$
\left(\int_{0}^{\infty}\left|x V_{\varphi}(x)\right|^{p} \frac{d x}{x}\right)^{1 / p},
$$

which yields an equivalent (quasi-)norm.

We obtain as corollaries to Theorems 5.1 and 5.3 the following simple sufficient conditions for $Q_{\varphi} \in \boldsymbol{S}_{p}$.

Corollary 5.4. If $\varphi$ is absolutely continuous on $[0, \infty), \lim _{x \rightarrow \infty} \varphi(x)=0$ and $\sup _{x>0} x^{\gamma}\left|\varphi^{\prime}(x)\right|<\infty$ for some $\gamma>2$, then $\varphi \in Y_{p}$ for every $p>0$ and thus $Q_{\varphi} \in \boldsymbol{S}_{p}$ for every $p>1 / 2$.

Proof. $V_{\varphi}(x)$ is bounded and $\left|V_{\varphi}(x)\right| \leq$ const $\cdot x^{1-\gamma}$, and thus $x V_{\varphi}(x) \in L^{p}(d x / x)$ for every $p>0$. 
Corollary 5.5. If $\varphi$ has bounded variation and support in a finite interval, then $\varphi \in Y_{p}$ for every $p>0$ and thus $Q_{\varphi} \in \boldsymbol{S}_{p}$ for every $p>1 / 2$.

\section{6. $p=1$, first results}

Let us now consider the case $p=1$. We know already that $\varphi \in X_{1}$ is a necessary and $\varphi \in Y_{1}$ a sufficient condition for $Q_{\varphi} \in \boldsymbol{S}_{1}$. We will later see that neither condition is both necessary and sufficient (see the example following Theorem 6.5). We restate these results as follows.

Theorem 6.1. If $\varphi$ has locally bounded variation, $\int_{0}^{\infty} x|d \varphi(x)|<\infty$ and $\lim _{x \rightarrow \infty} \varphi(x)=0$, then $Q_{\varphi} \in \boldsymbol{S}_{1}$.

Proof. It follows from Theorem 5.3 and the calculation

$$
\int_{0}^{\infty} V_{\varphi}(x) d x=\int_{0}^{\infty} \int_{x}^{\infty}|d \varphi(y)| d x=\int_{0}^{\infty} y|d \varphi(y)|
$$

that the assumption is equivalent to $\varphi \in Y_{1}$, so the result follows from Theorem 5.1 .

Theorem 6.2. If $Q_{\varphi} \in \boldsymbol{S}_{1}$, then $\varphi \in X_{1}$. Furthermore, $\varphi \in L^{1}(0, \infty)$ and

$$
\operatorname{trace} Q_{\varphi}=\int_{0}^{\infty} \varphi(x) d x
$$

Proof. By the Remark at the end of $\S 3, Q_{\varphi} \in \boldsymbol{S}_{1} \Rightarrow \varphi \in X_{1}$. Next, $X_{1} \subset L^{1}(0, \infty)$, since by the Cauchy-Schwarz inequality and (1.3)

$$
\int_{0}^{\infty}|\varphi| \leq \sum_{n \in \mathbb{Z}} 2^{n / 2}\left(\int_{2^{n}}^{2^{n+1}}|\varphi(x)|^{2} d x\right)^{1 / 2}<\infty .
$$

Finally, the trace formula follows from Theorem 6.3 below, since with $k(x, y)=\varphi(\max \{x, y\})$ for $x, y>0$,

$$
\int_{-\infty}^{\infty} k(x, x+a) d x=\int_{|a|}^{\infty} \varphi(x) d x
$$

Remark. This theorem gives a formula for the trace of $Q_{\varphi}$ if that operator has a trace; i.e., if it is in the trace class, $\boldsymbol{S}_{1}$. The theorem also shows that if $Q_{\varphi}$ is in the trace class then we must have $\varphi \in X_{1}$. We will see later in this section that $\varphi \in X_{1}$ is not enough to insure that $Q_{\varphi}$ is in the trace class. However, we will see later, Corollary 10.2, that $\varphi \in X_{1}$ is sufficient to insure that $Q_{\varphi}$ and related operators do have a Dixmier trace. 
In the previous theorem we used following fact from $[\mathrm{A}]$, improving an earlier result in $[\mathrm{B}]$ :

Theorem 6.3. If $T$ is an integral operator on $L^{2}(\mathbb{R})$ of class $\boldsymbol{S}_{1}$ with kernel function $k$, then the function

$$
x \mapsto k(x, x+a), \quad x \in \mathbb{R},
$$

is in $L^{1}(\mathbb{R})$ for almost all $a \in \mathbb{R}$ and the function

$$
a \mapsto \int_{\mathbb{R}} k(x, x+a) d x, \quad a \in \mathbb{R},
$$

is almost everywhere equal to the Fourier transform $\mathcal{F} h$ of a function $h \in L^{1}(\mathbb{R})$, in particular, it coincides a.e. with a continuous function on $\mathbb{R}$. Moreover,

$$
\operatorname{trace} T=(\mathcal{F} h)(0) \text {. }
$$

Proof. It is sufficient to prove the result when $k(x, y)=f(x) g(y)$ with $f$ and $g$ in $L^{2}(\mathbb{R})$, in which case it can be verified straightforwardly, with $h(\xi)=(\mathcal{F} f)(\xi)(\mathcal{F} g)(-\xi)$.

We can reduce the estimation of $\left\|Q_{\varphi}\right\|_{S_{1}}$ to the estimation of $\left\|Q_{\varphi}^{I}\right\|_{S_{1}}$ for dyadic intervals $I$.

Theorem 6.4. Let $\varphi \in L_{\mathrm{loc}}^{1}\left(\mathbb{R}_{+}\right)$and let $I_{n}=\left[2^{n}, 2^{n+1}\right]$. Then $Q_{\varphi} \in \boldsymbol{S}_{1}$ if and only if $\varphi \in X_{1}$ and

$$
\sum_{n \in \mathbb{Z}}\left\|Q_{\varphi}^{I_{n}}\right\|_{S_{1}}<\infty
$$

Proof. Consider the sets $A^{(k)}$ defined by (3.4) and consider their symmetric images $A^{(-k)}$ about the line $\{(x, y): x=y\}$. As in (3.8) we have

$$
\sum_{k \geq 1}\left\|\mathcal{P}_{A^{(k)}} Q_{\varphi}\right\|_{S_{1}} \leq \mathrm{const} \sum_{n \in \mathbb{Z}}\left(2^{n} \int_{2^{n}}^{2^{n+1}}|\varphi(x)|^{2} d x\right)^{1 / 2}
$$

Similarly,

$$
\sum_{k \geq 1}\left\|\mathcal{P}_{A^{(-k)}} Q_{\varphi}\right\|_{S_{1}} \leq \mathrm{const} \sum_{n \in \mathbb{Z}}\left(2^{n} \int_{2^{n}}^{2^{n+1}}|\varphi(x)|^{2} d x\right)^{1 / 2} .
$$

It thus follows from $\varphi \in X_{1}$ that

$$
\mathcal{P}_{A} Q_{\varphi} \in \boldsymbol{S}_{1} \quad \text { and } \quad \mathcal{P}_{\breve{A}} Q_{\varphi} \in \boldsymbol{S}_{1} .
$$

where

$$
A=\bigcup_{k \geq 1} A^{(k)} \quad \text { and } \quad \breve{A}=\bigcup_{k \leq-1} A^{(k)}
$$


Consequently, using Theorem $6.2, Q_{\varphi} \in \boldsymbol{S}_{1}$ if and only if $\varphi \in X_{1}$ and $\mathcal{P}_{B} Q_{\varphi} \in \boldsymbol{S}_{1}$, where $B$ is defined by (3.1). Since the projections of the sets $B_{n}$ onto the coordinate axes are disjoint, and $\mathcal{P}_{B_{n}} Q_{\varphi}=Q_{\varphi}^{I_{n}}$, it follows that $\mathcal{P}_{B} Q_{\varphi} \in \boldsymbol{S}_{1}$ if and only if (6.1) holds.

Let $n \in \mathbb{Z}$. It is easy to see that $Q_{\varphi}^{I_{n}} \in \boldsymbol{S}_{1}$ if and only if $Q_{\varphi_{n}} \in \boldsymbol{S}_{1}$, where

$$
\varphi_{n}(x) \stackrel{\text { def }}{=} \begin{cases}\varphi\left(x+2^{n}\right), & x \in\left[0,2^{n}\right] \\ 0, & \text { otherwise. }\end{cases}
$$

Hence, the question of when $Q_{\varphi}$ belongs to $\boldsymbol{S}_{1}$ reduces to the question of estimating $\left\|Q_{\varphi}\right\|_{S_{1}}$ for functions $\varphi$ supported on finite intervals.

Remark. For $0<p<1$, it can similarly be shown that if $\varphi \in X_{p}$ and $\sum_{n \in \mathbb{Z}}\left\|Q_{\varphi}^{I_{n}}\right\|_{\boldsymbol{S}_{p}}^{p}<\infty$, then $Q_{\varphi} \in \boldsymbol{S}_{p}$. We do not know whether the converse holds.

We next show that $\varphi \in X_{1}$ is not sufficient for $Q_{\varphi} \in \boldsymbol{S}_{1}$.

Theorem 6.5. Let $\varphi_{N}(x)=e^{2 \pi \mathrm{i} N x} \chi_{[0,1]}(x)$ for $N=1,2, \ldots$ Then

$$
s_{n}\left(Q_{\varphi_{N}}\right) \asymp \min \left\{\frac{1}{n+1}, \frac{N}{(n+1)^{2}}\right\},
$$

and so

$$
\left\|Q_{\varphi_{N}}\right\|_{\boldsymbol{S}_{1}} \asymp \log (N+1) .
$$

Note that $\asymp$ means that the ratio of the two sides are bounded from above and below by positive constants (not depending on $n$ or $N$ ). Clearly,

$$
\left\|\varphi_{N}\right\|_{X_{1}}=\left\|\chi_{[0,1]}\right\|_{X_{1}}=C
$$

is independent of $N$. This shows, by the closed graph theorem, that

$$
\varphi \in X_{1} \nRightarrow Q_{\varphi} \in \boldsymbol{S}_{1}
$$

a concrete counterexample is given in the example following the proof of Theorem 6.5. Moreover, $\varphi_{N} \in X_{p}$ for any $p>0$, again with norm independent of $N$, so for every $p \leq 1$

$$
\varphi \in X_{p} \nRightarrow Q_{\varphi} \in \boldsymbol{S}_{1} \text { and } \varphi \in X_{p} \nRightarrow Q_{\varphi} \in \boldsymbol{S}_{p} .
$$

It also follows from Theorem 6.5 that

$$
\left\|Q_{\varphi_{N}}\right\|_{\boldsymbol{S}_{p}} \asymp N^{(1-p) / p}, \quad \frac{1}{2}<p<1 .
$$

Proof of Theorem 6.5. Since multiplication by a unimodular function is a unitary operator, the singular values of $Q_{\varphi_{N}}$ are the same as the singular values $s_{n}\left(T_{N}\right)$, where $T_{N}$ is the integral operator on $L^{2}[0,1]$ with kernel

$$
\begin{aligned}
e^{-\pi \mathrm{i} N x} \varphi_{N}(\max (x, y)) e^{-\pi \mathrm{i} N y} & =\exp (2 \pi \mathrm{i} N \max (x, y)-\pi \mathrm{i} N x-\pi \mathrm{i} N y) \\
& =\exp (\pi \mathrm{i} N|x-y|) .
\end{aligned}
$$


Let $g_{N}(x)=e^{\pi \mathrm{i} N|x|}$ for $|x| \leq 1$, and extend $g_{N}$ to a function on $\mathbb{R}$ with period 2 . Let $T_{N}^{\prime}$ be the integral operator on $L^{2}[-1,1]$ with kernel $g_{N}(x-y)$. If $I_{+}=[0,1]$, $I_{-}=[-1,0]$ and $A_{\alpha \beta}=I_{\alpha} \times I_{\beta}, \alpha, \beta \in\{+,-\}$, then $\mathcal{P}_{A_{++}} T_{N}^{\prime}=T_{N}$ and thus

$$
s_{n}\left(Q_{\varphi_{N}}\right)=s_{n}\left(T_{N}\right) \leq s_{n}\left(T_{N}^{\prime}\right) \text {. }
$$

Moreover, each $\mathcal{P}_{A_{\alpha \beta}} T_{N}^{\prime}$ is by a translation unitarily equivalent to either $T_{N}$ or the integral operator on $L^{2}[0,1]$ with kernel $g_{N}(x-y-1)=\exp (\pi \mathrm{i} N(1-|x-y|))=$ $(-1)^{N} \overline{g_{N}}(x-y)$, which has the same singular values. Hence, by $(2.1)$,

$$
s_{4 n}\left(T_{N}^{\prime}\right) \leq 4 s_{n}\left(T_{N}\right)=4 s_{n}\left(Q_{\varphi_{N}}\right) .
$$

$T_{N}^{\prime}$ is a convolution operator on the circle $\mathbb{R} / 2 \mathbb{Z}$, so the elements of the orthonormal basis $\left\{2^{-1 / 2} \exp (\pi \mathrm{i} k x)\right\}_{k=-\infty}^{\infty}$ in $L^{2}[-1,1]$ are eigenvectors with eigenvalues

$$
\begin{aligned}
\hat{g}_{N}(k) & \stackrel{\text { def }}{=} \int_{-1}^{1} g_{N}(x) e^{-\pi \mathrm{i} k x} d x=\int_{-1}^{1} e^{\pi \mathrm{i} N|x|-\pi \mathrm{i} k x} d x \\
& =\int_{-1}^{0} e^{-\pi \mathrm{i}(N+k) x} d x+\int_{0}^{1} e^{\pi \mathrm{i}(N-k) x} d x \\
& = \begin{cases}0, & k \equiv N \quad(\bmod 2), k \neq \pm N, \\
1, & k= \pm N, \\
\frac{-2}{\pi \mathrm{i}(N+k)}+\frac{-2}{\pi \mathrm{i}(N-k)}=\frac{4 \mathrm{i} N}{\pi\left(N^{2}-k^{2}\right)}, & k \equiv N+1 \quad(\bmod 2) .\end{cases}
\end{aligned}
$$

Consequently, the singular values $s_{n}\left(T_{N}\right)$ are the absolute values $\left|\hat{g}_{N}(k)\right|, k \in \mathbb{Z}$, arranged in decreasing order. This easily yields

$$
s_{n}\left(T_{N}^{\prime}\right) \asymp \min \left\{\frac{1}{n+1}, \frac{N}{(n+1)^{2}}\right\},
$$

and the result follows by (6.4) and (6.5).

Remark. A related method is used in a more general context in $\S 14$. Indeed, the estimates (6.2) and (6.3) follow easily from Theorem 14.10 (with the norm estimates implicit there).

Moreover, (6.2) and (6.3) also follow from the results in $\S 16$, obtained by a different method.

Example. For a concrete counterexample we let $N_{k} \geq 2$ be integers and define

$$
\varphi=\sum_{k=1}^{\infty} \exp \left(2 \pi \mathrm{i} 2^{k} N_{k} x\right) \chi_{\left(2^{-k}, 2^{1-k}\right)}(x) .
$$

Then $|\varphi|=\chi_{[0,1]}$; in particular, $\varphi \in X_{p}$ for every $p>0$.

On the other hand, for every $k$, by Lemma 2.1 and Theorem 6.5,

$$
\left\|Q_{\varphi}\right\|_{\boldsymbol{S}_{1}} \geq\left\|Q_{\varphi}^{\left[2^{-k}, 2^{1-k}\right]}\right\|_{\boldsymbol{S}_{1}}=2^{-k}\left\|Q_{\varphi_{N_{k}}}\right\|_{\boldsymbol{S}_{1}} \geq c 2^{-k} \log N_{k}
$$


Choosing $N_{k}=2^{3^{k}}$, we find that $Q_{\varphi} \notin \boldsymbol{S}_{1}$.

Other choices yield further interesting examples. Thus, $N_{k}=2^{k}$ yields a symbol $\varphi \in X_{1}$ but $\varphi \notin Y_{1}$ such that, by Theorem $6.4, Q_{\varphi} \in \boldsymbol{S}_{1}$. In fact, using the Remark followed by Theorem 6.5, we can conclude that $Q_{\varphi} \in \boldsymbol{S}_{p}$ for every $p>1 / 2$.

The choice $N_{k}=2^{k^{2}}$ yields a symbol $\varphi \in X_{1} \backslash Y_{1}$ such that $Q_{\varphi} \in \boldsymbol{S}_{1}$ but $Q_{\varphi} \notin \boldsymbol{S}_{p}$ for $p<1$.

Remark. Theorem 6.5 implies also that $\varphi \in X_{1}$ does not imply $Q_{\varphi} \in \boldsymbol{S}_{1, q}$ for any Schatten-Lorentz space $\boldsymbol{S}_{1, q}$ with $q<\infty$.

Let us prove now that the condition $Q_{\varphi} \in \boldsymbol{S}_{1}$ does not imply that $Q_{\varphi}^{+} \in \boldsymbol{S}_{1}$. Moreover, as previously shown by Nowak [No], we show that there are no non-zero operators $Q_{\varphi}^{+}$of class $\boldsymbol{S}_{1}$. (A more refined result will be given in Theorem 10.1.)

Theorem 6.6. Suppose that $\varphi \in L_{\mathrm{loc}}^{1}\left(\mathbb{R}_{+}\right)$. If $Q_{\varphi}^{+} \in \boldsymbol{S}_{1}$, then $\varphi$ is the zero function.

Proof. Suppose that $Q_{\varphi}^{+} \in \boldsymbol{S}_{1}$. Let $k$ be the kernel function of $Q_{\varphi}^{+}$, extended by 0 to $\mathbb{R}^{2}$, i.e.

$$
k(x, y)= \begin{cases}\varphi(x), & 0<y<x \\ 0, & \text { otherwise }\end{cases}
$$

Let $\Delta \subset \mathbb{R}_{+}$be a compact interval. Consider the operator $P_{\Delta} Q_{\varphi}^{+} P_{\Delta}$, where $P_{\Delta}$ is multiplication by $\chi_{\Delta}$. Clearly, $P_{\Delta} Q_{\varphi}^{+} P_{\Delta}$ is an integral operator with kernel function $k_{\Delta} \stackrel{\text { def }}{=} \chi_{\Delta \times \Delta} k$, (recall that $\chi_{A}$ is the characteristic function of a set $A$ ) and $P_{\Delta} Q_{\varphi}^{+} P_{\Delta} \in \boldsymbol{S}_{1}$. It follows from Theorem 6.3 that the function $u$

$$
u(a) \stackrel{\text { def }}{=} \int_{\mathbb{R}} k_{\Delta}(x, x+a) d x
$$

is a.e. equal to a continuous function $\mathbb{R}$. Clearly, $u(a)=0$ if $a>0$. And

$$
u(a) \rightarrow \int_{\Delta} \varphi(x) d x
$$

when $a \uparrow 0$. Hence,

$$
\int_{\Delta} \varphi(x) d x=0, \quad \text { for any interval } \Delta \subset \mathbb{R}_{+} .
$$

Consequently, $\varphi=0$. 


\section{Schur multipliers of the form $\psi(\max \{x, y\})$, $\boldsymbol{x}, \boldsymbol{y} \in \mathbb{R}_{+}$}

Let $0<p \leq 2$. Recall that a function $\omega$ on $\mathbb{R}^{2}$ is called a Schur multiplier of $\boldsymbol{S}_{p}$ if the integral operator on $L^{2}(\mathbb{R})$ with kernel function $\omega k$ belongs to $\boldsymbol{S}_{p}$ whenever the integral operator with kernel function $k$ does. If $2<p<\infty$, the class of Schur multipliers of $\boldsymbol{S}_{p}$ can be defined by duality: $\omega$ is a Schur multiplier of $\boldsymbol{S}_{p}$ if $\omega$ is a Schur multiplier of $\boldsymbol{S}_{p^{\prime}}$, where $p^{\prime} \stackrel{\text { def }}{=} p /(p-1)$. We say that $\omega$ is a Schur multiplier of weak type $(p, p), 0<p \leq 2$, if the integral operator with kernel function $\omega k$ belongs to $\boldsymbol{S}_{p, \infty}$ whenever the integral operator with kernel function $k$ belongs to $\boldsymbol{S}_{p}$. Note that in a similar way one can define Schur multipliers for an arbitrary measure space $(\mathcal{X}, \mu)$.

In this section for a function $\psi \in L^{\infty}(\mathbb{R})$ we find a sufficient condition for the function $(x, y) \mapsto \psi(\max \{x, y\}),(x, y) \in \mathbb{R}^{2}$, to be a Schur multiplier of $\boldsymbol{S}_{p}$. We also obtain a sufficient condition for this function to be a Schur multiplier of weak type $(1 / 2,1 / 2)$.

Theorem 7.1. Let $1<p<\infty$ and let $\psi \in L^{\infty}(\mathbb{R})$. Then the function

$$
(x, y) \mapsto \psi(\max \{x, y\}), \quad(x, y) \in \mathbb{R}^{2},
$$

is a Schur multiplier of $\boldsymbol{S}_{p}$.

Proof. Since the triangular projection $\mathcal{P}$ is bounded on $\boldsymbol{S}_{p}$, the characteristic function of the set $\{(x, y): x>y\}$ is a Schur multiplier of $\boldsymbol{S}_{p}$. It remains to observe that

$$
\psi(\max \{x, y\})=\psi(x) \chi_{\{(x, y): x>y\}}+\psi(y) \chi_{\{(x, y): x<y\}} .
$$

Theorem 7.2. Let $\psi \in L^{\infty}(\mathbb{R})$. Then the function

$$
(x, y) \mapsto \psi(\max \{x, y\}), \quad(x, y) \in \mathbb{R}^{2},
$$

is a Schur multiplier of weak type $(1,1)$.

Proof. The result follows from (7.1) and the fact that the triangular projection $\mathcal{P}$ has weak type $(1,1)$ (see $(2.9))$.

Theorem 7.3. Let $1 / 2<p<\infty$ and let $\psi$ be a function of bounded variation. Then the function $(x, y) \mapsto \psi(\max \{x, y\})$ on $\mathbb{R}^{2}$ is a Schur multiplier of $\boldsymbol{S}_{p}$.

Proof. By Theorem 7.1 we may assume that $p \leq 1$.

We consider first the case when $\psi$ is absolutely continuous, i.e.,

$$
\psi(x)=\int_{x}^{\infty} h(t) d t+C, \quad h \in L^{1}(\mathbb{R}) .
$$


We may assume that $C=0$.

Let $\xi$ and $\eta$ be functions in $L^{2}$ and let $T$ be the integral operator defined by

$$
(T f)(x)=\int_{\mathbb{R}} \xi(x) \eta(y) \psi(\max \{x, y\}) f(y) d y, \quad f \in L^{2}(\mathbb{R}) .
$$

We have to prove that

$$
\|T\|_{\boldsymbol{S}_{p}} \leq C(p, h)\|\xi\|_{L^{2}(\mathbb{R})}\|\eta\|_{L^{2}(\mathbb{R})},
$$

where $C(p, h)$ may depend only on $p$ and $h$.

We can factorize the function $h$ in the form $h=u v$, where $u, v \in L^{2}(\mathbb{R})$. Put

$$
k_{1}(x, y) \stackrel{\text { def }}{=} \begin{cases}0, & y<x \\ \xi(x) u(y), & y>x\end{cases}
$$

and

$$
k_{2}(x, y) \stackrel{\text { def }}{=} \begin{cases}\eta(y) v(x), & y<x \\ 0, & y>x\end{cases}
$$

Let $T_{1}$ and $T_{2}$ be the integral operators on $L^{2}(\mathbb{R})$ with kernel functions $k_{1}$ and $k_{2}$. It follow from the boundedness of the triangular projection that if $1<q<\infty$, then $\left\|T_{1}\right\|_{S_{q}} \leq C(q)\|\xi\|_{L^{2}}\|u\|_{L^{2}}$ and $\left\|T_{2}\right\|_{S_{q}} \leq C(q)\|\eta\|_{L^{2}}\|v\|_{L^{2}}$, where $C(q)$ may depend only on $q$. It is also easy to verify that $T=T_{1} T_{2}$. It follows that

$$
\|T\|_{\boldsymbol{S}_{p}} \leq\left\|T_{1}\right\|_{\boldsymbol{S}_{2 p}}\left\|T_{2}\right\|_{\boldsymbol{S}_{2 p}} \leq(C(2 p))^{2}\|\xi\|_{L^{2}}\|\eta\|_{L^{2}}\|u\|_{L^{2}}\|v\|_{L^{2}} .
$$

To reduce the general case to the case of an absolutely continuous function $\psi$, we can consider a standard regularization process.

We complete this section with the following result.

Theorem 7.4. Suppose that $\psi$ is a function of bounded variation. Then the function $(x, y) \mapsto \psi(\max \{x, y\})$ is a Schur multiplier of weak type $(1 / 2,1 / 2)$.

We need two lemmata.

Lemma 7.5. Let $0<p<1$ and let $A \in \boldsymbol{S}_{p, \infty}$. Set

$$
\|A\|_{\boldsymbol{S}_{p, \infty}}^{*} \stackrel{\text { def }}{=} \sup _{t>0}\left(t^{p-1} \sum_{n \geq 0} \min \left\{t, s_{n}(A)\right\}\right)^{1 / p} .
$$

Then

$$
\|A\|_{\boldsymbol{S}_{p, \infty}} \leq\|A\|_{\boldsymbol{S}_{p, \infty}}^{*} \leq(1-p)^{-1 / p}\|A\|_{\boldsymbol{S}_{p, \infty}} .
$$

Proof. Taking $t=s_{n}(A)$ in the definition of $\|\cdot\|_{S_{p, \infty}}^{*}$, we obtain

$$
(n+1)^{\frac{1}{p}} s_{n}(A) \leq\|A\|_{S_{p, \infty}}^{*} .
$$


Consequently, $\|A\|_{\boldsymbol{S}_{p, \infty}} \leq\|A\|_{\boldsymbol{S}_{p, \infty}}^{*}$. Next, we have

$$
\begin{aligned}
t^{p-1} \sum_{n \geq 0} \min \left\{t, s_{n}(A)\right\} & \leq t^{p-1} \sum_{n \geq 0} \min \left\{t,\|A\|_{\boldsymbol{S}_{p, \infty}}(n+1)^{-\frac{1}{p}}\right\} \\
& \leq t^{p-1} \int_{0}^{\infty} \min \left\{t,\|A\|_{\boldsymbol{S}_{p, \infty}} x^{-1 / p}\right\} d x=(1-p)^{-1}\|A\|_{\boldsymbol{S}_{p, \infty}}^{p} .
\end{aligned}
$$

Hence, $\|A\|_{\boldsymbol{S}_{p, \infty}}^{*} \leq(1-p)^{-1 / p}\|A\|_{\boldsymbol{S}_{p, \infty}}$.

Lemma 7.6. If $0<p<1$, then $\|\cdot\|_{\boldsymbol{S}_{p, \infty}}^{*}$ is a p-norm, i.e.,

$$
\left\|A_{1}+A_{2}\right\|_{\boldsymbol{S}_{p, \infty}}^{* p} \leq\left\|A_{1}\right\|_{\boldsymbol{S}_{p, \infty}}^{* p}+\left\|A_{2}\right\|_{\boldsymbol{S}_{p, \infty}}^{* p}, \quad A_{1}, A_{2} \in \boldsymbol{S}_{p, \infty} .
$$

Proof. By Rotfeld's theorem [R], if $\Phi$ is a concave nondecreasing function on $\mathbb{R}_{+}$such that $\Phi\left(0^{+}\right)=0$, then

$$
\sum_{j=0}^{m} \Phi\left(s_{j}\left(A_{1}+A_{2}\right)\right) \leq \sum_{j=0}^{m} \Phi\left(s_{j}\left(A_{1}\right)\right)+\sum_{j=0}^{m} \Phi\left(s_{j}\left(A_{2}\right)\right), \quad m \in \mathbb{Z}_{+} .
$$

For $t>0$ we define the function $\Phi_{t}$ on $\mathbb{R}_{+}$by

$$
\Phi_{t}(x)=t^{p-1} \min \{t, x\} .
$$

Clearly,

$$
\|A\|_{\boldsymbol{S}_{p, \infty}}^{* p}=\sup _{t>0} \sum_{j \geq 0} \Phi_{t}\left(s_{j}(A)\right) .
$$

It remains to apply 7.4 for $\Phi_{t}$ and take the supremum over $t>0$.

Note that the fact that for $p<1$ the space $L^{p, \infty}$ has a $p$-norm that is equivalent to the initial quasi-norm is well known (see $[\mathrm{K}]$ ).

Proof of Theorem 7.4. The proof is similar to the proof of Theorem 7.3. Again, it is sufficient to assume that $\psi$ has the form (7.2) with $C=0$.

By Lemma 7.5 and Lemma 7.6, to prove that our function is a Schur multiplier of weak type $(1 / 2,1 / 2)$, it is sufficient to prove that if $T$ is defined by (7.3), then $T \in \boldsymbol{S}_{1 / 2, \infty}$. Let $u, v, k_{1}, k_{2}, T_{1}$, and $T_{2}$ be as in the proof of Theorem 7.3. Let $n \geq 2$ and $n=m_{1}+m_{2}$, where $\left|m_{1}-n / 2\right| \leq 1 / 2$ and $\left|m_{2}-n / 2\right| \leq 1 / 2$. Since the triangular projection $\mathcal{P}$ has weak type $(1,1)$ (see $(2.9)$ ), we have

$$
\left\|T_{1}\right\|_{\boldsymbol{S}_{1, \infty}} \leq \mathrm{const}\|\xi\|_{L^{2}}\|u\|_{L^{2}} \quad \text { and } \quad\left\|T_{2}\right\|_{\boldsymbol{S}_{1, \infty}} \leq \mathrm{const}\|\eta\|_{L^{2}}\|v\|_{L^{2}} .
$$

Hence, by (2.2),

$$
\begin{aligned}
s_{n}(T) & \leq s_{m_{1}}\left(T_{1}\right) s_{m_{2}}\left(T_{2}\right) \leq \mathrm{const} \frac{1}{m_{1} m_{2}}\|\xi\|_{L^{2}}\|u\|_{L^{2}}\|\eta\|_{L^{2}}\|v\|_{L^{2}} \\
& \leq \mathrm{const} \frac{1}{n^{2}}\|\xi\|_{L^{2}}\|u\|_{L^{2}}\|\eta\|_{L^{2}}\|v\|_{L^{2}}
\end{aligned}
$$


which completes the proof.

We let $\mathfrak{M}_{p}$ denote the space of Schur multipliers of $\boldsymbol{S}_{p}$, and put

$$
\|\omega\|_{\mathfrak{M}_{p}} \stackrel{\text { def }}{=} \sup \|\omega k\|_{\boldsymbol{S}_{p}}
$$

where the supremum is taken over all integral operators with kernel $k \in L^{2}$ such that $\|k\|_{\boldsymbol{S}_{p}}=1$. Here by $\|k\|_{\boldsymbol{S}_{p}}$ we mean the $S_{p}$ norm (quasi-norm if $p<1$ ) of the integral operator with kernel function $k$. If $\omega$ is a Schur multiplier of weak type $(p, p)$, we put

$$
\|\omega\|_{\mathfrak{M}_{p, \mathrm{w}}} \stackrel{\text { def }}{=} \sup \|\omega k\|_{\boldsymbol{S}_{p, \infty}}
$$

where the supremum is taken over all integral operators with kernel $k \in L^{2}$ such that $\|k\|_{S_{p}}=1$.

Remark. It is clear from the proofs of Theorems 7.1, 7.2, 7.3, and 7.4 that under the hypotheses of Theorem 7.1 we have

$$
\|\psi(\max \{x, y\})\|_{\mathfrak{M}_{p}} \leq C(p)\|\psi\|_{L^{\infty}}
$$

and

$$
\|\psi(\max \{x, y\})\|_{\mathfrak{M}_{1, \mathrm{w}}} \leq C\|\psi\|_{L^{\infty}}
$$

while under the hypotheses of Theorem 7.3 we have

$$
\|\psi(\max \{x, y\})\|_{\mathfrak{M}_{p}} \leq C(p)\|\psi\|_{B V},
$$

and

$$
\|\psi(\max \{x, y\})\|_{\mathfrak{M}_{1 / 2, \mathrm{w}}} \leq C\|\psi\|_{B V} .
$$

Here we use the notation

$$
\|\varphi\|_{B V}^{\prime}=\int_{\mathbb{R}}|d \varphi| \text { and }\|\varphi\|_{B V}=\|\varphi\|_{B V}^{\prime}+\|\varphi\|_{L^{\infty}} .
$$

The following result gives us a more accurate estimate for $\|\psi(\max \{x, y\})\|_{\mathfrak{M}_{p}}$ in the case $1 / 2<p \leq 1$.

Theorem 7.7. Let $\psi$ be a function of bounded variation on $\mathbb{R}$. Then

$$
\|\psi(\max \{x, y\})\|_{\mathfrak{M}_{1}} \leq \mathrm{const}\|\psi\|_{L^{\infty}} \log \left(2+\frac{\|\psi\|_{B V}^{\prime}}{\|\psi\|_{L^{\infty}}}\right)
$$

and

$$
\|\psi(\max \{x, y\})\|_{\mathfrak{M}_{p}} \leq \mathrm{const}\|\psi\|_{L^{\infty}}^{2-1 / p}\|\psi\|_{B V}^{1 / p-1}, \quad \frac{1}{2}<p<1 .
$$

Proof. Let $\xi$ and $\eta$ be function in $L^{2}$ such that $\|\xi\|_{L^{2}}=\|\eta\|_{L^{2}}=1$. We have to estimate the $\boldsymbol{S}_{p}$-norm of the integral operator with kernel $\psi(\max \{x, y\}) \xi(x) \eta(y)$. Let $\left\{s_{n}\right\}_{n \geq 0}$ be the sequence of $s$-numbers of this integral operator. Theorem 7.2 implies that

$$
s_{n} \leq \mathrm{const} \frac{\|\psi\|_{L^{\infty}}}{n+1}
$$


Theorem 7.4 implies that

$$
s_{n} \leq \mathrm{const} \frac{\|\psi\|_{L^{\infty}}+\|\psi\|_{B V}^{\prime}}{(n+1)^{2}} .
$$

Consequently,

$$
s_{n} \leq \text { const } \min \left\{\frac{\|\psi\|_{L^{\infty}}}{n+1}, \frac{\|\psi\|_{L^{\infty}}+\|\psi\|_{B V}^{\prime}}{(n+1)^{2}}\right\} .
$$

The rest of the proof is an easy exercise.

\section{The case $p=1 / 2$}

Theorem 8.1. Let $\varphi$ be a function of bounded variation on $[0,1]$. Then $Q_{\varphi}^{[0,1]} \in \boldsymbol{S}_{1 / 2, \infty}$.

Proof. We may extend the function $\varphi$ by putting $\varphi(t)=0$ for $t \in \mathbb{R}_{+} \backslash[0,1]$. Clearly, the integral operator with kernel function $\chi_{[0,1]^{2}}$ has rank one, and so it belongs to $\boldsymbol{S}_{1 / 2}$. Consequently, by Theorem 7.4, the integral operator with kernel function $\chi_{[0,1]^{2}} \varphi(\max \{x, y\})$ belongs to $\boldsymbol{S}_{1 / 2, \infty}$.

To see that this result cannot be improved to $Q_{\varphi}^{[0,1]} \in \boldsymbol{S}_{1 / 2}$, we begin with two extensions of Theorem 6.6.

Lemma 8.2. Let $\varphi, \psi \in L_{\text {loc }}^{1}(\mathbb{R})$. Suppose that the integral operator with kernel function $k$,

$$
k(x, y)= \begin{cases}\varphi(x) \psi(y), & y \leq x, \\ 0, & \text { otherwise, }\end{cases}
$$

belongs to $\boldsymbol{S}_{1}$. Then $\varphi \psi=0$ almost everywhere.

Proof. First we assume that $\varphi, \psi \in L^{2}(\mathbb{R})$. By Theorem 6.3 we have

$$
\lim _{a \rightarrow 0-} \int_{\mathbb{R}} k(x, x+a) d x=\lim _{a \rightarrow 0+} \int_{\mathbb{R}} k(x, x+a) d x=0,
$$

whence $\int_{\mathbb{R}} \varphi(x) \psi(x) d x=0$. Now let $\varphi$ and $\psi$ be arbitrary functions in $L_{\text {loc }}^{1}(\mathbb{R})$. Suppose that $f$ and $g$ are functions in $L^{\infty}(\mathbb{R})$ such that $f \varphi \in L^{2}(\mathbb{R})$ and $g \psi \in L^{2}(\mathbb{R})$. Consider the integral operator with kernel function

$$
(x, y) \mapsto f(x) k(x, y) g(y) .
$$

Clearly, it belongs to $\boldsymbol{S}_{1}$. It follows from what we have just proved that $\int_{\mathbb{R}} \varphi(x) f(x) \psi(x) g(x) d x=0$. Since $f$ and $g$ are arbitrary, this implies the result. 
Lemma 8.3. Let $\varphi \in L_{\text {loc }}^{1}\left(\mathbb{R}_{+}\right)$and let $A=(0, \infty) \times \Delta$, where $\Delta$ is a measurable subset of $(0, \infty)$. Suppose that $\mathcal{P}_{A} Q_{\varphi}^{+} \in \boldsymbol{S}_{1}$. Then $\varphi=0$ almost everywhere on $\Delta$.

Proof. The result follows easily from Lemma 8.2 with $\psi=\chi_{\Delta}$.

Lemma 8.4. Let $\varphi$ be a nonincreasing locally absolutely continuous function on $\mathbb{R}_{+}$and let $\Delta$ be a measurable subset of $\mathbb{R}_{+}$. Suppose that the integral operator with kernel function

$$
(x, y) \mapsto \varphi(\max \{x, y\}) \chi_{\Delta}(x) \chi_{\Delta}(y)
$$

belongs to $\boldsymbol{S}_{1 / 2}$. Then $\varphi^{\prime}=0$ almost everywhere on $\Delta$.

Proof. By replacing $\Delta$ with $\Delta \cap(a, b)$, we may assume that $\Delta \subset[a, b]$ where $0<a<b<\infty$. We may then subtract $\varphi(b)$ and modify $\varphi$ outside $[a, b]$ so that $\varphi$ becomes constant on $(0, a]$ and zero on $[b, \infty)$. Let $\psi=\left(-\varphi^{\prime}\right)^{1 / 2}$. Since $x \varphi(x)$ is bounded, we have $\psi \in X_{\infty}$. Thus $Q_{\psi}^{+}$is bounded and by Theorem $4.2, Q_{\varphi}$ admits a factorization $Q_{\varphi}=\left(Q_{\psi}^{+}\right)^{*} Q_{\psi}^{+}$. Let $M$ be multiplication by $\chi_{\Delta}$. It follows that $M Q_{\varphi} M=\left(Q_{\psi}^{+} M\right)^{*}\left(Q_{\psi}^{+} M\right)$, and so $Q_{\psi}^{+} M \in \boldsymbol{S}_{1}$. The result follows now from Lemma 8.3.

Corollary 8.5. Let $\varphi$ be as in Lemma 8.4 and let $\psi$ be a function on $\mathbb{R}_{+}$such that $Q_{\psi} \in \boldsymbol{S}_{1 / 2}$. Set $\Delta \stackrel{\text { def }}{=}\left\{x \in \mathbb{R}_{+}: \psi(x)=\varphi(x)\right\}$. Suppose that $\psi$ is differentiable almost everywhere on $\Delta$. Then $\varphi^{\prime}=\psi^{\prime}=0$ almost everywhere on $\Delta$.

Proof. It suffices to apply Lemma 8.4.

Theorem 8.6. Let $\psi$ be an absolutely continuous function on $[0,1]$. Suppose $Q_{\psi}^{[0,1]} \in \boldsymbol{S}_{1 / 2}$. Then $\psi$ is constant.

Proof. Clearly, we may assume that $\psi$ is a real function. Suppose that $\psi$ is not constant. Then $\max \psi>\psi(1)$ or $\min \psi<\psi(1)$. To be definite, suppose that $\max \psi>\psi(1)$. We use the "sun rising method". Let

$$
\Delta \stackrel{\text { def }}{=}\{x \in[0,1]: \psi(x) \geq \psi(t) \text { for all } t \geq x\} .
$$

Clearly, $\Delta$ is closed and $1 \in \Delta$. Moreover, the restriction $\psi \mid[\alpha, \beta]$ is constant for any interval $(\alpha, \beta)$ such that $\alpha, \beta \in \Delta$ and $\Delta \cap(\alpha, \beta)=\varnothing$. Set

$$
\varphi(x)=\max _{[x, 1] \cap \Delta} \psi .
$$

Clearly, $\varphi$ is non-increasing, $\left.\varphi\right|_{\Delta}=\left.\psi\right|_{\Delta}$ and $\varphi \mid[\alpha, \beta]$ is constant for any interval $(\alpha, \beta)$ such that $\alpha, \beta \in \Delta$ and $\Delta \cap(\alpha, \beta)=\varnothing$. Consequently, $\varphi$ is absolutely continuous. Thus, $\varphi^{\prime}=0$ almost everywhere on $\Delta$ by Corollary 8.5. Moreover, $\varphi^{\prime}=0$ outside $\Delta$ because $\varphi$ is locally constant outside $\Delta$. Consequently, $\varphi^{\prime}=0$ almost everywhere on $[0,1]$, and so $\varphi(t)=\varphi(1)=\psi(1)$ for any $t \in[0,1]$ which contradicts the condition $\max \psi>\psi(1)$. 
Corollary 8.7. Suppose that $Q_{\psi} \in \boldsymbol{S}_{1 / 2}$. Then $\psi$ is constant on any interval $I \subset \mathbb{R}_{+}$on which $\psi$ is absolutely continuous.

Corollary 8.8. Suppose that $\psi$ is locally absolutely continuous and $Q_{\psi} \in \boldsymbol{S}_{1 / 2}$. Then $\psi=0$ everywhere on $\mathbb{R}_{+}$.

Lemma 8.9. Let $\varphi$ be a nonincreasing function on $\mathbb{R}_{+}$with $\lim _{t \rightarrow+\infty} \varphi(t)=0$ and let $\varepsilon>0$. Then there exists a nonincreasing absolutely continuous function $\psi$ on $\mathbb{R}_{+}$such that $\lim _{t \rightarrow+\infty} \psi(t)=0$ and $\boldsymbol{m}\{\varphi \neq \psi\}<\varepsilon$.

Note that here and in what follows $\boldsymbol{m}$ denotes Lebesgue measure on $\mathbb{R}$ or normalized Lebesgue measure on the unit circle $\mathbb{T}$.

Proof. We may assume that $\varphi$ is right-continuous on $(0, \infty)$ and that $\varphi$ is bounded. Consider the positive measure $\mu$ on $\mathbb{R}_{+}$such that $\varphi(t)=\mu(t, \infty)$ for any $t>0$. Denote by $\mu_{s}$ the singular part of $\mu$. There exists a Borel set $E \subset \mathbb{R}_{+}$ such that $\boldsymbol{m}(E)=0$ and $\mu_{s}((0,+\infty) \backslash E)=0$. We may find an open set $U$ such that $E \subset U \subset \mathbb{R}_{+}$and $\mu(U)<\varepsilon$. Let $U=\bigcup_{n \geq 1}\left(a_{n}, b_{n}\right)$, where $\left(a_{n}, b_{n}\right)$ are mutually disjoint. Set

$$
f(t)= \begin{cases}-\varphi^{\prime}(t), & t \in \mathbb{R}_{+} \backslash E, \\ \frac{\mu\left[a_{n}, b_{n}\right)}{b_{n}-a_{n}}, & t \in\left(a_{n}, b_{n}\right) .\end{cases}
$$

Set $\psi(t) \stackrel{\text { def }}{=} \int_{t}^{\infty} f(s) d s$. Clearly, $\varphi=\psi$ outside $U$.

Lemma 8.10. Let $\varphi$ a nonincreasing function on $\mathbb{R}_{+}$with $\lim _{t \rightarrow+\infty} \varphi(t)=0$. Suppose that the integral operator with kernel function

$$
(x, y) \mapsto \varphi(\max \{x, y\}) \chi_{\Delta}(x) \chi_{\Delta}(y)
$$

belongs to $\boldsymbol{S}_{1 / 2}$ for a measurable subset $\Delta$ of $\mathbb{R}_{+}$. Then $\varphi^{\prime}=0$ almost everywhere on $\Delta$.

Proof. The result follows from Lemmas 8.4 and 8.9.

Theorem 8.11. Let $\psi$ be a function with bounded variation on $[0,1]$. Suppose that $Q_{\psi}^{[0,1]} \in \boldsymbol{S}_{1 / 2}$. Then $\psi^{\prime}=0$ almost everywhere on $[0,1]$.

Proof. Again, we may assume that $\psi$ is real. We may also make the assumption that that $\psi$ is continuous at 0 and at 1 , and $\psi(t)=\max \left\{\psi\left(t^{-}\right), \psi\left(t^{+}\right)\right\}$for any $t \in(0,1)$. With any nondegenerate closed interval $I \subset[0,1]$ we associate the function $\varphi_{I}: I \rightarrow \mathbb{R}$ defined by $\varphi_{I}(x)=\sup \{\psi(t): t \in I$ and $t \geq x\}$. Set

$$
\Delta(I) \stackrel{\text { def }}{=}\left\{x \in I: \varphi_{I}(x)=\psi(x)\right\} .
$$


Clearly, $\Delta(I)$ is closed. By Lemma 8.10, $\psi^{\prime}=\varphi_{I}^{\prime}=0$ almost everywhere on $\Delta(I)$.

Set $E_{-}=\left\{x \in(0,1): \psi^{\prime}(x)<0\right\}$. Let $a \in E_{-}$. Clearly, $a \in \Delta(I)$ if $I$ is small enough and $a \in I$. Consequently,

$$
E_{-} \subset \bigcup_{n=2}^{\infty}\left(\bigcup_{k=1}^{n-1} \Delta\left(\left[\frac{k-1}{n}, \frac{k+1}{n}\right]\right)\right) \text {. }
$$

We have shown that Lemma 8.10 implies $\boldsymbol{m}\left(\Delta(I) \cap E_{-}\right)=0$ for every $I$. Consequently, $\boldsymbol{m}\left(E_{-}\right)=0$. Thus, we have proved that $\psi^{\prime} \geq 0$ almost everywhere. It remains to apply this result to $-\psi$.

The following fact is an immediate consequence of Theorem 8.11.

Corollary 8.12. Suppose that $Q_{\psi} \in \boldsymbol{S}_{1 / 2}$. Then $\psi^{\prime}=0$ almost everywhere on any interval $I \subset \mathbb{R}_{+}$on which $\psi$ is of bounded variation.

\section{Sturm-Liouville theory and $p=1 / 2$}

If $\varphi$ is real, then $Q_{\varphi}$ is self-adjoint, so its singular values are the absolute values of its eigenvalues. Hence, we next study the eigenvalues and eigenfunctions. For simplicity we consider only the case of symbols $\varphi$ which vanish on $(1, \infty)$; thus it does not matter whether we consider $Q_{\varphi}$ on $L^{2}\left(\mathbb{R}_{+}\right)$or $Q_{\varphi}^{[0,1]}$ on $L^{2}[0,1]$.

Suppose that $\varphi \in C^{1}[0,1]$ and that $\varphi$ is real. Let $\lambda$ be a non-zero eigenvalue of $Q_{\varphi}^{[0,1]}$ and $g \in L^{2}[0,1]$ a corresponding eigenfunction,

$$
\lambda g(x)=\varphi(x) \int_{0}^{x} g(y) d y+\int_{x}^{1} \varphi(y) g(y) d y, \quad 0 \leq x \leq 1 .
$$

The right hand side is a continuous function of $x$; hence, $g \in C[0,1]$ and (9.1) holds for every $x$ (and not just a.e.). By (9.1) again, $g \in C^{1}[0,1]$ with

$$
\lambda g^{\prime}(x)=\varphi^{\prime}(x) \int_{0}^{x} g(y) d y .
$$

Define $G(x)=\int_{0}^{x} g(y) d y$. Then (9.2) can be written as the system

$$
\begin{aligned}
G^{\prime}(x) & =g(x) \\
g^{\prime}(x) & =\lambda^{-1} \varphi^{\prime}(x) G(x)
\end{aligned}
$$

and we have, using (9.1) with $x=1$, the boundary conditions

$$
\begin{aligned}
G(0) & =0, \\
g(1) & =\lambda^{-1} \varphi(1) G(1) .
\end{aligned}
$$


Conversely, any solution of (9.3) with the boundary conditions (9.4) satisfies (9.2) and (9.1), so the problem of finding the singular values of $Q_{\varphi}$ reduces to finding the $\lambda \neq 0$ for which (9.3) and (9.4) have a solution. Note that (9.3) can be written as a Sturm-Liouville problem

$$
\lambda G^{\prime \prime}(x)=\varphi^{\prime}(x) G(x) .
$$

If $g\left(x_{0}\right)=G\left(x_{0}\right)=0$ for some $x_{0} \in[0,1]$, then (9.3) shows, by the standard uniqueness theorem, that $g$ vanishes identically, a contradiction. In particular, since $G(0)=0$, we have $g(0) \neq 0$, and we may normalize the eigenfunction $g$ by $g(0)=1$.

For every $\lambda \neq 0,(9.3)$ has a unique solution $\left(G_{\lambda}, g_{\lambda}\right)$ with $G_{\lambda}(0)=0, g_{\lambda}(0)=1$. It thus follows that all non-zero eigenvalues of $Q_{\varphi}$ are simple, and that $\lambda \neq 0$ is an eigenvalue if and only if

$$
g_{\lambda}(1)=\lambda^{-1} \varphi(1) G_{\lambda}(1) .
$$

Example. Let $\varphi(x)=1-x, x \in[0,1]$, and $\varphi(x)=0, x>1$. Then (9.3) gives $G^{\prime \prime}(x)=-\lambda^{-1} G(x)$, and we find the solutions $g_{\lambda}(x)=\cos \lambda^{-1 / 2} x, \lambda>0$, and $g_{\lambda}(x)=\cosh |\lambda|^{-1 / 2} x, \lambda<0$.

Since $\varphi(1)=0$, condition (9.6) is simply $g_{\lambda}(1)=0$, and the non-zero eigenvalues are given by $\cos \lambda^{-1 / 2}=0$ or $\lambda^{-1 / 2}=\left(n+\frac{1}{2}\right) \pi, n=0,1, \ldots(\lambda<0$ is impossible in this case; in other words, $Q_{\varphi}$ is a positive operator, as is also seen by Theorem 4.1.) Hence, the non-zero eigenvalues are $\left\{\left(n+\frac{1}{2}\right)^{-2} \pi^{-2}\right\}_{n=0}^{\infty}$ and the singular values are $s_{n}=\pi^{-2}\left(n+\frac{1}{2}\right)^{-2}, n \geq 0$.

The behaviour $s_{n}\left(Q_{\varphi}\right) \asymp(n+1)^{-2}$ found in the above example holds for all smooth $\varphi$ on $[0,1]$ by Sturm-Liouville theory, as will be seen in Theorem 9.3. Hence, for smooth $\varphi$ with compact support, we have $Q_{\varphi} \in \boldsymbol{S}_{1 / 2, \infty}$ but nothing better.

Let $B V[0,1]$ denote the Banach space of functions on $[0,1]$ with bounded variation, with the seminorm $\|\varphi\|_{B V}^{\prime}=\int_{0}^{1}|d \varphi|$ and the norm $\|\varphi\|_{B V}=\|\varphi\|_{B V}^{\prime}+\sup |\varphi|$.

The following result is essentially the same as Theorem 8.1. However, we use in this section a different approach based on the study of eigenvalues of SturmLiouville operators.

Theorem 9.1. If $\varphi \in B V[0,1]$, and $\varphi=0$ on $(1, \infty)$, then $Q_{\varphi} \in \boldsymbol{S}_{1 / 2, \infty}$ and $\left\|Q_{\varphi}\right\|_{S_{1 / 2, \infty}} \leq C\|\varphi\|_{B V}$. More precisely,

$$
s_{n}\left(Q_{\varphi}\right) \leq C_{1}\|\varphi\|_{B V}(n+1)^{-2}, \quad n \geq 0,
$$

and

$$
s_{n}\left(Q_{\varphi}\right) \leq C_{2}\|\varphi\|_{B V}^{\prime} n^{-2}, \quad n \geq 1
$$


Proof. Note that (9.8) follows from (9.7) since a symbol $\varphi$ constant on $[0,1]$ yields a rank one operator $Q_{\varphi}$.

We use methods from Sturm-Liouville theory, and begin by making some simplifications.

(i) Replacing $\varphi$ by a regularization $\varphi_{\varepsilon}$ such that $\varphi_{\varepsilon} \rightarrow \varphi$ in $L^{2}[0,1]$ and thus $Q_{\varphi_{\varepsilon}} \rightarrow Q_{\varphi}$ in $\boldsymbol{S}_{2}$ as $\varepsilon \rightarrow 0$, we see that we may assume $\varphi \in C^{1}[0,1]$.

(ii) Considering real and imaginary parts separately, we may assume that $\varphi$ is real, and thus $Q_{\varphi}$ self-adjoint.

(iii) Subtracting a constant times $\chi_{[0,1]}$, which yields a rank 1 operator, we may assume that $\varphi(1)=0$.

(iv) By homogeneity, we may assume that $\|\varphi\|_{B V}^{\prime}=\int_{0}^{1}\left|\varphi^{\prime}\right| \leq 1$ and show that then $\left\|Q_{\varphi}\right\|_{S_{1 / 2, \infty}} \leq C$.

(v) Using

$$
\begin{aligned}
\varphi(x) & =-\int_{x}^{1} \varphi^{\prime}(y) d y \\
& =-\int_{x}^{1} \max \left\{\varphi^{\prime}(y), 0\right\} d y-\int_{x}^{1} \min \left\{\varphi^{\prime}(y), 0\right\} d y \\
& =\varphi_{1}(x)-\varphi_{2}(x)
\end{aligned}
$$

and the corresponding decomposition $Q_{\varphi}=Q_{\varphi_{1}}-Q_{\varphi_{2}}$, we may also assume that $\varphi^{\prime} \leq 0$ and thus $\varphi \geq 0$. By Theorem 4.1, $Q_{\varphi}$ is then a positive operator.

(vi) Similarly, writing $\varphi=2 \varphi_{1}-\varphi_{2}$ with $\varphi_{2}=1-x$ and $\varphi_{1}=\left(\varphi+\varphi_{2}\right) / 2$, we may further assume that $\varphi^{\prime} \leq-1 / 2$ on $[0,1]$.

Let $\lambda>0$ and let, as above, $\left(G_{\lambda}, g_{\lambda}\right)$ be the solution to $(9.3)$ with $g_{\lambda}(0)=1$, $G_{\lambda}(0)=0$. Thus $\lambda$ is an eigenvalue if and only if (9.6) holds, i.e., by (iii), if and only if $g_{\lambda}(1)=0$.

Write $\omega=\lambda^{-1 / 2}$ and express $\left(g_{\lambda}, \omega G_{\lambda}\right)$ in polar coordinates

$$
\begin{aligned}
g_{\lambda}(x) & =R_{\omega}(x) \cos \Theta_{\omega}(x), \\
\omega G_{\lambda}(x) & =R_{\omega}(x) \sin \Theta_{\omega}(x),
\end{aligned}
$$

where $R_{\omega}(x)=\sqrt{g^{2}+\omega^{2} G^{2}}>0$ and $\Theta_{\omega}$ is continuous with $\Theta_{\omega}(0)=0$. Note that $\lambda$ is an eigenvalue if and only if $\cos \Theta_{\omega}(1)=0$, i.e. $\Theta_{\omega}(1)=n \pi+\pi / 2$ for some integer $n$.

Since $R_{\omega}(x)>0, R_{\omega}$ and $\Theta_{\omega}$ belong to $C^{1}[0,1]$, and (9.9) and (9.3) yield

$$
\lambda^{1 / 2} R_{\omega}^{2} \Theta_{\omega}^{\prime}=g G^{\prime}-G g^{\prime}=g^{2}-\lambda^{-1} \varphi^{\prime} G^{2}=R_{\omega}^{2}\left(\cos ^{2} \Theta_{\omega}-\varphi^{\prime} \sin ^{2} \Theta_{\omega}\right)
$$

and thus

$$
\Theta_{\omega}^{\prime}=\omega\left(\cos ^{2} \Theta_{\omega}-\varphi^{\prime} \sin ^{2} \Theta_{\omega}\right)
$$

In particular, since $\varphi^{\prime}<0$ by (vi), $\Theta_{\omega}^{\prime}>0$ and thus $\Theta_{\omega}(1)>0$. 
Now suppose $0<\omega<\nu$ and consider the corresponding functions $\Theta_{\omega}$ and $\Theta_{\nu}$. We claim that

$$
\Theta_{\omega}(x)<\Theta_{\nu}(x), \quad 0<x \leq 1 .
$$

Indeed, since $\Theta_{\omega}(0)=0=\Theta_{\nu}(0)$ and, by (9.10), $\Theta_{\omega}^{\prime}(0)=\omega<\nu=\Theta_{\nu}^{\prime}(0),(9.11)$ holds in $(0, \delta)$ for some $\delta>0$. Hence, if (9.11) fails, there exists some $x_{1} \in(0,1]$ such that $\Theta_{\omega}(x)<\Theta_{\nu}(x)$ for $0<x<x_{1}$ but $\Theta_{\omega}\left(x_{1}\right)=\Theta_{\nu}\left(x_{1}\right)$. This would imply $\Theta_{\omega}^{\prime}\left(x_{1}\right) \geq \Theta_{\nu}^{\prime}\left(x_{1}\right)$; on the other hand, then

$$
\cos ^{2} \Theta_{\omega}\left(x_{1}\right)-\varphi^{\prime} \sin ^{2} \Theta_{\omega}\left(x_{1}\right)=\cos ^{2} \Theta_{\nu}\left(x_{1}\right)-\varphi^{\prime} \sin ^{2} \Theta_{\nu}\left(x_{1}\right)>0,
$$

recalling (vi), and (9.10) would yield $\Theta_{\omega}^{\prime}\left(x_{1}\right)<\Theta_{\nu}^{\prime}\left(x_{1}\right)$, a contradiction.

From (9.11) follows in particular that the function $\omega \mapsto \Theta_{\omega}(1)$ is strictly increasing. Hence, there is for each integer $n \geq 0$ at most one value of $\omega, \omega_{n}$ say, such that $\Theta_{\omega_{n}}(1)=n \pi+\pi / 2$, and thus a corresponding eigenvalue $\lambda_{n}=\omega_{n}^{-2}$. (The solution to (9.4) depends continuously on $\omega$, with $\Theta_{0}(1)=0$ and $\Theta_{\omega}(1) \rightarrow \infty$ as $\omega \rightarrow \infty$, so $\omega_{n}$ exists for every $n \geq 1$, but we do not need that.) Integrating (9.10) we obtain by (iv)

$$
\Theta_{\omega}(1)=\int_{0}^{1} \Theta_{\omega}^{\prime}(x) d x \leq \omega \int_{0}^{1}\left(1+\left|\varphi^{\prime}(x)\right|\right) d x \leq 2 \omega
$$

and thus $2 \omega_{n} \geq \Theta_{\omega_{n}}(1)=n \pi+\pi / 2$, which yields

$$
\lambda_{n}=\omega_{n}^{-2} \leq 4 \pi^{-2}\left(n+\frac{1}{2}\right)^{-2} .
$$

Considering again functions on the whole half-line $\mathbb{R}_{+}$, we now can prove an endpoint result corresponding to Theorem 5.1.

Theorem 9.2. If $\varphi \in Y_{1 / 2}$, then $Q_{\varphi} \in \boldsymbol{S}_{1 / 2, \infty}$.

Proof. Define $A_{n}^{(k)}$ and $A^{(k)}$ as in (3.3) and (3.4), but now for all integers $k$. For $k \geq 1$, (3.7) holds for every $p$, and thus $\varphi \in Y_{1 / 2} \subset X_{1 / 2}$ implies that

$$
\sum_{k \geq 1}\left\|\mathcal{P}_{A^{(k)}} Q_{\varphi}\right\|_{\boldsymbol{S}_{1 / 2}}^{1 / 2} \leq \sum_{k \geq 1} \text { const } 2^{-p k / 2}<\infty .
$$

Moreover, by symmetry, $\left\|\mathcal{P}_{A^{(-k)}} Q_{\varphi}\right\|_{S_{1 / 2}}=\left\|\mathcal{P}_{A^{(k)}} Q_{\varphi}\right\|_{S_{1 / 2}}$ so

$$
\sum_{k \leq-1}\left\|\mathcal{P}_{A^{(k)}} Q_{\varphi}\right\|_{S_{1 / 2}}^{1 / 2}<\infty
$$

too. It follows that

$$
Q_{\varphi}-\mathcal{P}_{A^{(0)}} Q_{\varphi}=\sum_{k \neq 0} \mathcal{P}_{A^{(k)}} Q_{\varphi} \in \boldsymbol{S}_{1 / 2}
$$


Next, $\mathcal{P}_{A^{(0)}} Q_{\varphi}$ is the direct sum of $\mathcal{P}_{A_{n}^{(0)}} Q_{\varphi}, n \in \mathbb{Z}$, which act in the orthogonal spaces $L^{2}\left[2^{n}, 2^{n+1}\right]$. By translation invariance, Lemma 2.1 and Theorem 9.1, with $\varphi_{n}(x)=2^{n} \varphi\left(2^{n} x+2^{n}\right)$,

$$
\begin{aligned}
\left\|\mathcal{P}_{A_{n}^{(0)}} Q_{\varphi}\right\|_{S_{1 / 2, \infty}} & =\left\|Q_{\varphi}^{\left[2^{n}, 2^{n+1}\right]}\right\|_{S_{1 / 2, \infty}}=\left\|Q_{\varphi_{n}}^{[0,1]}\right\|_{S_{1 / 2, \infty}} \leq C\left\|\varphi_{n}\right\|_{B V[0,1]} \\
& \leq C^{\prime} 2^{n} \int_{2^{n}}^{\infty}|d \varphi| .
\end{aligned}
$$

By Theorem 5.3, we thus have

$$
\sum_{n \in \mathbb{Z}}\left(\left\|\mathcal{P}_{A_{n}^{(0)}} Q_{\varphi}\right\|_{S_{1 / 2, \infty}}\right)^{1 / 2}<\infty
$$

and it follows from Lemma 7.6 (or as in the proof of Lemma 9.5 below) that $\mathcal{P}_{A^{(0)}} Q_{\varphi} \in \boldsymbol{S}_{1 / 2, \infty}$. By $(9.12), Q_{\varphi} \in \boldsymbol{S}_{1 / 2, \infty}$ too.

Theorem 9.2 is the best possible; for any reasonably smooth $\varphi$, the singular numbers $s_{n}\left(Q_{\varphi}\right)$ decrease like $n^{-2}$ but not faster. More precisely, we have the following very precise result. Recall that a function in $Y_{1 / 2}$ has locally bounded variation and thus is a.e. differentiable.

Theorem 9.3. Let $\varphi \in Y_{1 / 2}$. Then

$$
n^{2} s_{n}\left(Q_{\varphi}\right) \rightarrow \pi^{-2}\left\|\varphi^{\prime}\right\|_{L^{1 / 2}}=\pi^{-2}\left(\int_{0}^{\infty}\left|\varphi^{\prime}(x)\right|^{1 / 2} d x\right)^{2}<\infty \quad \text { as } n \rightarrow \infty .
$$

Equivalently,

$$
\varepsilon^{1 / 2}\left|\left\{n: s_{n}\left(Q_{\varphi}\right)>\varepsilon\right\}\right| \rightarrow \pi^{-1} \int_{0}^{\infty}\left|\varphi^{\prime}(x)\right|^{1 / 2} d x<\infty \quad \text { as } \varepsilon \rightarrow 0 .
$$

In particular, $n^{2} s_{n}\left(Q_{\varphi}\right) \rightarrow 0$ as $n \rightarrow \infty$ if and only if $\varphi^{\prime}=0$ a.e.

Proof. Note first that by the Cauchy-Schwarz inequality and (5.1),

$$
\int_{0}^{\infty}\left|\varphi^{\prime}(x)\right|^{1 / 2} d x \leq \sum_{n \in \mathbb{Z}} 2^{n / 2}\left(\int_{2^{n}}^{2^{n+1}}\left|\varphi^{\prime}(x)\right| d x\right)^{1 / 2} \leq\|\varphi\|_{Y_{1 / 2}}^{1 / 2}<\infty .
$$

For smooth and positive symbols on a finite interval, (9.13) follows by standard Sturm-Liouville theory, see [LS, §1.2 with the transformation in $\S 1.1]$. Indeed, much more refined asymptotics of $s_{n}$ can be given [LS, Chapter 5].

We present here another proof that applies in the general case. We prove a sequence of lemmas. The first implies that (9.13) and (9.14) are equivalent.

Lemma 9.4. For any bounded operator $T$ on a Hilbert space,

$$
\limsup _{\varepsilon \rightarrow 0} \varepsilon^{1 / 2}\left|\left\{n: s_{n}>\varepsilon\right\}\right|=\left(\limsup _{n \rightarrow \infty}\left(n^{2} s_{n}\right)\right)^{1 / 2}
$$


and similarly with liminf instead of limsup on both sides.

Proof. If $\lim \sup \varepsilon^{1 / 2}\left|\left\{n: s_{n}>\varepsilon\right\}\right|<a$ for some $a>0$, then for all small $\varepsilon$, $\left|\left\{n: s_{n}>\varepsilon\right\}\right|<a \varepsilon^{-1 / 2}$. Taking $\varepsilon=a^{2}(n+1)^{-2}$, we see that for large $n, s_{n} \leq \varepsilon$, and thus $(n+1)^{2} s_{n} \leq a^{2}$, so $\limsup _{n \rightarrow \infty} n^{2} s_{n} \leq a^{2}$. The converse is similar, and the second part follows similarly by reversing the inequalities.

Lemma 9.5. If $T_{1}, \ldots, T_{N}$ are bounded operators on Hilbert spaces $H_{1}, \ldots, H_{N}$, then

$$
\left(\limsup _{n \rightarrow \infty} n^{2} s_{n}\left(T_{1} \oplus \cdots \oplus T_{N}\right)\right)^{1 / 2} \leq \sum_{k=1}^{N}\left(\limsup _{n \rightarrow \infty} n^{2} s_{n}\left(T_{k}\right)\right)^{1 / 2}
$$

and

$$
\left(\liminf _{n \rightarrow \infty} n^{2} s_{n}\left(T_{1} \oplus \cdots \oplus T_{N}\right)\right)^{1 / 2} \geq \sum_{k=1}^{N}\left(\liminf _{n \rightarrow \infty} n^{2} s_{n}\left(T_{k}\right)\right)^{1 / 2} .
$$

Proof. The singular numbers $s_{n}\left(T_{1} \oplus \cdots \oplus T_{N}\right)$ consist of all $s_{i}\left(T_{j}\right)$, rearranged into a single nonincreasing sequence. Hence,

$$
\left|\left\{n: s_{n}\left(T_{1} \oplus \cdots \oplus T_{N}\right)>\varepsilon\right\}\right|=\sum_{j=1}^{N}\left|\left\{n: s_{n}\left(T_{j}\right)>\varepsilon\right\}\right|
$$

and the result follows by Lemma 9.4.

For arbitrary sums we have the following estimate.

Lemma 9.6. If $T$ and $U$ are bounded operators in a Hilbert space, and $0<\delta<1$, then

$$
\begin{aligned}
& \limsup _{n \rightarrow \infty} n^{2} s_{n}(T+U) \leq(1-\delta)^{-2} \limsup _{n \rightarrow \infty} n^{2} s_{n}(T)+\delta^{-2} \limsup _{n \rightarrow \infty} n^{2} s_{n}(U), \\
& \liminf _{n \rightarrow \infty} n^{2} s_{n}(T+U) \geq(1-\delta)^{2} \liminf _{n \rightarrow \infty} n^{2} s_{n}(T)-\delta^{-2} \limsup _{n \rightarrow \infty} n^{2} s_{n}(U) .
\end{aligned}
$$

Proof. By $(2.1), s_{n}(T+U) \leq s_{[(1-\delta) n]}(T)+s_{[\delta n]}(U)$, and (9.16) follows, together with

$$
\liminf _{n \rightarrow \infty} n^{2} s_{n}(T+U) \leq(1-\delta)^{-2} \liminf _{n \rightarrow \infty} n^{2} s_{n}(T)+\delta^{-2} \limsup _{n \rightarrow \infty} n^{2} s_{n}(U) .
$$

Replacing here $T$ by $T+U$ and $U$ by $-U$, we obtain (9.17) by rearrangement.

Letting $\delta \rightarrow 0$ in (9.17) and (9.18), we obtain the following result by Fan [GK1].

Lemma 9.7. If $T$ and $U$ are bounded operators in a Hilbert space, $\lim _{n \rightarrow \infty} n^{2} s_{n}(T)$ exists and $n^{2} s_{n}(U) \rightarrow 0$ as $n \rightarrow \infty$, then $\lim _{n \rightarrow \infty} n^{2} s_{n}(T+U)=\lim _{n \rightarrow \infty} n^{2} s_{n}(T)$.

Lemma 9.8. The set of $\varphi \in Y_{1 / 2}$ such that (9.13) holds is a closed set. 
Proof. Suppose that $\varphi_{k} \rightarrow \varphi$ in $Y_{1 / 2}$ and that (9.13) holds for each $\varphi_{k}$. By Lemma 9.6 and Theorem 9.2, for every $k$ and $0<\delta<1$,

$$
\begin{aligned}
\limsup _{n \rightarrow \infty} n^{2} s_{n}\left(Q_{\varphi}\right) & \leq(1-\delta)^{-2} \limsup _{n \rightarrow \infty} n^{2} s_{n}\left(Q_{\varphi_{k}}\right)+\delta^{-2} \limsup _{n \rightarrow \infty} n^{2} s_{n}\left(Q_{\varphi-\varphi_{k}}\right) \\
& \leq(1-\delta)^{-2} \pi^{-2}\left\|\varphi_{k}^{\prime}\right\|_{L^{1 / 2}}+C \delta^{-2}\left\|\varphi-\varphi_{k}\right\|_{Y_{1 / 2}}
\end{aligned}
$$

and similarly

$$
\liminf _{n \rightarrow \infty} n^{2} s_{n}\left(Q_{\varphi}\right) \geq(1-\delta)^{2} \pi^{-2}\left\|\varphi_{k}^{\prime}\right\|_{L^{1 / 2}}-C \delta^{-2}\left\|\varphi-\varphi_{k}\right\|_{Y_{1 / 2}}
$$

Moreover, by (9.15), $\left\|\left(\varphi-\varphi_{k}\right)^{\prime}\right\|_{L^{1 / 2}} \leq\left\|\varphi-\varphi_{k}\right\|_{Y_{1 / 2}} \rightarrow 0$ as $k \rightarrow \infty$, and so $\left\|\varphi_{k}^{\prime}\right\|_{L^{1 / 2}} \rightarrow\left\|\varphi^{\prime}\right\|_{L^{1 / 2}}$. Letting first $k \rightarrow \infty$ and then $\delta \rightarrow 0$ in (9.19) and (9.20), we obtain (9.13).

Lemma 9.9. If $\varphi$ is linear on a finite interval $I$, then

$$
n^{2} s_{n}\left(Q_{\varphi}^{I}\right) \rightarrow \pi^{-2}\left(\int_{I}\left|\varphi^{\prime}(x)\right|^{1 / 2} d x\right)^{2} \quad \text { as } n \rightarrow \infty .
$$

Proof. Let $\varphi(x)=\alpha+\beta x$ with $\alpha, \beta$ complex numbers. Suppose first that $I=[0,1]$. By the example at the beginning of the section and homogeneity,

$$
s_{n}\left(Q_{-\beta+\beta x}^{I}\right)=|\beta| \pi^{-2}\left(n+\frac{1}{2}\right)^{-2}
$$

so $n^{2} s_{n}\left(Q_{-\beta+\beta x}^{I}\right) \rightarrow \pi^{-2}|\beta|$ as $n \rightarrow \infty$. Since $Q_{\alpha+\beta x}^{I}-Q_{-\beta+\beta x}^{I}=Q_{\alpha+\beta}^{I}$ is a rank one operator, Lemma 9.7 (or, more simply, $s_{n+1}\left(Q_{-\beta+\beta x}^{I}\right) \leq s_{n}\left(Q_{\alpha+\beta x}^{I}\right) \leq$ $\left.s_{n-1}\left(Q_{-\beta+\beta x}^{I}\right)\right)$ yields

$$
n^{2} s_{n}\left(Q_{\varphi}^{I}\right) \rightarrow \pi^{-2}|\beta|=\pi^{-2}\left(\int_{I}\left|\varphi^{\prime}(x)\right|^{1 / 2} d x\right)^{2} .
$$

If $I=[0, a]$, we have by Lemma 2.1 and $(9.21)$

$$
n^{2} s_{n}\left(Q_{\varphi}^{I}\right)=n^{2} s_{n}\left(Q_{\varphi_{a}}^{[0,1]}\right) \rightarrow \pi^{-2} a^{2}|\beta|=\pi^{-2}\left(\int_{I}\left|\varphi^{\prime}(x)\right|^{1 / 2} d x\right)^{2},
$$

and the general case follows by translation invariance.

\section{Completion of the proof of Theorem 9.3.}

Step 1. $\varphi$ is piecewise linear on $[0,1]$ and $\varphi=0$ on $(1, \infty)$. Let $0=t_{0}<t_{1}<$ $\cdots<t_{N}=1$ be such that $\varphi$ is linear on every $I_{i}=\left[t_{i-1}, t_{i}\right], i=1, \ldots, N$. Let $H_{i}=L^{2}\left(I_{i}\right)$, so $L^{2}[0,1]=H_{1} \oplus \cdots \oplus H_{N}$, and let $P_{i}: L^{2}[0,1] \rightarrow H_{i}$ denote the orthogonal projection.

Since each $P_{i} Q_{\varphi} P_{j}, i \neq j$, has rank $1, Q_{\varphi}-\sum_{i=1}^{N} P_{i} Q_{\varphi} P_{i}$ has finite rank and by Lemma 9.7 (or directly), it suffices to consider $\sum_{i=1}^{N} P_{i} Q_{\varphi} P_{i}=Q_{\varphi}^{I_{1}} \oplus \cdots \oplus Q_{\varphi}^{I_{N}}$. By 
Lemma 9.9,

$$
n^{2} s_{n}\left(Q_{\varphi}^{I_{i}}\right) \rightarrow \pi^{-2}\left(\int_{t_{i-1}}^{t_{i}}\left|\varphi^{\prime}(x)\right|^{1 / 2} d x\right)^{2}
$$

and thus Lemma 9.5 yields

$$
\left(\lim _{n \rightarrow \infty} n^{2} s_{n}\left(\sum_{i=1}^{N} P_{i} Q_{\varphi} P_{i}\right)\right)^{1 / 2}=\sum_{i=1}^{N}\left(\lim _{n \rightarrow \infty} n^{2} s_{n}\left(Q_{\varphi}^{I_{i}}\right)\right)^{1 / 2}=\pi^{-1} \int_{0}^{1}\left|\varphi^{\prime}(x)\right|^{1 / 2} d x
$$

which proves (9.13).

Step 2. $\varphi$ is absolutely continuous on $[0,1]$ and $\varphi=0$ on $(1, \infty)$. Approximate $\varphi^{\prime}$ by step functions $h_{n}$ such that $\left\|\varphi^{\prime}-h_{n}\right\|_{L^{1}[0,1]}<1 / n$, and let

$$
\psi_{n}(x)= \begin{cases}\varphi(0)+\int_{0}^{x} h_{n}(y) d y, & x \leq 1 \\ 0, & x>1\end{cases}
$$

Then (9.13) holds for each $\psi_{n}$ by Step 1 , and $\psi_{n} \rightarrow \varphi$ in $B V[0,1]$ and thus in $Y_{1 / 2}$, see Corollary 5.5, so (9.13) holds by Lemma 9.8.

Step 3. $\varphi$ has bounded variation on $[0,1], \varphi=0$ on $(1, \infty)$ and $\varphi$ is singular, i.e., $\varphi^{\prime}=0$ almost everywhere. We may assume that $\varphi$ is right-continuous. Then $\varphi(x)=\varphi(0)+\int_{0}^{x} d \mu$ for some singular complex measure $\mu$ supported on $[0,1]$. Given any $\varepsilon>0$, there thus exists a sequence of intervals $\left(I_{i}\right)_{1}^{\infty}$ in $[0,1]$ such that $\sum_{i=1}^{\infty}\left|I_{i}\right|<\varepsilon$ and $|\mu|\left([0,1] \backslash \bigcup_{i=1}^{\infty} I_{i}\right)=0$. Let $N$ be a positive integer such that $|\mu|\left([0,1] \backslash \bigcup_{i=1}^{N} I_{i}\right)<\varepsilon$

We may assume that each $I_{i}$ is closed, and by combining any two of $I_{1}, \ldots, I_{N}$ that overlap, we may assume that $I_{1}, \ldots, I_{N}$ are disjoint. The complement $[0,1] \backslash \bigcup_{i=1}^{N} I_{i}$ is also a finite disjoint union of intervals, say $\bigcup_{j=1}^{M} J_{j}$.

For each interval $I$, Theorem 9.1 and Lemma 2.1 yield

$$
\sup _{n \geq 1} n^{2} s_{n}\left(Q_{\varphi}^{I}\right) \leq C|I|\|\varphi\|_{B V(I)}^{\prime} \leq C|I||\mu|(I) .
$$

Moreover, as in Step 1 of the proof,

$$
Q_{\varphi}=Q_{\varphi}^{I_{1}} \oplus \cdots \oplus Q_{\varphi}^{I_{N}} \oplus Q_{40}^{J_{1}} \oplus \cdots \oplus Q_{\varphi}^{J_{M}}+R
$$


where $R$ has finite rank. Hence, by Lemma 9.5, (9.22) and the Cauchy-Schwarz inequality,

$$
\begin{aligned}
\left(\limsup _{n \rightarrow \infty} n^{2} s_{n}\left(Q_{\varphi}\right)\right)^{1 / 2} \leq & \sum_{i=1}^{N}\left(\limsup _{n \rightarrow \infty} n^{2} s_{n}\left(Q_{\varphi}^{I_{i}}\right)\right)^{1 / 2}+\sum_{j=1}^{M}\left(\limsup _{n \rightarrow \infty} n^{2} s_{n}\left(Q_{\varphi}^{J_{j}}\right)\right)^{1 / 2} \\
\leq & C \sum_{i=1}^{N}\left(\left|I_{i}\right||\mu|\left(I_{i}\right)\right)^{1 / 2}+C \sum_{j=1}^{M}\left(\left|J_{j}\right||\mu|\left(J_{j}\right)\right)^{1 / 2} \\
\leq & C\left(\sum_{i=1}^{N}\left|I_{i}\right|\right)^{1 / 2}\left(\sum_{i=1}^{N}|\mu|\left(I_{i}\right)\right)^{1 / 2} \\
& \quad+C\left(\sum_{j=1}^{M}\left|J_{j}\right|\right)^{1 / 2}\left(\sum_{j=1}^{M}|\mu|\left(J_{j}\right)\right)^{1 / 2} \\
\leq & C \varepsilon^{1 / 2}(|\mu|[0,1])^{1 / 2}+C \cdot 1 \cdot \varepsilon^{1 / 2} .
\end{aligned}
$$

The result $n^{2} s_{n}\left(Q_{\varphi}\right) \rightarrow 0$ follows by letting $\varepsilon \rightarrow 0$.

Step 4. $\varphi$ has bounded variation on $(0, a)$ and $\varphi=0$ on $(a, \infty)$ for some $a>0$. By Lemma 2.1, it suffices to consider the case $a=1$. We can decompose $\varphi=\varphi_{\mathrm{a}}+\varphi_{\mathrm{s}}$ on $[0,1]$, with $\varphi_{\mathrm{a}}$ absolutely continuous and $\varphi_{\mathrm{s}}$ singular; let $\varphi_{\mathrm{a}}=\varphi_{\mathrm{s}}=0$ on $(1, \infty)$. By Steps 2 and 3,

$$
n^{2} s_{n}\left(Q_{\varphi_{\mathrm{a}}}\right) \rightarrow \pi^{-2}\left\|\varphi_{\mathrm{a}}^{\prime}\right\|_{1 / 2}=\pi^{-2}\left\|\varphi^{\prime}\right\|_{1 / 2}
$$

and $n^{2} s_{n}\left(Q_{\varphi_{\mathrm{s}}}\right) \rightarrow 0$, and the result follows by Lemma 9.7.

Step 5. $\varphi \in Y_{1 / 2}$ is arbitrary. Define, for $N \geq 1$,

$$
\varphi_{N}(x)= \begin{cases}\varphi(1 / N)-\varphi(N), & 0<x \leq 2^{-N}, \\ \varphi(x)-\varphi(N), & 2^{-N}<x \leq 2^{N}, \\ 0, & 2^{N}<x .\end{cases}
$$

It is easily seen that each $\varphi_{N}$ has bounded variation and that $\left\|\varphi-\varphi_{N}\right\|_{Y_{1 / 2}} \rightarrow 0$ as $N \rightarrow \infty$, cf. (5.1). Thus the result follows by Step 4 and Lemma 9.8.

As corollaries, we obtain new proofs of some results from $\S 8$.

Corollary 9.10. If I is a finite interval and $\varphi$ has bounded variation on $I$, then

$$
n^{2} s_{n}\left(Q_{\varphi}^{I}\right) \rightarrow \pi^{-2}\left(\int_{I}\left|\varphi^{\prime}(x)\right|^{1 / 2} d x\right)^{2} \quad \text { as } n \rightarrow \infty .
$$

Proof. By translation invariance, we may assume $I=[0, a]$. Then, defining $\varphi=0$ outside $I$, we have $\varphi \in Y_{1 / 2}$ by Corollary 5.5 , and the result follows by Theorem 9.3.

Corollary 9.11. If $\varphi$ has locally bounded variation and $Q_{\varphi} \in \boldsymbol{S}_{1 / 2}$, then $\varphi^{\prime}=0$ a.e. 
Proof. If $0<a<b<\infty$, then $\varphi$ has bounded variation on $[a, b]$, and since

$$
n^{2} s_{n}\left(Q_{\varphi}^{[a, b]}\right) \leq n^{2} s_{n}\left(Q_{\varphi}\right) \rightarrow 0
$$

Corollary 9.10 yields $\int_{a}^{b}\left|\varphi^{\prime}\right|^{1 / 2}=0$. Hence, $\varphi^{\prime}=0$ a.e.

Corollary 9.12. If $\varphi$ is locally absolutely continuous and $Q_{\varphi} \in \boldsymbol{S}_{1 / 2}$, then $\varphi=0$.

Remark. More generally, in the last two corollaries, $\boldsymbol{S}_{1 / 2}$ can be replaced by any Schatten-Lorentz space $\boldsymbol{S}_{1 / 2, q}$ with $q<\infty$.

\section{More on $p=1$}

Although $\varphi \in X_{1}$ does not imply $Q_{\varphi}, Q_{\varphi}^{+} \in \boldsymbol{S}_{1}$, the corresponding weak results holds. There is, however, a striking difference between $Q_{\varphi}$ and $Q_{\varphi}^{+}$; as is shown in (ii) and (iii) below, for every $\varphi \in X_{1}$ not a.e. equal to $0, n s_{n} \rightarrow 0$ for $Q_{\varphi}$ but not for $Q_{\varphi}^{+}$. (Note that Theorem 6.5 implies that nothing can be said about the rate of convergence of $n s_{n}\left(Q_{\varphi}\right)$ to 0 . In particular, if $q<\infty$, then $\varphi \in X_{1}$ does not imply that $Q_{\varphi} \in \boldsymbol{S}_{1, q}$.)

Theorem 10.1. If $\varphi \in X_{1}$ then the following hold:

(i) $Q_{\varphi}, Q_{\varphi}^{+}, Q_{\varphi}^{-} \in \boldsymbol{S}_{1, \infty}$.

(ii) $n s_{n}\left(Q_{\varphi}\right) \rightarrow 0$ as $n \rightarrow \infty$.

(iii) $n s_{n}\left(Q_{\varphi}^{+}\right)=n s_{n}\left(Q_{\varphi}^{-}\right) \rightarrow \pi^{-1} \int_{0}^{\infty}|\varphi(x)| d x$ as $n \rightarrow \infty$.

Proof. Since $X_{1} \subset X_{\infty}, Q_{\varphi}^{+}$is bounded by Theorem 3.1. By Theorem 4.2,

$$
\left(Q_{\varphi}^{+}\right)^{*} Q_{\varphi}^{+}=Q_{\Phi}
$$

where $\Phi(x)=\int_{x}^{\infty}|\varphi(y)|^{2} d y$. By $(1.5), x^{1 / 2} \Phi(x)^{1 / 2} \in L^{1}(d x / x)$, so $\Phi \in Y_{1 / 2}$ by Theorem 5.3, and hence, $Q_{\Phi} \in \boldsymbol{S}_{1 / 2, \infty}$ by Theorem 9.2. Consequently, $Q_{\varphi}^{+} \in \boldsymbol{S}_{1, \infty}$. The same holds for $Q_{\varphi}^{-}=\left(Q_{\varphi}^{+}\right)^{*}$ and $Q_{\varphi}=Q_{\varphi}^{+}+Q_{\varphi}^{-}$.

Moreover, $s_{n}\left(Q_{\Phi}\right)=s_{n}\left(Q_{\varphi}^{+}\right)^{2}$, and thus (iii) follows from Theorem 9.3 applied to $\Phi$.

For (ii), we observe that $\varphi \mapsto Q_{\varphi}$ thus is a bounded linear map $X_{1} \rightarrow \boldsymbol{S}_{1, \infty}$, and that the set of $C^{1}$ functions with compact support is dense in $X_{1}$ and mapped (by Theorem 9.2) into the closed subspace $\boldsymbol{S}_{1, \infty}^{0}=\left\{T \in \boldsymbol{S}_{1, \infty}: n s_{n}(T) \rightarrow 0\right.$ as $\left.n \rightarrow \infty\right\}$ of $\boldsymbol{S}_{1, \infty}$. Hence, $Q_{\varphi} \in \boldsymbol{S}_{1, \infty}^{0}$ for every $\varphi \in X_{1}$. 
Remark. Note that (i) and (iii) were earlier obtained in [EEH] for a more general class of operators. Moreover, in [EEH] the authors also consider the same operators on $L^{p}\left(\mathbb{R}_{+}\right)$and obtain similar results for approximation numbers. See also related results in $[\mathrm{NaS}]$. We also mention here [GK1, Remark IV.8.3] and [GK2, Theorem III.2.4], where similar asymptotic formulas are given for abstract Volterra operators with trace class imaginary parts.

Remark. For the related operators $\mathbf{Q}_{\mu}^{+}$, we similarly obtain that if $x \mu(x, \infty) \in$ $L^{1 / 2}(d x / x)$, then $\mathbf{Q}_{\mu}^{+} \in \boldsymbol{S}_{1, \infty}$, and

$$
n s_{n}\left(\mathbf{Q}_{\mu}^{+}\right) \rightarrow \pi^{-1} \int_{0}^{\infty}\left(\frac{d \mu}{d x}\right)^{1 / 2} d x \quad \text { as } n \rightarrow \infty,
$$

where $\frac{d \mu}{d x}$ is the Radon-Nikodym derivative of the absolutely continuous component of $\mu$. In particular, for such $\mu, n s_{n}\left(\mathbf{Q}_{\mu}^{+}\right) \rightarrow 0$ if and only if $\mu$ is singular.

We saw earlier that $\varphi \in X_{1}$ is not enough to insure that $Q_{\varphi}$ is in the trace class. Furthermore the previous theorem shows that if $\varphi \in X_{1}$ then neither $Q_{\varphi}^{+}$ nor $Q_{\varphi}^{-}$will be in the trace class. However, the combination of size and regularity results for singular numbers given in the previous theorem does insure that these operators have a well defined Dixmier trace. Because of the recent interest in the Dixmier trace we digress briefly to record this observation. For more about the Dixmier trace and its uses we refer to IV.2. $\beta$ of $[\mathrm{C}]$.

Let $\ell^{\infty}$ be the space of bounded sequences indexed by non-negative integers and let $c_{1}$ be the closed subspace consisting of sequences $\left\{a_{n}\right\}$ for which $\lim _{n} a_{n}$ exists. It follows from the Hahn-Banach theorem that the functional $\lim (\cdot)$ which is defined on $c_{1}$ has a positive continuous extension, $\lim _{\omega}(\cdot)$, to all of $\ell^{\infty}$. By saying $\lim _{\omega}(\cdot)$ is positive we mean that if $a_{n} \geq 0$ for $n=0,1,2, \ldots$ then $\lim _{\omega}\left(\left\{a_{n}\right\}\right) \geq 0$. This extension is not unique and we are using the subscript $\omega$ to denote the particular choice. It was noted by Dixmier in $[D]$ that $\lim _{\omega}(\cdot)$ can also be selected to have the following scaling property:

$$
\lim _{\omega}\left(a_{0}, a_{0}, a_{1}, a_{1}, a_{2}, a_{2}, \ldots\right)=\lim _{\omega}\left(a_{0}, a_{1}, a_{2}, \ldots\right) .
$$

A simple proof is in $[\mathrm{C}]$. (Although the scaling is important for the general theory it has no role in our discussion.)

Consider now the operator ideal $\boldsymbol{S}_{\Omega} \supset \boldsymbol{S}_{1, \infty}$ that consists of the operators $T$ on Hilbert space such that

$$
\|T\|_{S_{\Omega}} \stackrel{\text { def }}{=} \sup _{n \geq 0} \frac{\sum_{k=0}^{n} s_{k}(T)}{\sum_{k=0}^{n} \frac{1}{k+1}}<\infty .
$$


Suppose that $T \in \boldsymbol{S}_{\Omega}$. For a fixed choice of $\lim _{\omega}(\cdot)$ we define a Dixmier trace, $\operatorname{trace}_{\omega}(\cdot)$, as follows. For positive $T \in \boldsymbol{S}_{\Omega}$ set

$$
\operatorname{trace}_{\omega}(T)=\lim _{\omega}\left(\left\{\frac{1}{\log (n+2)} \sum_{k=0}^{n} \lambda_{k}(T)\right\}\right) .
$$

Here the $\lambda_{k}$ are the (necessarily non-negative) eigenvalues of the positive operator $\mathrm{T}$ arranged in decreasing order. Although perhaps not obvious at first glance, it is not difficult to see that, in fact, if $T_{1}$ and $T_{2}$ are two positive operators in $\boldsymbol{S}_{\Omega}$ then $\operatorname{trace}_{\omega}\left(T_{1}+T_{2}\right)=\operatorname{trace}_{\omega}\left(T_{1}\right)+\operatorname{trace}_{\omega}\left(T_{2}\right)$. A proof of this is also in [C]. Using this fact, the functional trace $\omega_{\omega}(\cdot)$ can be extended uniquely by linearity to all of $T \in \boldsymbol{S}_{\Omega}$. For $T \in \boldsymbol{S}_{\Omega}$ the value of $\operatorname{trace}_{\omega}(T)$ need not be independent of $\omega$. However, there are certain operators for which trace $\omega(T)$ is independent of $\omega$. Such operators are defined to be measurable. In this case we will write $\operatorname{trace}_{\mathrm{D}}(T)$ for this common value and refer to it as the Dixmier trace of $T$.

\section{Corollary 10.2.}

(i) If $\varphi \in X_{1}$, then the operators $\left|Q_{\varphi}^{+}\right|$and $\left|Q_{\varphi}^{-}\right|$are measurable and

$$
\operatorname{trace}_{\mathrm{D}}\left(\left|Q_{\varphi}^{+}\right|\right)=\operatorname{trace}_{\mathrm{D}}\left(\left|Q_{\varphi}^{-}\right|\right)=\frac{1}{\pi} \int_{0}^{\infty}|\varphi(x)| d x .
$$

(ii) If $\varphi \in Y_{1 / 2}$, then $\left|Q_{\varphi}\right|^{1 / 2}$ is measurable and

$$
\operatorname{trace}_{\mathrm{D}}\left(\left|Q_{\varphi}\right|^{1 / 2}\right)=\frac{1}{\pi} \int_{0}^{\infty}\left|\varphi^{\prime}(x)\right|^{1 / 2} d x .
$$

(iii) If $\varphi \in X_{1}$, then $Q_{\varphi}$ is measurable and

$$
\operatorname{trace}_{\mathrm{D}}\left(Q_{\varphi}\right)=0 \text {. }
$$

(iv) If $\varphi \in X_{1}$, then $Q_{\varphi}^{+}$and $Q_{\varphi}^{-}$are measurable and

$$
\operatorname{trace}_{\mathrm{D}}\left(Q_{\varphi}^{+}\right)=\operatorname{trace}_{\mathrm{D}}\left(Q_{\varphi}^{-}\right)=0 .
$$

Proof. We start with (i). From the very definitions $s_{n}\left(\left|Q_{\varphi}^{+}\right|\right)=s_{n}\left(Q_{\varphi}^{+}\right)$and hence, the previous theorem gives the asymptotic behavior of $\left\{s_{n}\left(\left|Q_{\varphi}^{+}\right|\right)\right\}_{n \geq 0}$. Those asymptotics, together with the fact that $\left|Q_{\varphi}^{+}\right|$is a positive operator, insure that $\left|Q_{\varphi}^{+}\right|$is measurable and has the indicated Dixmier trace. A similar argument applies to $\left|Q_{\varphi}^{-}\right|$and, after noting that $s_{n}\left(\left|Q_{\varphi}\right|^{1 / 2}\right)=s_{n}\left(Q_{\varphi}\right)^{1 / 2}$ and taking note of Theorem 9.3, to part (ii).

We now consider (iii). By Theorem 10.1, we have $\lim _{n \rightarrow \infty} n s_{n}\left(Q_{\varphi}\right)=0$. Also $s_{n}\left(Q_{\varphi}\right)=s_{n}\left(Q_{\varphi}^{*}\right)$. Thus by $(2.1), \lim _{n \rightarrow \infty} n s_{n}\left(\left(Q_{\varphi}+Q_{\varphi}^{*}\right) / 2\right) \stackrel{n \rightarrow \infty}{=} 0$. We now use the spectral projection to write $\frac{1}{2}\left(Q_{\varphi}+Q_{\varphi}^{*}\right)$ as a difference of two positive operators 
$\frac{1}{2}\left(Q_{\varphi}+Q_{\varphi}^{*}\right)_{ \pm}$and note that we will have

$$
\lim _{n \rightarrow \infty} n s_{n}\left(\frac{1}{2}\left(Q_{\varphi}+Q_{\varphi}^{*}\right)_{ \pm}\right)=0 .
$$

Arguing similarly with the skew-adjoint part of $Q_{\varphi}$, we realize $Q_{\varphi}$ as a linear combination of four positive operators each of which have singular numbers which tend to zero more rapidly than $n^{-1}$. Those positive operators are certainly measurable and have Dixmier trace zero. The result we want now follows by the linearity of $\operatorname{trace}_{\mathrm{D}}(\cdot)$.

For (iv) we first pick and fix a choice $\operatorname{trace}_{\omega}(\cdot)$. Assume for the moment that $\varphi$ is real, supported in $[0,1]$ and in $L^{2} . R_{\varphi} \stackrel{\text { def }}{=} Q_{\varphi}^{+}-Q_{\varphi}^{+*}=Q_{\varphi}^{+}-Q_{\varphi}^{-}$has real anti-symmetric kernel. Thus it is normal and its eigenvalues are imaginary and symmetric. Hence, $\mathrm{i} R_{\varphi}$ is symmetric and its positive and negative parts, $\left(\mathrm{i} R_{\varphi}\right)_{ \pm}$ are unitarily equivalent. Thus

$$
\operatorname{trace}_{\omega}\left(R_{\varphi}\right)=-\mathrm{i} \operatorname{trace}_{\omega}\left(\left(\mathrm{i} R_{\varphi}\right)_{+}\right)+\mathrm{i} \operatorname{trace}_{\omega}\left(\left(\mathrm{i} R_{\varphi}\right)_{-}\right)=0 .
$$

Taking note of the fact that $\lim _{\omega}(\cdot)$ is continuous on $\ell^{\infty}$ and of the norm estimates implicit in the previous theorem we see that we can extend this result by linearity and continuity and conclude that $\operatorname{trace}_{\omega}\left(R_{\varphi}\right)=0$ for all $\varphi \in X_{1}$. Now we use the fact that $\omega$ was arbitrary to conclude $\operatorname{trace}_{\mathrm{D}}\left(R_{\varphi}\right)=0$. By linearity this result together with the result in (iii) yields (iv).

For a function $\varphi$ defined on a finite or infinite interval $I$, we define the standard and $L^{p}$ moduli of continuity by

$$
\begin{aligned}
& \omega_{\varphi}^{(\infty)}(h ; I) \stackrel{\text { def }}{=} \sup \{|\varphi(x)-\varphi(y)|: x, y \in I,|x-y| \leq h\}, \\
& \omega_{\varphi}^{(p)}(h ; I) \stackrel{\text { def }}{=} \sup _{0 \leq s \leq h}\left(\int_{I \cap(I-s)}|\varphi(x+s)-\varphi(x)|^{p} d x\right)^{1 / p}, \quad 1 \leq p<\infty,
\end{aligned}
$$

where $0<h \leq|I|$ and $I-s=\{x-s: x \in I\}=\{x: x+s \in I\}$. It follows easily from Minkowski's inequality that

$$
\omega_{\varphi}^{(p)}(h ; I) \leq 2 \omega_{\varphi}^{(p)}(h / 2 ; I), \quad 1 \leq p \leq \infty .
$$

Note further that for a finite interval $I$,

$$
\omega_{\varphi}^{(p)}(h ; I) \leq|I|^{1 / p-1 / q} \omega_{\varphi}^{(q)}(h ; I), \quad p<q \leq \infty .
$$

We often omit $I$ from the notation.

An alternative $L^{p}$ modulus of continuity is defined by

$$
\widetilde{\omega}_{\varphi}^{(p)}(h ; I) \stackrel{\text { def }}{=}\left((2 h)^{-1} \iint_{\substack{x, y \in I \\|x-y|<h}}|\varphi(x)-\varphi(y)|^{p} d x d y\right)^{1 / p} .
$$


This is equivalent to $\omega_{\varphi}^{(p)}(h ; I)$ defined above by the following lemma, which probably is well-known to some experts.

Lemma 10.3. Let $1 \leq p<\infty$. Then, with $C_{p}$ depending on $p$ only,

$$
\widetilde{\omega}_{\varphi}^{(p)}(h ; I) \leq \omega_{\varphi}^{(p)}(h ; I) \leq C_{p} \widetilde{\omega}_{\varphi}^{(p)}(h ; I) .
$$

Proof. The left hand inequality follows by

$$
\begin{aligned}
\left(\widetilde{\omega}_{\varphi}^{(p)}(h ; I)\right)^{p} & =\frac{1}{h} \iint_{\substack{x, y \in I \\
0<y-x<h}}|\varphi(y)-\varphi(x)|^{p} d x d y \\
& =\frac{1}{h} \int_{0}^{h} \int_{x \in I \cap(I-s)}|\varphi(x+s)-\varphi(x)|^{p} d x d y \leq\left(\omega_{\varphi}^{(p)}(h ; I)\right)^{p} .
\end{aligned}
$$

For the converse, we assume for convenience that $I=[0,1]$. The result then follows for every finite $I$ by a linear change of variables, and for infinite $I$ by considering $I \cap[-n, n]$ and letting $n \rightarrow \infty$. Thus $I=[0,1]$ and $I \cap(I-s)=[0,1-s]$.

Let $\varphi_{s}(x)=\varphi(x+s)$. Assume first that $h \leq 1 / 2$. Then, for $0 \leq s, t \leq h$, by Minkowski's inequality,

$$
\left\|\varphi-\varphi_{s}\right\|_{L^{p}[0,1 / 2]}^{p} \leq C_{p}\left\|\varphi-\varphi_{t}\right\|_{L^{p}[0,1 / 2]}^{p}+C_{p}\left\|\varphi_{s}-\varphi_{t}\right\|_{L^{p}[0,1 / 2]}^{p} .
$$

Averaging over $t \in[0, h]$ we find

$$
\begin{aligned}
\left\|\varphi-\varphi_{s}\right\|_{L^{p}[0,1 / 2]}^{p} & \leq \frac{C_{p}}{h} \int_{0}^{h} \int_{0}^{1 / 2}\left(|\varphi(x)-\varphi(x+t)|^{p}+|\varphi(x+s)-\varphi(x+t)|^{p}\right) d x d t \\
& \leq \frac{C_{p}}{h} \iint_{\substack{x, y \in[0,1] \\
0<y-x<h}}|\varphi(y)-\varphi(x)|^{p} d x d y=C_{p}\left(\widetilde{\omega}_{\varphi}^{(p)}(h ;[0,1])\right)^{p} .
\end{aligned}
$$

A similar argument, now taking $s-h \leq t \leq s$, yields the same estimate for $\left\|\varphi-\varphi_{s}\right\|_{L^{p}[1 / 2-s, 1-s]}^{p}$, and summing we find

$$
\left\|\varphi-\varphi_{s}\right\|_{L^{p}[0,1-s]} \leq C_{p} \widetilde{\omega}_{\varphi}^{(p)}(h ;[0,1])
$$

for every $0 \leq s \leq h$, which proves the result for $h \leq 1 / 2$.

If $1 / 2<h \leq 1$, the result follows from the case $\bar{h} \leq 1 / 2$ and (10.3).

For simplicity, we state the following lemma for $I=[0,1]$ only.

Lemma 10.4. Let $1 \leq p<\infty$. If $\varphi \in L^{p}[0,1]$ and $0<t \leq 1$, there exists a decomposition $\varphi=\varphi_{0}+\varphi_{1}$ with

$$
\left\|\varphi_{0}\right\|_{L^{p}[0,1]} \leq C_{p} \omega_{\varphi}^{(p)}(t) \quad \text { and } \quad\left\|\varphi_{1}\right\|_{B V[0,1]}^{\prime} \leq C_{p} t^{-1} \omega_{\varphi}^{(p)}(t) .
$$


In other words, the Peetre $K$-functional (see [BL]), can be estimated by

$$
K\left(t, \varphi ; L^{p}[0,1], B V^{\prime}[0,1]\right) \leq C_{p} \omega_{\varphi}^{(p)}(t), \quad 0<t \leq 1 .
$$

Proof. Take $\varphi_{1}(x)=\frac{1}{t} \int_{(1-t) x}^{(1-t) x+t} \varphi(y) d y$ and $\varphi_{0}=\varphi-\varphi_{1}$. Then $\varphi_{1}$ is absolutely continuous, and thus

$$
\begin{aligned}
\left\|\varphi_{1}\right\|_{B V}^{\prime} & =\int_{0}^{1}\left|\varphi_{1}^{\prime}(x)\right| d x=\frac{1-t}{t} \int_{0}^{1}|\varphi((1-t) x+t)-\varphi((1-t) x)| d x \\
& =\frac{1}{t} \int_{0}^{1-t}|\varphi(y+t)-\varphi(y)| d y \\
& \leq \frac{1}{t}\left(\int_{0}^{1-t}|\varphi(y+t)-\varphi(y)|^{p} d y\right)^{1 / p} \leq \frac{1}{t} \omega_{\varphi}^{(p)}(t) .
\end{aligned}
$$

Moreover, using Hölder's inequality again,

$$
\left|\varphi_{0}(x)\right|^{p}=\left|\frac{1}{t} \int_{(1-t) x}^{(1-t) x+t}(\varphi(x)-\varphi(y)) d y\right|^{p} \leq \frac{1}{t} \int_{(1-t) x}^{(1-t) x+t}|\varphi(x)-\varphi(y)|^{p} d y
$$

and thus

$$
\int_{0}^{1}\left|\varphi_{0}(x)\right|^{p} \leq \frac{1}{t} \iint_{\substack{x, y \in[0,1] \\|y-x|<t}}|\varphi(y)-\varphi(x)|^{p} d x d y=2\left(\omega_{\varphi}^{(p)}(t)\right)^{p} .
$$

Theorem 10.5. If $I$ is a finite interval and $\varphi \in L^{2}(I)$, then

$$
s_{n}\left(Q_{\varphi}^{I}\right) \leq C \frac{|I|^{1 / 2}}{n} \omega_{\varphi}^{(2)}\left(\frac{|I|}{n}\right) \leq C \frac{|I|}{n} \omega_{\varphi}^{(\infty)}\left(\frac{|I|}{n}\right), \quad n \geq 1 .
$$

Proof. By a linear change of variables, we may assume that $I=[0,1]$, cf. Lemma 2.1. Then, using the decomposition given by Lemma 10.4 with $t=1 / n$, (2.1), Theorem 10.1 and Theorem 9.1, we find, for $n \geq 1$,

$s_{2 n-1}\left(Q_{\varphi}\right) \leq s_{n-1}\left(Q_{\varphi_{0}}\right)+s_{n}\left(Q_{\varphi_{1}}\right) \leq C n^{-1}\left\|\varphi_{0}\right\|_{L^{2}}+C n^{-2}\left\|\varphi_{1}\right\|_{B V}^{\prime} \leq C n^{-1} \omega_{\varphi}^{(2)}(1 / n)$, and the result follows, using (10.3) and (10.4).

In particular, we see that a Dini condition implies $Q_{\varphi} \in \boldsymbol{S}_{1}$.

Corollary 10.6. If $\varphi \in L^{2}[0,1]$ is such that $\int_{0}^{1} \omega_{\varphi}^{(2)}(t) \frac{d t}{t}<\infty$, in particular if $\int_{0}^{1} \omega_{\varphi}^{(\infty)}(t) \frac{d t}{t}<\infty$, then $Q_{\varphi}^{[0,1]} \in \boldsymbol{S}_{1}$.

Proof. Theorem 3.1 shows that $Q_{\varphi}$ is bounded, and Theorem 10.5 yields

$$
\sum_{n=2}^{\infty} s_{n}\left(Q_{\varphi}\right) \leq C \sum_{n=2}^{\infty} \frac{1}{n} \omega_{\varphi}^{(2)}\left(\frac{1}{n}\right) \leq C \int_{0}^{1} \omega_{\varphi}^{(\infty)}(t) \frac{d t}{t} .
$$


By a simple change of variables, Corollary 10.6 applies to other finite intervals too. Moreover, for functions $\varphi$ on $\mathbb{R}_{+}$, we have the following corresponding sufficient conditions for $Q_{\varphi} \in \boldsymbol{S}_{1}$.

Theorem 10.7. If $\varphi \in X_{1}$ and

$$
\sum_{n=-\infty}^{\infty} 2^{n / 2} \int_{0}^{2^{n}} \omega_{\varphi}^{(2)}\left(t ;\left[2^{n}, 2^{n+1}\right]\right) \frac{d t}{t}<\infty
$$

then $Q_{\varphi} \in \boldsymbol{S}_{1}$.

Proof. Let $I_{n}=\left[2^{n}, 2^{n+1}\right]$. Then Theorem 10.5 yields

$$
\begin{aligned}
\left\|Q_{\varphi}^{I_{n}}\right\|_{\boldsymbol{S}_{1}} & =\sum_{k=0}^{\infty} s_{k}\left(Q_{\varphi}^{I_{n}}\right) \leq 2\left\|Q_{\varphi}^{I_{n}}\right\|_{S_{2}}+C\left|I_{n}\right|^{1 / 2} \sum_{k=2}^{\infty} \frac{1}{k} \omega_{\varphi}^{(2)}\left(\frac{2^{n}}{k} ; I_{n}\right) \\
& \leq C 2^{n / 2}\|\varphi\|_{L^{2}\left(I_{n}\right)}+C 2^{n / 2} \int_{0}^{2^{n}} \omega_{\varphi}^{(2)}\left(t ; I_{n}\right) \frac{d t}{t}
\end{aligned}
$$

and the result follows by Theorem 6.4 and (1.3).

Corollary 10.8. If $\varphi \in X_{1}$ and

$$
\sum_{n=-\infty}^{\infty} 2^{n} \int_{0}^{2^{n}} \omega_{\varphi}^{(\infty)}\left(t ;\left[2^{n}, 2^{n+1}\right]\right) \frac{d t}{t}<\infty
$$

then $Q_{\varphi} \in \boldsymbol{S}_{1}$.

Note that for the functions $\varphi_{N}$ considered in Theorem 6.5, the estimate of the singular numbers in Theorem 10.5 is sharp (within a constant factor) and the estimates of the $\boldsymbol{S}_{1}$ norm implicit in Corollary 10.6, Theorem 10.7 and Corollary 10.8 are of the right order.

We do not know whether the condition in Theorem 10.7 is necessary, but we will give a related necessary condition using the $L^{1}$ modulus of continuity in $\S 15$.

We have in these applications of Theorem 10.5 considered $\boldsymbol{S}_{1}$ only, but the same arguments apply to $\boldsymbol{S}_{p}$ for other $p$ too. In particular, Theorem 10.7 extends as follows (see the remark after Theorem 6.4).

Theorem 10.9. Let $1 / 2<p \leq 1$. If $\varphi \in X_{p}$ and

$$
\sum_{n=-\infty}^{\infty} 2^{n(1-p / 2)} \int_{0}^{2^{n}}\left(\omega_{\varphi}^{(2)}\left(t ;\left[2^{n}, 2^{n+1}\right]\right)\right)^{p} t^{p-2} d t<\infty
$$

then $Q_{\varphi} \in \boldsymbol{S}_{p}$.

Note also the following immediate consequence of Theorem 10.5. 
Corollary 10.10. If I is a finite interval and $\varphi$ satisfies a Hölder (Lipschitz) condition $|f(x)-f(y)| \leq C|x-y|^{\alpha}$ for $x, y \in I$, where $0<\alpha \leq 1$, then $Q_{\varphi} \in \boldsymbol{S}_{1 /(1+\alpha), \infty}$ and thus $Q_{\varphi} \in \boldsymbol{S}_{p}$ for every $p>1 /(1+\alpha)$.

\section{Averaging projection}

In this section we study properties of the averaging projection onto the set of operators of the form $Q_{\psi}$. Let us first define the averaging projection on $\boldsymbol{S}_{2}$. Let $T$ be an operator on $L^{2}\left(\mathbb{R}_{+}\right)$of class $\boldsymbol{S}_{2}$ with kernel function $k=k_{T} \in L^{2}\left(\left(\mathbb{R}_{+}\right)^{2}\right)$. We define the function $\varphi$ on $\mathbb{R}_{+}$by

$$
\varphi(x)=\frac{1}{2 x}\left(\int_{0}^{x} k(x, t) d t+\int_{0}^{x} k(s, x) d s\right), \quad x>0 .
$$

We define the averaging projection $\mathcal{Q}$ on $\boldsymbol{S}_{2}$ by

$$
\mathcal{Q} T \stackrel{\text { def }}{=} Q_{\varphi} \text {. }
$$

It is not hard to see that if $Q_{\psi} \in \boldsymbol{S}_{2}$, then $\mathcal{Q} Q_{\psi}=Q_{\psi}$. It is also easy to see that $\|\mathcal{Q} T\|_{\boldsymbol{S}_{2}} \leq\|T\|_{\boldsymbol{S}_{2}}$ for any $T \in \boldsymbol{S}_{2}$, and so $\mathcal{Q}$ is the orthogonal projection of $\boldsymbol{S}_{2}$ onto the set of operators of the form $Q_{\psi}$.

We show in this section that $\mathcal{Q}$ is a bounded linear operator on $\boldsymbol{S}_{p}$ for $1<p \leq 2$. This allows us to define by duality the projection $\mathcal{Q}$ on the classes $\boldsymbol{S}_{p}$ for $2 \leq p<\infty$. We also show that $\mathcal{Q}$ is unbounded on $\boldsymbol{S}_{1}$ but it has weak type $(1,1)$, i.e., $s_{n}(\mathcal{Q} T)(1+n) \leq$ const $\|T\|_{\boldsymbol{S}_{1}}$. Finally, we use this result to show that $\mathcal{Q}$ maps the Matsaev ideal into the set of compact operators.

Theorem 11.1. Let $1<p \leq 2$. Then $\mathcal{Q}$ is a bounded projection on $\boldsymbol{S}_{p}$.

Proof. Let $T$ be an integral operator in $\boldsymbol{S}_{p}$ with kernel function $k$ and let $\varphi$ be defined by (11.1). We have to show that $\varphi \in X_{p}$ (see the definition in the Introduction). We can identify in a natural way the dual space $X_{p}^{*}$ with the space $Z_{p^{\prime}}$ of functions $f$ on $\mathbb{R}_{+}$such that

$$
\sum_{n \in \mathbb{Z}} 2^{-n p^{\prime} / 2}\left(\int_{2^{n}}^{2^{n+1}}|f(x)|^{2} d x\right)^{p^{\prime} / 2}<\infty
$$

with respect to the pairing

$$
(\varphi, f)=\int_{0}^{\infty} \varphi(x) f(x) d x
$$

Here $p^{\prime}=p /(p-1)$. Suppose that $f$ is a function on $(0, \infty)$. Define the function $\psi$ on $\mathbb{R}_{+}$by $\psi(x)=\frac{f(x)}{2 x}, x>0$. It is straightforward to see from the definition of 
the $X_{p}$ spaces that $f \in X_{p}^{*}$ if and only if $\psi \in X_{p^{\prime}}$ and the norm of $f$ in $X_{p}^{*}$ and the norm of $\psi$ in $X_{p^{\prime}}$ are equivalent. It is also easy to see that for $1<p<\infty$ the space $X_{p}$ is reflexive.

Let us show that if $f$ is a bounded function in $X_{p}^{*}$ with compact support in $(0, \infty)$, then

$$
(\varphi, f)=\operatorname{trace} T Q_{\psi}
$$

We have

$$
\begin{aligned}
\operatorname{trace} T Q_{\psi} & =\iint_{\mathbb{R}_{+}^{2}} k(x, y) \psi(\max \{x, y\}) d x d y \\
& =\int_{0}^{\infty} \psi(x)\left(\int_{0}^{x} k(x, t) d t+\int_{0}^{x} k(s, x) d s\right) d x \\
& =\int_{0}^{\infty} 2 x \psi(x) \varphi(x) d x=\int_{0}^{\infty} \varphi(x) f(x) d x .
\end{aligned}
$$

It follows that

$$
\begin{aligned}
\sup \left\{|(\varphi, f)|: f \in X_{p}^{*},\|f\|_{X_{p}^{*}} \leq 1\right\} & \leq \text { const }\|T\|_{S_{p}} \sup \left\{\left\|Q_{\psi}\right\|_{S_{p^{\prime}}}:\|\psi\|_{X_{p^{\prime}}} \leq 1\right\} \\
& \leq \text { const }\|T\|_{\boldsymbol{S}_{p}}
\end{aligned}
$$

by Theorem 3.3. It follows that $\varphi \in X_{p}$, and again by Theorem $3.3, \mathcal{Q} T \in \boldsymbol{S}_{p}$.

Theorem 11.1 allows us to define for $1<p<2$ the adjoint operator $\mathcal{Q}^{*}$ on $\boldsymbol{S}_{p^{\prime}}$. Since $\mathcal{Q}$ is an orthogonal projection on $\boldsymbol{S}_{2}, \mathcal{Q}$ is a self-adjoint operator on $\boldsymbol{S}_{2}$. We denote the adjoint operator $\mathcal{Q}^{*}$ on $\boldsymbol{S}_{p^{\prime}}$ by the same symbol $\mathcal{Q}$.

Thus we can consider the projection $\mathcal{Q}$ on any class $\boldsymbol{S}_{p}$ with $1<p<\infty$. It is easy to show that if $T$ is an integral operator with kernel function $k$ of class $\boldsymbol{S}_{p}$, $2<p<\infty$, then $\mathcal{Q} T=Q_{\varphi}$, where $\varphi$ is defined by (11.1) and $Q_{\varphi} \in \boldsymbol{S}_{p}$. We are going to prove that for any $T \in \boldsymbol{S}_{p}, 2<p<\infty$, the operator $\mathcal{Q} T$ has the form $Q_{\varphi}$ for a function $\varphi \in X_{p}$.

Theorem 11.2. Let $T$ be an operator of class $\boldsymbol{S}_{p}, 2<p<\infty$. Then there exists a function $\varphi \in X_{p}$ such that $\mathcal{Q} T=Q_{\varphi}$.

Proof. Let $\mathfrak{X}_{p}$ be the space of operators of the form $Q_{\varphi}$ with $\varphi \in X_{p}$. Clearly, $\mathfrak{X}_{p}$ is a Banach space with norm

$$
\left\|Q_{\varphi}\right\|_{\mathfrak{X}_{p}}=\|\varphi\|_{X_{p}}
$$

It follows from Theorems 11.1 and 3.3 that for $T \in \boldsymbol{S}_{2}$

$$
\|\mathcal{Q} T\|_{\mathfrak{X}_{p}} \leq \text { const } \underset{50}{\mathcal{Q} T} \|_{\boldsymbol{S}_{p}} \leq \text { const }\|T\|_{\boldsymbol{S}_{p}}
$$


Since $\boldsymbol{S}_{2}$ dense in $\boldsymbol{S}_{p}$, it follows that $\mathcal{Q} T \in \mathfrak{X}_{p}$ for any $T \in \boldsymbol{S}_{p}$.

We consider now the behavior of $\mathcal{Q}$ on $\boldsymbol{S}_{1}$. It follows from Theorem 11.1 that if $T \in \boldsymbol{S}_{1}$, then $\mathcal{Q} T \in \boldsymbol{S}_{p}$ for any $p>1$. The next result shows that $\mathcal{Q} T$ does not have to be in $\boldsymbol{S}_{1}$ but it has to be in $\boldsymbol{S}_{1, \infty}$.

Theorem 11.3. (i) There exists an operator $T$ in $\boldsymbol{S}_{1}$ such that $\mathcal{Q} T \notin \boldsymbol{S}_{1}$.

(ii) $\mathcal{Q}$ has weak type $(1,1)$, i.e., $\mathcal{Q}$ maps $\boldsymbol{S}_{1}$ into $\boldsymbol{S}_{1, \infty}$, i.e.,

$$
s_{n}(\mathcal{Q} T) \leq \operatorname{const}(1+n)^{-1}\|T\|_{\boldsymbol{S}_{1}}, \quad T \in \boldsymbol{S}_{1} .
$$

Lemma 11.4.

$$
\mathcal{Q} \boldsymbol{S}_{1}=\mathfrak{X}_{1} \stackrel{\text { def }}{=}\left\{Q_{\varphi}: \varphi \in X_{1}\right\}
$$

Let us first deduce Theorem 11.3 from Lemma 11.4.

Proof of Theorem 11.3. (i) is an immediate consequence of Lemma 11.4 and the Example following Theorem 6.5. (ii) also follows immediately from Lemma 11.4 and Theorem 10.1.

Proof of Lemma 11.4. Let us first show that $\mathcal{Q} \boldsymbol{S}_{1} \subset \mathfrak{X}_{1}$. Let $T \in \boldsymbol{S}_{1}$ and $\mathcal{Q} T=Q_{\varphi}$. We have to prove that $\varphi \in X_{1}$. Consider the space $Z_{\infty}^{0}$ that consists of functions $f$ on $\mathbb{R}_{+}$such that

$$
\lim _{n \rightarrow \pm \infty}\left(2^{-n} \int_{2^{n}}^{2^{n+1}}|f(x)|^{2} d x\right)=0 .
$$

It is not difficult to see that $\left(Z_{\infty}^{0}\right)^{*}=X_{1}$ with respect to the pairing (11.2). As in the proof of Theorem 11.1 we define the function $\psi$ by $\psi(x)=\frac{f(x)}{2 x}, x>0$. It follows from (11.3) that

$$
|(\varphi, f)| \leq \text { const }\|T\|_{S_{1}}\left\|Q_{\psi}\right\| \leq \text { const }\|T\|_{S_{1}}\|\psi\|_{X_{\infty}^{0}} \leq \text { const }\|T\|_{S_{1}}\|f\|_{Z_{\infty}^{0}},
$$

and so $\varphi$ determines a continuous linear functional on $Z_{\infty}^{0}$. Hence, $\varphi \in X_{1}$.

To prove that $\mathcal{Q} \boldsymbol{S}_{1}=\mathfrak{X}_{1}$, we consider the operator $A: \boldsymbol{S}_{1} \rightarrow X_{1}$ defined by $A T=\varphi$, where $\varphi$ is the function on $\mathbb{R}_{+}$such that $\mathcal{Q} T=Q_{\varphi}$. We have to show that $A$ maps $\boldsymbol{S}_{1}$ onto $X_{1}$. Consider the conjugate operator $A^{*}: X_{1}^{*} \rightarrow \mathcal{B}\left(L^{2}\left(\mathbb{R}_{+}\right)\right.$).

It is easy to see that with respect to the pairing (11.2) the space $X_{1}^{*}$ can be identified with the space $Z_{\infty}$ that consists of functions $f$ on $\mathbb{R}_{+}$such that

$$
\sup _{n \in \mathbb{Z}} 2^{-n / 2}\left(\int_{2^{n}}^{2^{n+1}}|f(x)|^{2} d x\right)^{1 / 2}<\infty .
$$

Consider the operator $J: X_{1}^{*} \rightarrow X_{\infty}$ defined by $(J f)(x)=\frac{f(x)}{2 x}, x>0$. It is easy to see that $J$ maps isomorphically $X_{1}^{*}$ onto $X_{\infty}$.

It can easily be verified that $A^{*} f=Q_{J f}$. It follows from Theorem 3.1 that $\left\|A^{*} f\right\| \geq \mathrm{const}\|f\|_{X_{1}^{*}}$. It follows that $A$ maps $\boldsymbol{S}_{1}$ onto $X_{1}$. 
Remark. In [Pel3] metric properties of the averaging projection $\mathcal{P}$ onto the space of Hankel matrices were studied. In particular, it was shown in [Pel3] that $\mathcal{P} \boldsymbol{S}_{1} \subset \boldsymbol{S}_{1,2}$. However, it turns out that the averaging projection $\mathcal{Q}$ onto the operators $Q_{\varphi}$ has different properties. Theorem 11.3 shows that $\mathcal{Q}_{1} \subset \boldsymbol{S}_{1, \infty}$ but it follows from Lemma 11.4 and the remark preceding Theorem 6.6 that $\mathcal{Q} \boldsymbol{S}_{1} \not \subset \boldsymbol{S}_{1, q}$ for any $q<\infty$.

Recall that the Matsaev ideal $\boldsymbol{S}_{\omega}$ consists of the operators $T$ on Hilbert space such that

$$
\|T\|_{S_{\omega}} \stackrel{\text { def }}{=} \sum_{n \geq 0} \frac{s_{n}(T)}{1+n}<\infty .
$$

It is easy to see that $\boldsymbol{S}_{p} \subset \boldsymbol{S}_{\omega}$ for any $p<\infty$.

Consider now the operator ideal $\boldsymbol{S}_{\Omega}$ defined by (10.1). It is easy to see that $\boldsymbol{S}_{1, \infty} \subset \boldsymbol{S}_{\Omega}$. It is well known (see [GK1]) that $\boldsymbol{S}_{\omega}^{*}=\boldsymbol{S}_{\Omega}$ with respect to the pairing

$$
\{T, R\}=\operatorname{trace} T R, \quad T \in \boldsymbol{S}_{\omega}, \quad R \in \boldsymbol{S}_{\Omega} .
$$

Theorem 11.5. The averaging projection $\mathcal{Q}$ defined on $\boldsymbol{S}_{2}$ extends to a bounded linear operator from $\boldsymbol{S}_{\omega}$ to the space of compact operators. If $T \in \boldsymbol{S}_{\omega}$, then $\mathcal{Q} T=Q_{\varphi}$ for a function $\varphi$ in $X_{\infty}^{0}$.

Proof. Let us prove that $\mathcal{Q}$ extends to a bounded operator from $\boldsymbol{S}_{\omega}$ to the space of compact operators. The proof of the second part of the theorem is the same as the proof of Theorem 11.2. Since the finite rank operators are dense in $\boldsymbol{S}_{\omega}$, it is sufficient to show that $\mathcal{Q}$ extends to a bounded operator from $\boldsymbol{S}_{\omega}$ to $\mathcal{B}\left(L^{2}\left(\mathbb{R}_{+}\right)\right)$.

Let $T \in \boldsymbol{S}_{2}$ and $R \in \boldsymbol{S}_{1}$. By Theorem 11.3, $\mathcal{Q} R \in \boldsymbol{S}_{1, \infty} \subset \boldsymbol{S}_{\Omega}$. We have

$$
\{\mathcal{Q} T, R\}=\{T, \mathcal{Q} R\},
$$

and so

$$
\begin{aligned}
|\{\mathcal{Q} T, R\}| & \leq \text { const }\|T\|_{\boldsymbol{S}_{\omega}}\|\mathcal{Q} R\|_{\boldsymbol{S}_{\Omega}} \\
& \leq \text { const }\|T\|_{\boldsymbol{S}_{\omega}}\|\mathcal{Q} R\|_{\boldsymbol{S}_{1, \infty}} \leq \mathrm{const}\|T\|_{\boldsymbol{S}_{\omega}}\|R\|_{\boldsymbol{S}_{1}}
\end{aligned}
$$

by Theorem 11.3. Since $\boldsymbol{S}_{1}^{*}=\mathcal{B}\left(L^{2}\left(\mathbb{R}_{+}\right)\right)$with respect to the pairing (11.4), it follows that $\|\mathcal{Q} T\| \leq$ const $\|T\|_{S_{\omega}}$, and so $\mathcal{Q}$ extends to a bounded linear operator from $\boldsymbol{S}_{\omega}$ to $\mathcal{B}\left(L^{2}\left(\mathbb{R}_{+}\right)\right.$).

\section{Finite rank}

We say that $\varphi$ is a step function if there exist finitely many numbers $0=x_{0}<x_{1}<\cdots<x_{N}<\infty$ such that $\varphi$ is a.e. constant on each interval 
$\left(x_{i-1}, x_{i}\right)$, and zero on $\left(x_{N}, \infty\right)$. The number of steps of $\varphi$ then is the smallest possible $N$ in this definition.

There is a natural correspondence between operators $Q_{\varphi}$ where the symbol $\varphi$ is a step function, with given $x_{1}<\cdots<x_{N}$, and matrices of the form $\left\{a_{\max \{i, j\}}\right\}$. We need a simple result for such matrices, but will not pursue their study further.

Lemma 12.1. If $a_{1}, \ldots, a_{n}$ are complex numbers, then the matrix $\left\{a_{\max \{i, j\}}\right\}_{1 \leq i, j \leq n}$ has determinant $a_{n} \prod_{i=1}^{n-1}\left(a_{i}-a_{i+1}\right)$.

Proof. Denote this determinant by $D\left(a_{1}, \ldots, a_{n}\right)$. Subtracting the last row from all others, we see that $D\left(a_{1}, \ldots, a_{n}\right)=a_{n} D\left(a_{1}-a_{n}, \ldots, a_{n-1}-a_{n}\right)$, and the result follows by induction.

Theorem 12.2. $Q_{\varphi}$ has finite rank if and only if $\varphi$ is a step function. In this case, the rank of $Q_{\varphi}$ equals the number of steps of $\varphi$.

Proof. If $\varphi$ is a step function with $N$ steps, we have $\varphi=\sum_{i=1}^{N} a_{i} \chi_{\left(0, x_{i}\right)}$ for some $a_{i}$ and $x_{i}>0$, and thus $Q_{\varphi}$ is a linear combination of $N$ rank one operators.

Conversely, suppose that $\operatorname{rank}\left(Q_{\varphi}\right)=M<\infty$. Suppose that $n>M$ and that $z_{1}<\cdots<z_{n}$ are Lebesgue points of $\varphi$. If $h>0$ and $f_{z, h}=h^{-1} \chi_{(z, z+h)}$, then the matrix $\left(\left\langle Q_{\varphi} f_{z_{i}, h}, f_{z_{j}, h}\right\rangle\right)_{i j}$ has rank at most $M<n$ and thus its determinant vanishes. As $h \rightarrow 0$, as shown in the proof of Theorem 4.1, $\left\langle Q_{\varphi} f_{z_{i}, h}, f_{z_{j}, h}\right\rangle \rightarrow$ $\varphi\left(\max \left\{z_{i}, z_{j}\right\}\right)=\varphi\left(z_{\max \{i, j\}}\right)$, and thus the determinant of $\left(\varphi\left(z_{\max \{i, j\}}\right)\right)_{i j}$ vanishes too. By Lemma 12.1, this implies that either $\varphi\left(z_{i}\right)=\varphi\left(z_{i+1}\right)$ for some $i<n$ or $\varphi\left(z_{n}\right)=0$.

Consequently, if $z_{1}<\cdots<z_{n}$ are Lebesgue points of $\varphi$ such that $\varphi\left(z_{i}\right) \neq \varphi\left(z_{i+1}\right)$ for $i<n$ and $\varphi\left(z_{n}\right) \neq 0$, then $n \leq M$. Choose such a sequence $z_{1}<\cdots<z_{n}$ with $n$ maximal. If $z \in\left(z_{i}, z_{i+1}\right) \cap \operatorname{Leb}(\varphi)$ for some $i<n$, then either $\varphi(z)=\varphi\left(z_{i}\right)$ or $\varphi(z)=\varphi\left(z_{i+1}\right)$, since $n$ is maximal. Moreover, for the same reason, if $\varphi(z)=\varphi\left(z_{i}\right)$, then $\varphi\left(z^{\prime}\right)=\varphi\left(z_{i}\right)$ for every $z^{\prime} \in\left(z_{i}, z\right) \cap \operatorname{Leb}(\varphi)$, and if $\varphi(z)=\varphi\left(z_{i+1}\right)$, then $\varphi\left(z^{\prime}\right)=\varphi\left(z_{i+1}\right)$ for every $z^{\prime} \in\left(z, z_{i+1}\right) \cap \operatorname{Leb}(\varphi)$. Together with similar arguments for the intervals $\left(0, z_{1}\right)$ and $\left(z_{n}, \infty\right)$, which we leave to the reader, this easily shows that $\varphi$ is a step function with at most $n$ steps.

\section{A class of integral operators on $L^{2}(\mathbb{R})$}

In this section we associate with the operator $Q_{\varphi}$ on $L^{2}\left(\mathbb{R}_{+}\right)$an integral operator on $L^{2}(\mathbb{R})$ and we study these operators. 
For a function $\varphi \in L_{\text {loc }}^{2}\left(\mathbb{R}_{+}\right)$we define the function $\varphi^{\odot}$ on $\mathbb{R}$ by

$$
\varphi^{\searrow}(t) \stackrel{\text { def }}{=} 2 \varphi\left(e^{2 t}\right) e^{2 t}, \quad t \in \mathbb{R}
$$

this defines a one-to-one correspondence between $L_{\text {loc }}^{2}\left(\mathbb{R}_{+}\right)$and $L_{\text {loc }}^{2}(\mathbb{R})$. With a function $\psi$ on $L^{2}(\mathbb{R})$ we associate the function $\breve{\psi}$ on $\mathbb{R} \times \mathbb{R}$ defined by

$$
\breve{\psi}(s, t)=\psi(\max \{s, t\}) e^{-|s-t|}, \quad s, t \in \mathbb{R},
$$

and denote by $K_{\psi}$ the integral operator on $L^{2}(\mathbb{R})$ with kernel function $\breve{\psi}$ (if it makes sense):

$$
\left(K_{\psi} f\right)(s)=\int_{\mathbb{R}} \psi(\max \{s, t\}) e^{-|s-t|} f(t) d t .
$$

Theorem 13.1. Let $\varphi \in L_{\mathrm{loc}}^{2}\left(\mathbb{R}_{+}\right)$. Then the operators $Q_{\varphi}$ and $K_{\varphi^{\odot}}$ are unitarily equivalent.

Theorem 13.1 certainly means that the boundedness of one of the operators implies the boundedness of the other one.

Proof. Consider the unitary operator $U: L^{2}\left(\mathbb{R}_{+}\right) \rightarrow L^{2}(\mathbb{R})$ defined as follows $(U f)(t)=\sqrt{2} f\left(e^{2 t}\right) e^{t}$. It remains to observe that $K_{\varphi^{\odot}} U=U Q_{\varphi}$.

We can identify $L^{2}\left(\mathbb{R}_{+}\right)$with the subspace of $L^{2}(\mathbb{R})$ which consists of the functions vanishing on $(-\infty, 0)$. We can now extend in a natural way the operator of triangular projection $\mathcal{P}$ to act on the space of operators on $L^{2}(\mathbb{R})$ by defining it in the same way as it has been done in $\S 2$. We keep the same notation, $\mathcal{P}$, for this extension. We put $K_{\psi}^{+} \stackrel{\text { def }}{=} \mathcal{P} K_{\psi}$ and $K_{\psi}^{-} \stackrel{\text { def }}{=} K_{\psi}-\mathcal{P} K_{\psi}^{+}$.

It is easily seen from the proof of Theorem 13.1 that the operators $Q_{\varphi}^{+}$and $Q_{\varphi}^{-}$ are unitarily equivalent to the operators $K_{\varphi^{\infty}}^{+}$and $K_{\varphi^{\circ}}^{-}$respectively.

It is easy to see that, for any $p>0$,

$$
\varphi \in X_{p} \Leftrightarrow \sum_{n=-\infty}^{\infty}\left\|\varphi^{\bigcirc}\right\|_{L^{2}[n, n+1]}^{p}<\infty
$$

(and correspondingly for $X_{\infty}$ and $X_{\infty}^{0}$ ) and, using Theorem 5.1 (vii),

$$
\varphi \in Y_{p} \Leftrightarrow \sum_{n=-\infty}^{\infty}\left\|\varphi^{\bigodot}\right\|_{B V[n, n+1]}^{p}<\infty .
$$

(In (13.3) and (13.4), the intervals $[n, n+1]$ can be replaced by any partition of $\mathbb{R}$ into intervals of the same length.) We can thus translate results from the preceding sections to $K_{\psi}$, for example as follows.

Theorem 13.2. Let $\psi \in L_{\mathrm{loc}}^{2}(\mathbb{R})$. The following are equivalent:

(i) $K_{\psi}$ is bounded on $L^{2}(\mathbb{R})$; 
(ii) $K_{\psi}^{+}$is bounded on $L^{2}(\mathbb{R})$;

(iii) $\sup _{n \in \mathbb{Z}} \int_{n}^{n+1}|\psi(x)|^{2} d x<\infty$.

Proof. The theorem is a direct consequence of Theorems 3.1 and 13.1.

Similarly we find from Theorems 3.3,6.2, and 5.1, respectively, the following three theorems.

Theorem 13.3. Let $\psi \in L_{\mathrm{loc}}^{2}(\mathbb{R})$. If $1<p<\infty$, the following are equivalent:

(i) $K_{\psi} \in \boldsymbol{S}_{p}$;

(ii) $K_{\psi}^{+} \in \boldsymbol{S}_{p}$;

(iii) $\sum_{n \in \mathbb{Z}}\|\psi\|_{L^{2}[n, n+1]}^{p}<\infty$.

Theorem 13.4. If $K_{\psi} \in \boldsymbol{S}_{1}$, then $\sum_{n \in \mathbb{Z}}\|\psi\|_{L^{2}[n, n+1]}<\infty$ and thus $\psi \in L^{1}(\mathbb{R})$. Moreover, then trace $K_{\psi}=\int_{\mathbb{R}} \psi(x) d x$.

Theorem 13.5. If $1 / 2<p \leq 1$ and $\sum_{n \in \mathbb{Z}}\|\psi\|_{B V[n, n+1]}^{p}<\infty$, then $K_{\psi} \in \boldsymbol{S}_{p}$.

The following two results involving the modulus of continuity also can be obtained by changes of variables in the corresponding Theorems 10.5 and 10.9, using (10.3) and Lemma 10.3, but the details are involved and we prefer to imitate the proofs.

Theorem 13.6. If $\psi \in L^{2}(\mathbb{R})$ has support on $[0,1]$, then

$$
s_{n}\left(K_{\psi}\right) \leq C \frac{1}{n} \omega_{\psi}^{(2)}\left(\frac{1}{n}\right)+C \frac{1}{n^{2}}\|\psi\|_{L^{2}}, \quad n \geq 1 .
$$

Proof. We interpolate using Lemma 10.4 as in the proof of Theorem 10.5.

Theorem 13.7. Let $1 / 2<p \leq 1$. If $\sum_{n \in \mathbb{Z}}\|\psi\|_{L^{2}[n, n+1]}^{p}<\infty$ and

$$
\sum_{n=-\infty}^{\infty} \int_{0}^{1}\left(\omega_{\psi}^{(2)}(t ;[n, n+1])\right)^{p} t^{p-2} d t<\infty
$$

then $K_{\psi} \in \boldsymbol{S}_{p}$.

Proof. We argue as in the proofs of Theorems 10.7 and 10.9, using Theorem 13.6.

We denote by $\mathcal{F}$ the Fourier transformation on $L^{2}\left(\mathbb{R}^{n}\right)$, which is a unitary operator defined by $(2.3)$ for $f \in L^{2}\left(\mathbb{R}^{n}\right) \cap L^{1}\left(\mathbb{R}^{n}\right)$. Let $T$ be the integral operator with kernel function $k \in L^{2}\left(\mathbb{R}^{2}\right)$. Denote by $R$ the integral operator with kernel function $\mathcal{F} k$. The following lemma has a straightforward verification. 
Lemma 13.8. $\mathcal{F} T \mathcal{F}=R$.

Note that Lemma 13.8 is similar to Lemma 2.3.

Corollary 13.9. Let $p>0$. Then $\|T\|_{\boldsymbol{S}_{p}}=\|R\|_{\boldsymbol{S}_{p}}$.

Denote by $Z$ be the integral operator with kernel function $(x, y) \mapsto(\mathcal{F} k)(x,-y)$. By Lemma 2.3, $T$ is unitarily equivalent to $Z$. Indeed, the equality $\mathcal{F} T \mathcal{F}=R$ implies $\mathcal{F T F}^{-1}=Z$.

Lemma 13.10. If $\psi \in L^{1}(\mathbb{R})$, then $\breve{\psi} \in L^{1}\left(\mathbb{R}^{2}\right)$, and

$$
(\mathcal{F} \breve{\psi})(x, y)=(\mathcal{F} \psi)(x+y)\left(\frac{1}{1-2 \pi \mathrm{i} x}+\frac{1}{1-2 \pi \mathrm{i} y}\right)
$$

Proof. The inclusion $\breve{\psi} \in L^{1}\left(\mathbb{R}^{2}\right)$ is obvious. We have

$$
\begin{aligned}
(\mathcal{F} \breve{\psi})(x, y)= & \int_{\mathbb{R}^{2}} \psi(\max \{s, t\}) e^{-|s-t|} e^{-2 \pi \mathrm{i} s x-2 \pi \mathrm{i} t y} d s d t \\
= & \int_{\mathbb{R}}\left(\psi(s) e^{-s} e^{-2 \pi \mathrm{i} s x} \int_{-\infty}^{s} e^{t-2 \pi \mathrm{i} t y} d t\right) d s \\
& +\int_{\mathbb{R}}\left(\psi(t) e^{-t} e^{-2 \pi \mathrm{i} t y} \int_{-\infty}^{t} e^{s-2 \pi \mathrm{i} s x} d s\right) d t \\
= & \int_{\mathbb{R}} \frac{\psi(s) e^{-2 \pi \mathrm{i} s(x+y)} d s}{1-2 \pi \mathrm{i} y}+\int_{\mathbb{R}} \frac{\psi(t) e^{-2 \pi \mathrm{i} t(x+y)} d t}{1-2 \pi \mathrm{i} x} \\
= & \left(\frac{1}{1-2 \pi \mathrm{i} x}+\frac{1}{1-2 \pi \mathrm{i} y}\right)(\mathcal{F} \psi)(x+y) .
\end{aligned}
$$

Consider the functions

$$
\breve{\psi}_{+}(s, t) \stackrel{\text { def }}{=} \chi_{\{(s, t): s>t\}} \breve{\psi}(s, t) \text { and } \breve{\psi}_{-}(s, t) \stackrel{\text { def }}{=} \chi_{\{(s, t): s<t\}} \breve{\psi}(s, t) \text {. }
$$

It can easily be seen from the proof of Lemma 13.10 that

$$
\left(\mathcal{F} \breve{\psi}_{+}\right)(x, y)=\frac{(\mathcal{F} \psi)(x+y)}{1-2 \pi \mathrm{i} y} \quad \text { and } \quad\left(\mathcal{F} \breve{\psi}_{-}\right)(x, y)=\frac{(\mathcal{F} \psi)(x+y)}{1-2 \pi \mathrm{i} x}
$$

It is easy to verify that if $\psi$ is a tempered distribution on $\mathbb{R}$ (see $\S 2$ ), we can define tempered distributions $\breve{\psi}, \breve{\psi}_{+}$, and $\breve{\psi}_{-}$by (13.2) and (13.6); the formal definitions are by duality and analogous to (2.4)-(2.6). It is easy to check that formulas (13.5) and (13.7) also hold for tempered distributions $\psi$.

As a corollary to Theorem 13.2, we have the following lemma. 
Lemma 13.11. Let $\psi \in L_{\text {loc }}^{2}(\mathbb{R})$. Suppose that the operator $K_{\psi}$ is bounded on $L^{2}(\mathbb{R})$. Then $\psi$ determines a tempered distribution.

Theorem 13.12. Let $0<p<\infty$. Suppose that $\varphi \in L_{\text {loc }}^{2}\left(\mathbb{R}_{+}\right)$and $\varphi^{\ominus}$ is defined by (13.1). The following are equivalent:

(i) $Q_{\varphi} \in \boldsymbol{S}_{p}$;

(ii) $K_{\varphi^{\circ}} \in \boldsymbol{S}_{p}$;

(iii) the integral operator on $L^{2}(\mathbb{R})$ with kernel

$$
(x, y) \mapsto\left(\mathcal{F} \varphi^{\oslash}\right)(x+y)\left(\frac{1}{1-2 \pi \mathrm{i} x}+\frac{1}{1-2 \pi \mathrm{i} y}\right)
$$

belongs to $\boldsymbol{S}_{p}$.

Proof. The theorem is a consequence of Theorem 13.1, Lemma 13.8 and Lemma 13.10.

Note that if for $p>2$ we have a tempered distribution in (iii) rather than a function, by the integral operator we mean the operator determined by this tempered distribution ( see $\S 2$ ).

In the same way one can prove the following result.

Theorem 13.13. Let $0<p<\infty$ and let $\varphi \in L_{\text {loc }}^{2}\left(\mathbb{R}_{+}\right)$. The following are equivalent:

(i) $Q_{\varphi}^{+} \in \boldsymbol{S}_{p}$;

(ii) $Q_{\varphi}^{-} \in \boldsymbol{S}_{p}$;

(iii) $K_{\varphi^{0}}^{+} \in \boldsymbol{S}_{p}$;

(iv) $K_{\varphi^{\rho}}^{-} \in \boldsymbol{S}_{p}$

(v) the integral operator on $L^{2}(\mathbb{R})$ with the kernel

$$
(x, y) \mapsto \frac{\left(\mathcal{F} \varphi^{\odot}\right)(x+y)}{1-2 \pi \mathrm{i} x}
$$

belongs to $\boldsymbol{S}_{p}$.

It is straightforward to show that if $p>0$ and the integral operator on $L^{2}(\mathbb{R})$ with kernel function $\frac{h(x+y)}{x+\alpha}$ belongs to $\boldsymbol{S}_{p}$ for some $\alpha \in \mathbb{C} \backslash \mathbb{R}$, then it belongs to $\boldsymbol{S}_{p}$ for any $\alpha \in \mathbb{C} \backslash \mathbb{R}$. Let us show that such an integral operator can belong to $\boldsymbol{S}_{p}$ for $p \leq 1$ only if it is zero.

Consider the operator $E: \mathcal{D}\left(\mathbb{R}^{2}\right) \rightarrow \mathcal{D}\left(\mathbb{R}_{+} \times \mathbb{R}_{+}\right)$defined by the following formula $(E f)(s, t) \stackrel{\text { def }}{=} \frac{1}{2}(s t)^{-1 / 2} f\left(\frac{1}{2} \log s, \frac{1}{2} \log t\right)$. Clearly, $E$ is an isomorphism. Consequently, the conjugate operator $E^{\prime}$ is an isomorphism from $\mathcal{D}^{\prime}\left(\mathbb{R}_{+} \times \mathbb{R}_{+}\right)$ 
onto $\mathcal{D}^{\prime}\left(\mathbb{R}^{2}\right)$. Clearly, $\left(E^{\prime} \Phi\right)(x, y)=2 \Phi\left(e^{2 x}, e^{2 y}\right) e^{x} e^{y}$ if $\Phi \in L_{\text {loc }}^{1}\left(\mathbb{R}_{+} \times \mathbb{R}_{+}\right)$. Put $2 \Phi\left(e^{2 x}, e^{2 y}\right) e^{x} e^{y} \stackrel{\text { def }}{=}\left(E^{\prime} \Phi\right)(x, y)$ for $\Phi \in \mathcal{D}^{\prime}\left(\mathbb{R}_{+} \times \mathbb{R}_{+}\right)$.

Theorem 13.14. Let $\Phi \in \mathcal{D}^{\prime}\left(\mathbb{R}_{+} \times \mathbb{R}_{+}\right)$. Then $\Phi$ determines a bounded operator on $L^{2}\left(\mathbb{R}_{+}\right)$if and only if the distribution $2 \Phi\left(e^{2 x}, e^{2 y}\right) e^{x} e^{y}$ determines a bounded operator on $L^{2}(\mathbb{R})$. Moreover, these two operators are unitarily equivalent operators.

Proof. It suffices to note that

$$
\left\langle 2 \Phi\left(e^{2 x}, e^{2 y}\right) e^{x} e^{y}, \sqrt{2} e^{y} f\left(e^{2 y}\right) \overline{\sqrt{2} e^{x} g\left(e^{2 x}\right)}\right\rangle=\langle\Phi(s, t), f(t) \overline{g(s)}\rangle
$$

for any $f, g \in \mathcal{D}\left(\mathbb{R}_{+} \times \mathbb{R}_{+}\right)$, and the map $h \mapsto \sqrt{2} e^{x} h\left(e^{2 x}\right)$ is a unitary operator from $L^{2}\left(\mathbb{R}_{+}\right)$onto $L^{2}(\mathbb{R})$.

Theorem 13.15. Let $h \in \mathcal{D}^{\prime}(\mathbb{R})$. Suppose that the distribution $\frac{h(x+y)}{1-2 \pi \mathrm{i} x}$ determines an operator on $L^{2}(\mathbb{R})$ of class $\boldsymbol{S}_{1}$. Then $h=0$.

Proof. By Lemma 2.2, $\frac{h(x+y)}{1-2 \pi \mathrm{i} x} \in \mathcal{S}^{\prime}\left(\mathbb{R}^{2}\right)$. Consequently, $h(x+y) \in \mathcal{S}^{\prime}\left(\mathbb{R}^{2}\right)$, whence $h \in \mathcal{S}^{\prime}(\mathbb{R})$. Thus, there exists a distribution $\varphi \in \mathcal{D}^{\prime}\left(\mathbb{R}_{+}\right)$such that

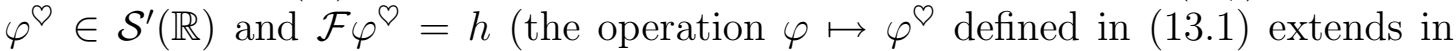
an obvious way to distributions $\varphi$ ). Lemma 2.3 and formula (13.7) imply that $Q_{\varphi}^{-}$ and thus also $Q_{\varphi}^{+}$belongs to $\boldsymbol{S}_{1}$. By Theorem 2.4, $\varphi \in L_{\mathrm{loc}}^{2}\left(\mathbb{R}_{+}\right)$. Thus $\varphi=0$ by Theorem 6.6.

We are going to prove now that for $p>1 / 2$ if the integral operator with kernel function $h(x+y)\left(\frac{1}{x+\alpha}+\frac{1}{y+\beta}\right)$ belongs to $\boldsymbol{S}_{p}$ for some $\alpha, \beta \in \mathbb{C} \backslash \mathbb{R}$ with $\alpha+\beta \notin \mathbb{R}$, then it belongs to $\boldsymbol{S}_{p}$ for any $\alpha, \beta \in \mathbb{C} \backslash \mathbb{R}$. We will also show that this is not true for $p \leq 1 / 2$.

Lemma 13.16. The function

$$
(x, y) \mapsto \frac{1}{x+y+\mathrm{i}} \chi_{[0,1]}(x), \quad x, y \in \mathbb{R},
$$

is a Schur multiplier of $\boldsymbol{S}_{p}$ for any $p>0$.

Proof. First we prove that the function

$$
(x, y) \mapsto \frac{1}{x+y+\mathrm{i}} \chi_{[0,1]}(x) \chi_{\mathbb{R} \backslash[-2,2]}(y), \quad x, y \in \mathbb{R},
$$

is a Schur multiplier of $\boldsymbol{S}_{p}$. We have

$$
\frac{1}{x+y+\mathrm{i}} \chi_{[0,1]}(x) \chi_{\mathbb{R} \backslash[-2,2]}(y)=\sum_{n \geq 0}(-1)^{n} \frac{(x+\mathrm{i})^{n}}{y^{n+1}} \chi_{[0,1]}(x) \chi_{\mathbb{R} \backslash[-2,2]}(y) .
$$

Clearly, the $p$-multiplier norm of the $n$-th summand is bounded by $2^{-n / 2}$. Consequently, the function (13.8) is a Schur multiplier of $\boldsymbol{S}_{p}$. It remains to prove that 
the function

$$
(x, y) \mapsto \frac{1}{x+y+\mathrm{i}} \chi_{[0,1]}(x) \chi_{[-2,2]}(y), \quad x, y \in \mathbb{R},
$$

is a Schur multiplier of $\boldsymbol{S}_{p}$. For any $(\xi, \eta) \in[0,1] \times[-2,2]$ we can expand the function $\frac{1}{x+y+\mathrm{i}}$ in a Taylor series in a neighborhood of $(\xi, \eta)$. It follows easily that for a sufficiently small $\varepsilon>0$ the function

$$
(x, y) \mapsto \frac{1}{x+y+\mathrm{i}} \chi_{[0,1] \cap[\xi-\varepsilon, \xi+\varepsilon]}(x) \chi_{[-2,2] \cap[\eta-\varepsilon, \eta+\varepsilon]}(y), \quad x, y \in \mathbb{R},
$$

is a Schur multiplier of $\boldsymbol{S}_{p}$. It remains to choose a finite subcover of $[0,1] \times[-2,2]$ that consists of rectangles of the form $[\xi-\varepsilon, \xi+\varepsilon] \times[\eta-\varepsilon, \eta+\varepsilon]$.

Remark. In the same way we can prove that the function

$$
(x, y) \mapsto \frac{1}{x+y+\alpha} \chi_{[\xi, \eta]}(x), \quad x, y \in \mathbb{R},
$$

is a Schur multiplier of $\boldsymbol{S}_{p}$ for any $p>0$ for any $\alpha \in \mathbb{C} \backslash \mathbb{R}$ and for any $\xi, \eta \in \mathbb{R}$.

Corollary 13.17. Let $\alpha, \beta, \gamma \in \mathbb{C}$ and $\gamma \notin \mathbb{R}$. Then the function

$$
(x, y) \mapsto \frac{(x+\alpha)(y+\beta)}{x+y+\gamma} \chi_{[0,1]}(x), \quad x, y \in \mathbb{R},
$$

is a Schur multiplier of $\boldsymbol{S}_{p}$ for any $p>0$.

Proof. We have

$$
\frac{(x+\alpha)(y+\beta)}{x+y+\gamma}=(x+\alpha)\left(1+\frac{\beta-\gamma-x}{x+y+\gamma}\right) .
$$

It remains to note that

$$
\chi_{[0,1]}(x), \quad(x+\alpha) \chi_{[0,1]}(x), \quad(\beta-\gamma-x) \chi_{[0,1]}(x), \quad \text { and } \quad \frac{1}{x+y+\gamma} \chi_{[0,1]}(x)
$$

are Schur multipliers of $\boldsymbol{S}_{p}$.

Corollary 13.18. Let $p>0$ and let $\alpha, \beta \in \mathbb{C} \backslash \mathbb{R}$ such that $\alpha+\beta \notin \mathbb{R}$. Suppose that the integral operator on $L^{2}(\mathbb{R})$ with kernel function

$$
(x, y) \mapsto h(x+y)\left(\frac{1}{x+\alpha}+\frac{1}{y+\beta}\right), \quad x, y \in \mathbb{R},
$$

belongs to $\boldsymbol{S}_{p}$. Then the integral operator with kernel function

$$
(x, y) \mapsto h(x+y) \chi_{[0,1]}(x), \quad x, y \in \mathbb{R},
$$

belongs to $\boldsymbol{S}_{p}$. 
Theorem 13.19. Let $p>1 / 2$ and let $\alpha_{0}, \beta_{0} \in \mathbb{C} \backslash \mathbb{R}$ such that $\alpha_{0}+\beta_{0} \notin \mathbb{R}$. Suppose that the integral operator on $L^{2}(\mathbb{R})$ with kernel function

$$
(x, y) \mapsto h(x+y)\left(\frac{1}{x+\alpha_{0}}+\frac{1}{y+\beta_{0}}\right), \quad x, y \in \mathbb{R},
$$

belongs to $\boldsymbol{S}_{p}$. Then the integral operator with kernel function

$$
(x, y) \mapsto h(x+y)\left(\frac{1}{x+\alpha}+\frac{1}{y+\beta}\right), \quad x, y \in \mathbb{R},
$$

also belongs to $\boldsymbol{S}_{p}$ for any $\alpha, \beta \in \mathbb{C} \backslash \mathbb{R}$.

Proof. By Corollary 13.18, the integral operator with kernel $h(x+y) \chi_{[0,1]}(x)$ belongs to $\boldsymbol{S}_{p}$. Obviously, for any $n \in \mathbb{Z}$,

$$
\left\|h(x+y) \chi_{[0,1]}(x)\right\|_{\boldsymbol{S}_{p}}=\left\|h(x+y) \chi_{[n, n+1]}(x)\right\|_{\boldsymbol{S}_{p}}
$$

(as usual we write $\|k\|_{\boldsymbol{S}_{p}}$ for the $\boldsymbol{S}_{p}$ norm (or quasi-norm) of the integral operator with kernel $k$ ). Consequently,

$$
\left\|h(x+y)\left(\frac{1}{x+\alpha}-\frac{1}{x+\alpha_{0}}\right) \chi_{[n, n+1]}(x)\right\|_{S_{p}} \leq \operatorname{const}(1+|n|)^{-2} .
$$

It is now clear that the integral operator with kernel function

$$
(x, y) \mapsto h(x+y)\left(\frac{1}{x+\alpha}-\frac{1}{x+\alpha_{0}}\right), \quad x, y \in \mathbb{R},
$$

belongs to $\boldsymbol{S}_{p}$ for $p>1 / 2$. Similarly, we prove that the integral operator with kernel function

$$
(x, y) \mapsto h(x+y)\left(\frac{1}{y+\beta}-\frac{1}{y+\beta_{0}}\right), \quad x, y \in \mathbb{R},
$$

belongs to $\boldsymbol{S}_{p}$ for $p>1 / 2$.

Theorem 13.20. Let $\varphi \in L_{\text {loc }}^{2}\left(\mathbb{R}_{+}\right), a \in \mathbb{R} \backslash\{0\}$, and $p>1 / 2$. Put

$$
\varphi_{[a]}(t) \stackrel{\text { def }}{=} \varphi\left(t^{a}\right) t^{a-1} \text {. }
$$

Then $Q_{\varphi} \in \boldsymbol{S}_{p}$ if and only if $Q_{\varphi_{[a]}} \in \boldsymbol{S}_{p}$.

Proof. Recall that $\varphi^{\searrow}(t)=2 \varphi\left(e^{2 t}\right) e^{2 t}$ and $\varphi_{[a]}^{\bigcirc}(t)=2 \varphi_{[a]}\left(e^{2 t}\right) e^{2 t}=2 \varphi\left(e^{2 a t}\right) e^{2 a t}$. Consequently, $\varphi_{[a]}^{\curvearrowright}(t)=\varphi^{\ominus}(a t)$. By Theorem 13.12, $Q_{\varphi_{[a]}} \in \boldsymbol{S}_{p}$ if and only if the integral operator on $L^{2}(\mathbb{R})$ with kernel

$$
(x, y) \mapsto\left(\mathcal{F} \varphi^{\curlyvee}\right)\left(\frac{x+y}{a}\right)\left(\frac{1}{1-2 \pi \mathrm{i} x}+\frac{1}{1-2 \pi \mathrm{i} y}\right), \quad x, y \in \mathbb{R},
$$


belongs to $\boldsymbol{S}_{p}$, and thus if and only if the integral operator on $L^{2}(\mathbb{R})$ with kernel

$$
(x, y) \mapsto\left(\mathcal{F} \varphi^{\bigotimes}\right)(x+y)\left(\frac{1}{1-2 \pi \mathrm{i} a x}+\frac{1}{1-2 \pi \mathrm{i} a y}\right), \quad x, y \in \mathbb{R},
$$

belongs to $\boldsymbol{S}_{p}$. By Theorem 13.19, this holds if and only if the integral operator on $L^{2}(\mathbb{R})$ with kernel

$$
(x, y) \mapsto\left(\mathcal{F} \varphi^{\bigotimes}\right)(x+y)\left(\frac{1}{1-2 \pi \mathrm{i} x}+\frac{1}{1-2 \pi \mathrm{i} y}\right), \quad x, y \in \mathbb{R},
$$

belongs to $\boldsymbol{S}_{p}$. It remains to apply Theorem 13.12 once more.

Corollary 13.21. Let $\psi \in L_{\mathrm{loc}}^{2}(\mathbb{R}), a \in \mathbb{R} \backslash\{0\}$, and $p>1 / 2$. Define $\psi_{a}(t) \stackrel{\text { def }}{=} \psi(a t)$. Then $K_{\psi} \in \boldsymbol{S}_{p}$ if and only if $K_{\psi_{a}} \in \boldsymbol{S}_{p}$.

Remark. Theorem 13.20 and its corollary do not generalize to the case $p \leq 1 / 2$. Indeed, if $\varphi$ is the characteristic function of an interval, then $Q_{\varphi} \in \boldsymbol{S}_{p}$ for any $p>0$ but if $a \neq 1$, then $Q_{\varphi_{[a]}} \notin \boldsymbol{S}_{p}$ by Corollary 8.12. It follows from the proof above that Theorem 13.19 too does not extend to $p \leq 1 / 2$.

Remark. Note that if $\varphi \in X_{p}$, then $\varphi_{[a]}(t) \in X_{p}$ for any $a \in \mathbb{R} \backslash\{0\}$ and any $p>0$. Moreover, if $\varphi \in Y_{p}$, then $\varphi_{[a]}(t) \in Y_{p}$ for any $a \in \mathbb{R} \backslash\{0\}$ and any $p>0$. Indeed, let $A>1$. It is easy to see that (1.3) is equivalent to the condition

$$
\sum_{n \in \mathbb{Z}} A^{n p / 2}\left(\int_{A^{n}}^{A^{n+1}}|\varphi(x)|^{2} d x\right)^{p / 2}<\infty,
$$

while the condition in Theorem 5.3 (vii) is equivalent to

$$
\sum_{n \in \mathbb{Z}}\|x \varphi(x)\|_{B V\left[A^{n}, A^{n+1}\right]}^{p}<\infty,
$$

which easily implies the above assertions.

Theorem 13.22. Let $\alpha, \beta \in \mathbb{C} \backslash \mathbb{R}$ and let $p>0$. Then the integral operator on $L^{2}(\mathbb{R})$ with kernel function

$$
(x, y) \mapsto h(x+y)\left(\frac{1}{x+\alpha}+\frac{1}{y+\beta}\right), \quad x, y \in \mathbb{R},
$$

belongs to $\boldsymbol{S}_{p}$ if and only if convolution with the function $h(x)(x+\alpha+\beta)$ is an operator from $L^{2}\left(\mathbb{R},\left(1+x^{2}\right) d x\right)$ to $L^{2}\left(\mathbb{R},\left(1+x^{2}\right)^{-1} d x\right)$ of class $\boldsymbol{S}_{p}$.

Proof. Clearly, the integral operator on $L^{2}(\mathbb{R})$ with kernel function

$$
(x, y) \mapsto h(x+y)\left(\frac{1}{x+\alpha}+\frac{1}{y+\beta}\right), \quad x, y \in \mathbb{R},
$$


belongs to $\boldsymbol{S}_{p}$ if and only if so does the integral operator with kernel function

$$
(x, y) \mapsto h(x-y) \frac{x-y+\alpha+\beta}{(x+\alpha)(y-\beta)}, \quad x, y \in \mathbb{R} .
$$

To complete the proof, it remains to observe that multiplication by $(x-\beta)^{-1}$ is an isomorphism from $L^{2}(\mathbb{R})$ onto $L^{2}\left(\mathbb{R},\left(1+x^{2}\right) d x\right)$ and multiplication by $(x+\alpha)^{-1}$ is an isomorphism from $L^{2}\left(\mathbb{R},\left(1+x^{2}\right)^{-1} d x\right)$ onto $L^{2}(\mathbb{R})$.

Corollary 13.23. Let $\alpha, \beta, \gamma \in \mathbb{C} \backslash \mathbb{R}$ such that $\alpha+\beta \notin \mathbb{R}$ and let $p>1 / 2$. Then the integral operator with kernel function

$$
(x, y) \mapsto h(x+y)\left(\frac{1}{x+\alpha}+\frac{1}{y+\beta}\right), \quad x, y \in \mathbb{R},
$$

belongs to $\boldsymbol{S}_{p}$ if and only if convolution with the function $h(x)(x+\gamma)$ is an operator from $L^{2}\left(\mathbb{R},\left(1+x^{2}\right) d x\right)$ to $L^{2}\left(\mathbb{R},\left(1+x^{2}\right)^{-1} d x\right)$ of class $\boldsymbol{S}_{p}$.

Proof. It suffices to apply Theorem 13.19.

Remark. In the same way we can prove that the following statements are equivalent for any $\alpha \in \mathbb{C} \backslash \mathbb{R}$ and for any $p>0$ :

(i) the integral operator on $L^{2}(\mathbb{R})$ with kernel function

$$
(x, y) \mapsto k(x+y)(x+\alpha)^{-1}, \quad x, y \in \mathbb{R},
$$

belongs to $\boldsymbol{S}_{p}$;

(ii) the integral operator on $L^{2}(\mathbb{R})$ with kernel function

$$
(x, y) \mapsto k(x+y)(y+\alpha)^{-1}, \quad x, y \in \mathbb{R},
$$

belongs to $\boldsymbol{S}_{p}$;

(iii) convolution with $k$ is an operator from $L^{2}(\mathbb{R})$ to $L^{2}\left(\mathbb{R},\left(1+x^{2}\right)^{-1} d x\right)$ of class $\boldsymbol{S}_{p}$

(iv) convolution with $k$ is an operator from $L^{2}\left(\mathbb{R},\left(1+x^{2}\right) d x\right)$ to $L^{2}(\mathbb{R})$ of class $\boldsymbol{S}_{p}$

Let us repeat that Theorem 13.15 implies that the integral operator with kernel function $k(x+y)(x+\alpha)^{-1}$ can be a nonzero operator in $\boldsymbol{S}_{p}$ only if $p>1$.

\section{Matrix representation}


Let $\varphi$ be a function in $L_{\text {loc }}^{2}(\mathbb{R})$ such that $\varphi(x+1)=\varphi(x), x \in \mathbb{R}$. Consider the operators $Q_{\varphi}^{[0,1]}$ and $Q_{\varphi}^{[0,2]}$ on $L^{2}[0,1]$ and $L^{2}[0,2]$ respectively. Obviously,

$$
\left\|Q_{\varphi}^{[0,1]}\right\|_{\boldsymbol{S}_{p}} \leq\left\|Q_{\varphi}^{[0,2]}\right\|_{\boldsymbol{S}_{p}}, \quad 0<p<\infty .
$$

Obviously,

$$
\left\|Q_{\varphi}^{[0,2]}\right\| \geq\left\|\mathcal{P}_{[1,2] \times[0,1]} Q_{\varphi}^{[0,2]}\right\|=\|\varphi\|_{L^{2}[0,1]} .
$$

It is also easy to see that

$$
\left\|Q_{\varphi}^{[0,2]}\right\|_{\boldsymbol{S}_{p}} \leq C(p)\left(\left\|Q_{\varphi}^{[0,1]}\right\|_{\boldsymbol{S}_{p}}+\|\varphi\|_{L^{2}[0,1]}\right), \quad 0<p<\infty
$$

where $C(p)$ is a constant that may depend only on $p$.

Theorem 14.1. Let $0<p<\infty$. Suppose that $\varphi$ is a function in $L_{\mathrm{loc}}^{2}(\mathbb{R})$ such that $\varphi(x)=\varphi(x+1)$ and $\psi(x) \stackrel{\text { def }}{=} \varphi(1-x)$. Then

$$
C_{1}(p)\left(\left\|Q_{\varphi}^{[0,1]}\right\|_{\boldsymbol{S}_{p}}+\left\|Q_{\psi}^{[0,1]}\right\|_{\boldsymbol{S}_{p}}\right) \leq\left\|Q_{\varphi}^{[0,2]}\right\|_{\boldsymbol{S}_{p}} \leq C_{2}(p)\left(\left\|Q_{\varphi}^{[0,1]}\right\|_{\boldsymbol{S}_{p}}+\left\|Q_{\psi}^{[0,1]}\right\|_{\boldsymbol{S}_{p}}\right) \text {. }
$$

Proof. To prove the left inequality, consider the integral operator $K$ on $L^{2}[0,1]$ with kernel function

$$
(x, y) \mapsto \varphi(\min \{x, y\}) .
$$

Clearly, the operators $K$ and $Q_{\psi}^{[0,1]}$ are unitarily equivalent, and so $\|K\|_{\boldsymbol{S}_{p}}=\left\|Q_{\psi}^{[0,1]}\right\|_{\boldsymbol{S}_{p}}$ for any $p>0$. Note that

$$
\varphi(\min \{x, y\})+\varphi(\max \{x, y\})=\varphi(x)+\varphi(y) .
$$

Hence, $K+Q_{\varphi}^{[0,1]}$ is the integral operator with kernel function $(x, y) \mapsto \varphi(x)+\varphi(y)$. Thus $\left\|Q_{\varphi}^{[0,1]}+K\right\|_{\boldsymbol{S}_{p}} \leq C(p)\|\varphi\|_{L^{2}}$. Now the left inequality is obvious. To prove the right inequality, we have to show that

$$
\|\varphi\|_{L^{2}[0,1]} \leq C(p)\left(\left\|Q_{\varphi}^{[0,1]}\right\|_{\boldsymbol{S}_{p}}+\left\|Q_{\psi}^{[0,1]}\right\|_{\boldsymbol{S}_{p}}\right) .
$$

Clearly,

$$
\left(K+Q_{\varphi}^{[0,1]}\right) 1=\varphi+\int_{0}^{1} \varphi(t) d t
$$

It follows that

$$
\left\|\left(K+Q_{\varphi}^{[0,1]}\right) 1\right\|_{L^{2}[0,1]}^{2}=\|\varphi\|_{L^{2}[0,1]}^{2}+3\left|\int_{0}^{1} \varphi(t) d t\right|^{2} \geq\|\varphi\|_{L^{2}[0,1]}^{2} .
$$

Thus,

$$
\left\|K+Q_{\varphi}^{[0,1]}\right\|_{S_{p}} \geq\left\|K+Q_{\varphi}^{[0,1]}\right\| \geq\|\varphi\|_{L^{2}[0,1]}
$$


and so

$$
\begin{aligned}
\|\varphi\|_{L^{2}[0,1]} & \leq\left\|\left(K+Q_{\varphi}^{[0,1]}\right)\right\|_{\boldsymbol{S}_{p}} \\
& \leq C(p)\left(\|K\|_{\boldsymbol{S}_{p}}+\left\|Q_{\varphi}^{[0,1]}\right\|_{\boldsymbol{S}_{p}}\right) \\
& =C(p)\left(\left\|Q_{\varphi}^{[0,1]}\right\|_{\boldsymbol{S}_{p}}+\left\|Q_{\psi}^{[0,1]}\right\|_{\boldsymbol{S}_{p}}\right) .
\end{aligned}
$$

Corollary 14.2. Under the hypotheses of Theorem 14.1

$$
\left\|Q_{\psi}^{[0,2]}\right\|_{\boldsymbol{S}_{p}} \leq C(p)\left\|Q_{\varphi}^{[0,2]}\right\|_{\boldsymbol{S}_{p}} .
$$

Theorem 14.3. Let $p>0$ and $a \in \mathbb{R}$. If $\varphi$ is a function satisfying the hypotheses of Theorem 14.1 and $\psi(x) \stackrel{\text { def }}{=} \varphi(x-a)$, then

$$
\left\|Q_{\psi}^{[0,2]}\right\|_{\boldsymbol{S}_{p}} \leq C(p)\left\|Q_{\varphi}^{[0,2]}\right\|_{\boldsymbol{S}_{p}} .
$$

Proof. Clearly, it is sufficient to consider the case $a \in(0,1)$. Then $Q_{\psi}^{[0,1]}$ is by a translation unitarily equivalent to $Q_{\varphi}^{[1-a, 2-a]}$, and thus

$$
\left\|Q_{\psi}^{[0,1]}\right\|_{\boldsymbol{S}_{p}}=\left\|Q_{\varphi}^{[1-a, 2-a]}\right\|_{\boldsymbol{S}_{p}} \leq\left\|Q_{\varphi}^{[0,2]}\right\|_{\boldsymbol{S}_{p}} .
$$

The result follows by (14.3) and (14.2).

Let $\phi$ be a function on the unit circle $\mathbb{T}$. Put $\mathbb{T}_{+} \stackrel{\text { def }}{=}\{\zeta \in \mathbb{T}: \operatorname{Im} \zeta \geq 0\}$, $\mathbb{T}_{-} \stackrel{\text { def }}{=}\{\zeta \in \mathbb{T}: \operatorname{Im} \zeta<0\}$ and

$$
k_{\phi}(\zeta, \tau) \stackrel{\text { def }}{=} \begin{cases}\phi\left(\zeta^{2}\right), & \zeta \bar{\tau} \in \mathbb{T}_{+}, \quad(\zeta, \tau) \in \mathbb{T}^{2} \\ \phi\left(\tau^{2}\right), & \zeta \bar{\tau} \in \mathbb{T}_{-},\end{cases}
$$

It is easy to see that the functions $\phi$ and $k_{\phi}$ are equimeasurable. In particular, $\|\phi\|_{L^{2}(\mathbb{T})}=\left\|k_{\phi}\right\|_{L^{2}\left(\mathbb{T}^{2}\right)}$. Note also that if $\phi$ is continuous on $\mathbb{T}$, then $k_{\phi}$ is continuous on $\mathbb{T}^{2}$. Let $\phi \in L^{2}(\mathbb{T})$. Denote by $K_{\phi}$ the integral operator on $L^{2}(\mathbb{T})$ with kernel function $k_{\phi}$.

Theorem 14.4. Let $p>0$. Suppose that $\phi \in L^{2}(\mathbb{T})$ and $\varphi(t) \stackrel{\text { def }}{=} \phi\left(e^{2 \pi \mathrm{i} t}\right), t \in \mathbb{R}$. Then

$$
C_{1}(p)\left\|Q_{\varphi}^{[0,2]}\right\|_{\boldsymbol{S}_{p}} \leq\left\|K_{\phi}\right\|_{\boldsymbol{S}_{p}} \leq C_{2}(p)\left\|Q_{\varphi}^{[0,2]}\right\|_{\boldsymbol{S}_{p}},
$$

where $C_{1}(p)$ and $C_{2}(p)$ may depend only on $p$.

Proof. Consider the integral operator $K$ on $L^{2}[0,1]$ with kernel function $k \in L^{2}\left([0,1]^{2}\right)$ defined by

$$
k(x, y)=\left\{\begin{array}{cc}
\varphi(2 \max \{x, y\}), & |x-y| \leq 1 / 2 \\
\varphi(2 \min \{x, y\}), & |x-y|>1 / 2 \\
64
\end{array}\right.
$$


It is easy to see that $K$ is unitarily equivalent to $K_{\phi}$. For $\alpha, \beta=0,1$ we consider the integral operator $K^{(\alpha, \beta)}$ with kernel function

$$
(x, y) \mapsto k(x, y) \chi_{[\alpha / 2,(1+\alpha) / 2]}(x) \chi_{[\beta / 2,(1+\beta) / 2]}(y) .
$$

Using the substitution $(x, y) \mapsto(2 x, 2 y)$, we find that $2\left\|K^{(0,0)}\right\|_{\boldsymbol{S}_{p}}=\left\|Q_{\varphi}^{[0,1]}\right\|_{\boldsymbol{S}_{p}}$. In a similar way we can obtain $2\left\|K^{(1,1)}\right\|_{\boldsymbol{S}_{p}}=\left\|Q_{\varphi}^{[0,1]}\right\|_{\boldsymbol{S}_{p}}$. Let $\psi(t) \stackrel{\text { def }}{=} \varphi(1-t)$. It is also easy to see that $2\left\|K^{(0,1)}\right\|_{\boldsymbol{S}_{p}}=2\left\|K^{(1,0)}\right\|_{\boldsymbol{S}_{p}}=\left\|Q_{\psi}^{[0,1]}\right\|_{\boldsymbol{S}_{p}}$. Hence,

$$
\begin{aligned}
\frac{1}{4}\left(\left\|Q_{\varphi}^{[0,1]}\right\|_{\boldsymbol{S}_{p}}+\left\|Q_{\psi}^{[0,1]}\right\|_{\boldsymbol{S}_{p}}\right) & =\frac{1}{4} \sum_{\alpha=0}^{1} \sum_{\beta=0}^{1}\left\|K^{(\alpha, \beta)}\right\|_{\boldsymbol{S}_{p}} \\
& \leq\left\|K_{\phi}\right\|_{\boldsymbol{S}_{p}} \leq C(p) \sum_{\alpha=0}^{1} \sum_{\beta=0}^{1}\left\|K^{(\alpha, \beta)}\right\|_{\boldsymbol{S}_{p}} \\
& =C(p)\left(\left\|Q_{\varphi}^{[0,1]}\right\|_{\boldsymbol{S}_{p}}+\left\|Q_{\psi}^{[0,1]}\right\|_{\boldsymbol{S}_{p}}\right) .
\end{aligned}
$$

It remains to apply Theorem 14.1.

We denote by $\hat{f}(n)$ denote the $n$th Fourier coefficient of a function $f$ in $L^{1}(\mathbb{T})$. For convenience we put

$$
\hat{f}(n+1 / 2) \stackrel{\text { def }}{=} 0, \quad n \in \mathbb{Z} .
$$

For a function $k$ in $L^{1}\left(\mathbb{T}^{2}\right)$ we denote by $\{\hat{k}(m, n)\}_{(m, n) \in \mathbb{Z}^{2}}$ the sequence of its Fourier coefficients.

Let $\phi$ be a function on $\mathbb{T}$. Put

$$
k_{\phi}^{+}(\zeta, \tau) \stackrel{\text { def }}{=} \begin{cases}\phi\left(\zeta^{2}\right), & \zeta \bar{\tau} \in \mathbb{T}_{+}, \\ 0, & \zeta \bar{\tau} \in \mathbb{T}_{-}\end{cases}
$$

and

$$
k_{\phi}^{-}(\zeta, \tau) \stackrel{\text { def }}{=} \begin{cases}0, & \zeta \bar{\tau} \in \mathbb{T}_{+}, \\ \phi\left(\tau^{2}\right), & \zeta \bar{\tau} \in \mathbb{T}_{-} .\end{cases}
$$

Clearly, $k_{\phi}^{+}(\zeta, \tau)=k_{\phi}^{-}(\tau, \zeta)$ and $k_{\phi}(\zeta, \tau)=k_{\phi}^{+}(\zeta, \tau)+k_{\phi}^{-}(\zeta, \tau)$, where $k_{\phi}$ is defined by (14.4).

Lemma 14.5. Let $\phi \in L^{1}(\mathbb{T})$. Then for $(m, n) \in \mathbb{Z}^{2}$

$$
\hat{k}_{\phi}^{+}(m, n)= \begin{cases}\frac{1}{2} \hat{\phi}\left(\frac{m}{2}\right), & n=0, \\ \frac{\mathrm{i}}{\pi n} \hat{\phi}\left(\frac{m+n}{2}\right), & m, n \text { are odd } \\ 0, & \text { otherwise }\end{cases}
$$


Proof. Let us first observe that for any $\zeta \in \mathbb{T}$ and $n \in \mathbb{Z}$ we have

$$
\int_{\left\{\tau \in \mathbb{T}: \zeta \bar{\tau} \in \mathbb{T}_{+}\right\}} \tau^{-n} d \boldsymbol{m}(\tau)= \begin{cases}\frac{1}{2}, & n=0, \\ \frac{\mathrm{i}}{\pi n} \zeta^{-n}, & n \text { is odd, } \\ 0, & \text { otherwise. }\end{cases}
$$

It follows that

$$
\begin{aligned}
\int_{\mathbb{T}} k_{\phi}^{+}(\zeta, \tau) \zeta^{-m} \tau^{-n} d \boldsymbol{m}(\zeta) & =\int_{\left\{\tau \in \mathbb{T}: \zeta \bar{\tau} \in \mathbb{T}_{+}\right\}} \phi\left(\zeta^{2}\right) \zeta^{-m} \tau^{-n} d \boldsymbol{m}(\tau) \\
& = \begin{cases}\frac{1}{2} \phi\left(\zeta^{2}\right) \zeta^{-m}, & n=0, \\
\frac{i}{\pi n} \phi\left(\zeta^{2}\right) \zeta^{-m-n}, & n \text { is odd }, \\
0, & \text { otherwise. }\end{cases}
\end{aligned}
$$

It remains to integrate the last identity in $\zeta$.

Corollary 14.6. Let $\phi \in L^{1}(\mathbb{T})$. Then

$$
\hat{k}_{\phi}^{-}(m, n)= \begin{cases}\frac{1}{2} \hat{\phi}\left(\frac{n}{2}\right), & m=0, \\ \frac{\mathrm{i}}{\pi m} \hat{\phi}\left(\frac{m+n}{2}\right), & m, n \text { are odd }, \\ 0, & \text { otherwise. }\end{cases}
$$

Proof. It suffices to observe that $k_{\phi}^{-}(\zeta, \tau)=k_{\phi}^{+}(\tau, \zeta)$.

Corollary 14.7. Let $\phi \in L^{1}(\mathbb{T})$. Then

$$
\hat{k}_{\phi}(m, n)= \begin{cases}\frac{1}{2} \hat{\phi}\left(\frac{m}{2}\right), & n=0, \\ \frac{1}{2} \hat{\phi}\left(\frac{n}{2}\right), & m=0, \\ \hat{\phi}(0), & m=n=0, \\ \frac{\mathrm{i}}{\pi}\left(\frac{1}{m}+\frac{1}{n}\right) \hat{\phi}\left(\frac{m+n}{2}\right), & m, n \text { are odd }, \\ 0, & \text { otherwise. }\end{cases}
$$

Proof. It suffices to observe that $k_{\phi}(\zeta, \tau)=k_{\phi}^{+}(\zeta, \tau)+k_{\phi}^{+}(\tau, \zeta)$.

Theorem 14.8. Let $p>0$. Suppose that $\varphi(t)=\sum_{k \in \mathbb{Z}} a_{k} e^{2 \pi \mathrm{i} k t}$ and $\sum_{k \in \mathbb{Z}}\left|a_{k}\right|^{2}<\infty$. Then $Q_{\varphi}^{[0,2]} \in \boldsymbol{S}_{p}$ if and only if the matrix

$$
\left\{a_{m+n+1}\left(\frac{1}{m+\frac{1}{2}}+\frac{1}{n+\frac{1}{2}}\right)\right\}_{m, n \in \mathbb{Z}}
$$

belongs to $\boldsymbol{S}_{p}$. 
Here we identify operators on $\ell^{2}(\mathbb{Z})$ with their matrices with respect to the standard orthonormal basis of $\ell^{2}(\mathbb{Z})$.

Proof. Consider the function $\phi$ on $\mathbb{T}$ defined by $\phi(z)=\sum_{n \in \mathbb{Z}} a_{n} z^{n}$. By Theorem 14.4, $Q_{\varphi}^{[0,2]} \in \boldsymbol{S}_{p}$ if and only if $K_{\phi} \in \boldsymbol{S}_{p}$. It easy to see that the operator $K_{\phi}$ belongs to $\boldsymbol{S}_{p}$ if and only if the matrix $\left\{\hat{k}_{\phi}(m, n)\right\}_{m, n \in \mathbb{Z}}$ belongs to $\boldsymbol{S}_{p}$. Corollary 14.7 implies that $\hat{k}_{\phi}(m, n) \neq 0$ only if $m n$ is odd or $m n=0$. Hence, it is easy to check that $\left\{\hat{k}_{\phi}(m, n)\right\}_{m, n \in \mathbb{Z}} \in \boldsymbol{S}_{p}$ if and only if $\left\{\hat{k}_{\phi}(2 m+1,2 n+1)\right\}_{m, n \in \mathbb{Z}} \in \boldsymbol{S}_{p}$. It remains to note that

$$
\hat{k}_{\phi}(2 m+1,2 n+1)=\frac{\mathrm{i}}{\pi}\left(\frac{1}{2 m+1}+\frac{1}{2 n+1}\right) a_{m+n+1}
$$

by Corollary 14.7.

Clearly, the same reasoning shows that $Q_{\varphi}^{[0,2]}$ is bounded on $L^{2}[0,1]$ if and only if the matrix (14.5) is bounded. The following result shows that the boundedness of (14.5) is equivalent to its membership of $\boldsymbol{S}_{p}, p>1$.

Theorem 14.9. Let $\left\{a_{k}\right\}_{k \in \mathbb{Z}}$ be a two-sided sequence of complex numbers and let

$$
A \stackrel{\text { def }}{=}\left\{a_{m+n+1}\left(\frac{1}{m+\frac{1}{2}}+\frac{1}{n+\frac{1}{2}}\right)\right\}_{m, n \in \mathbb{Z}} .
$$

Suppose that $p>1$. The following are equivalent:

(i) $A$ is a bounded operator on $\ell^{2}(\mathbb{Z})$;

(ii) $A \in \boldsymbol{S}_{p}$;

(iii) $\left\{a_{k}\right\}_{k \in \mathbb{Z}} \in \ell^{2}(\mathbb{Z})$.

Proof. Suppose that $A$ is bounded. Then the sequence

$$
\left\{a_{n+1}\left(2+\frac{1}{n+\frac{1}{2}}\right)\right\}_{n \in \mathbb{Z}}
$$

belongs to $\ell^{2}(\mathbb{Z})$ which implies (iii). Clearly, (iii) is equivalent to the fact that $\varphi \in L^{2}[0,1]$. By Theorem 3.3, $Q_{\varphi}^{[0,2]} \in \boldsymbol{S}_{p}$, and so by Theorem 14.8, $A \in \boldsymbol{S}_{p}$. The implication (ii) $\Rightarrow$ (i) is trivial.

Related results, that matrices of a roughly similar sort are bounded if and only if they are in certain $\boldsymbol{S}_{p}$ can be found in [W1].

Remark. Note that the following identities hold:

$$
\begin{gathered}
\frac{\mathrm{i}}{\pi} \sum_{m, n \in \mathbb{Z}} a_{m+n+1}\left(\frac{1}{m+\frac{1}{2}}+\frac{1}{n+\frac{1}{2}}\right) \zeta^{m} \tau^{n}=\frac{\phi(\zeta)-\phi(\tau)}{\sqrt{\zeta \tau}}, \\
\frac{\mathrm{i}}{\pi} \sum_{m, n \in \mathbb{Z}} \frac{a_{m+n+1}}{n+\frac{1}{2}} \zeta^{m} \tau^{n}=\frac{\phi(\zeta)}{\sqrt{\zeta \tau}}
\end{gathered}
$$




$$
\frac{\mathrm{i}}{\pi} \sum_{m, n \in \mathbb{Z}} \frac{a_{m+n+1}}{m+\frac{1}{2}} \zeta^{m} \tau^{n}=-\frac{\phi(\tau)}{\sqrt{\zeta \tau}}
$$

where $\sqrt{\zeta \tau}$ is chosen so that $\bar{\tau} \sqrt{\zeta \tau} \in \mathbb{T}_{+}$(the series converge in $L^{2}\left(\mathbb{T}^{2}\right)$ ).

Indeed, it suffices to note that by Corollary 14.7,

$$
\frac{\mathrm{i}}{\pi} \sum_{m, n \in \mathbb{Z}} a_{m+n+1}\left(\frac{1}{2 m+1}+\frac{1}{2 n+1}\right) \zeta^{2 m+1} \tau^{2 n+1}=\frac{1}{2}\left(k_{\phi}(\zeta, \tau)-k_{\phi}(\zeta,-\tau)\right),
$$

by Lemma 14.5,

$$
\frac{\mathrm{i}}{\pi} \sum_{m, n \in \mathbb{Z}} \frac{a_{m+n+1}}{2 n+1} \zeta^{2 m+1} \tau^{2 n+1}=\frac{1}{2}\left(k_{\phi}^{+}(\zeta, \tau)-k_{\phi}^{+}(\zeta,-\tau)\right),
$$

and by Corollary 14.6,

$$
\frac{\mathrm{i}}{\pi} \sum_{m, n \in \mathbb{Z}} \frac{a_{m+n+1}}{2 m+1} \zeta^{2 m+1} \tau^{2 n+1}=\frac{1}{2}\left(k_{\phi}^{-}(\zeta, \tau)-k_{\phi}^{-}(\zeta,-\tau)\right) .
$$

Remark. Note that if $p>1 / 2$, then

$$
A \stackrel{\text { def }}{=}\left\{a_{m+n+1}\left(\frac{1}{m+\frac{1}{2}}+\frac{1}{n+\frac{1}{2}}\right)\right\}_{m, n \in \mathbb{Z}} \in \boldsymbol{S}_{p}
$$

if and only if

$$
B \stackrel{\text { def }}{=}\left\{a_{m+n}\left(\frac{1}{m+\frac{1}{2}}+\frac{1}{n+\frac{1}{2}}\right)\right\}_{m, n \in \mathbb{Z}} \in \boldsymbol{S}_{p}
$$

Indeed, put $\psi(t) \stackrel{\text { def }}{=} e^{2 \pi i t} \varphi(t)$. By Theorem 14.8, it suffices to prove that $Q_{\varphi}^{[0,2]} \in \boldsymbol{S}_{p}$ implies $Q_{\psi}^{[0,2]} \in \boldsymbol{S}_{p}$. This follows from Theorem 7.3.

Note however that for $p \leq 1 / 2$ this is not true. Indeed, if $a_{0}=1$ and $a_{n}=0$ for $n \neq 0$ (in other words, $\varphi(t)=1, t \in \mathbb{R}$ ), then it is easy to see that $A$ is the zero matrix, and so it belongs to $\boldsymbol{S}_{p}$ for any $p>0$. On the other hand, the matrix $B$ has nonzero entries $-\left(n^{2}-1 / 4\right)^{-1}$ for $m=-n$, and so it belongs to $\boldsymbol{S}_{p}$ only for $p>1 / 2$. The situation is similar in the case where the restriction $\varphi \mid[0,1)$ is the characteristic function of an interval in which case $A \in \boldsymbol{S}_{p}$ for any $p>0$ but $B \in \boldsymbol{S}_{p}$ only for $p>1 / 2$, see Theorem 8.11 and Corollary 9.11. 
Suppose now that $p>1 / 2$ and consider the following submatrices of the matrix $B$ defined by (14.6):

$$
\begin{aligned}
& B_{1}=\left\{a_{m+n}\left(\frac{1}{m+\frac{1}{2}}+\frac{1}{n+\frac{1}{2}}\right)\right\}_{m, n \geq 0}, \\
& B_{2}=\left\{a_{m+n}\left(\frac{1}{m+\frac{1}{2}}+\frac{1}{n+\frac{1}{2}}\right)\right\}_{m \geq 0, n<0}, \\
& B_{3}=\left\{a_{m+n}\left(\frac{1}{m+\frac{1}{2}}+\frac{1}{n+\frac{1}{2}}\right)\right\}_{m<0, n \geq 0},
\end{aligned}
$$

and

$$
B_{4}=\left\{a_{m+n}\left(\frac{1}{m+\frac{1}{2}}+\frac{1}{n+\frac{1}{2}}\right)\right\}_{m, n<0} .
$$

Clearly, $B \in \boldsymbol{S}_{p}$ if and only if all matrices $B_{j}, 1 \leq j \leq 4$, belong to $\boldsymbol{S}_{p}$.

It is direct that $B_{1} \in \boldsymbol{S}_{p}$ if and only if the matrix

$$
\left\{a_{j+k} \frac{j+k+1}{(j+1)(k+1)}\right\}_{j, k \geq 0}
$$

belongs to $\boldsymbol{S}_{p}$. Matrices of the form

$$
\left\{a_{j+k}(1+j)^{\alpha}(1+k)^{\beta}\right\}_{j, k \geq 0}
$$

are called weighted Hankel matrices. It was proved in [Pel2] that if $\alpha>-1 / 2$, $\beta>-1 / 2$, and $0<p \leq 1$, the matrix (14.8) belongs to $\boldsymbol{S}_{p}$ if and only if the function $\sum_{n>0} a_{n} z^{n}$ belongs to the Besov class $B_{p p}^{1 / p+\alpha+\beta}$ of functions on the unit circle $\mathbb{T}$. More recent results on Schatten class properties of weighted Hankel matrices are in [RW] and [W2]. However, in the case of interest, the weighted Hankel matrix (14.7) for $\alpha=\beta=-1$, no characterization of such matrices of class $\boldsymbol{S}_{p}$ is known. In the next section we obtain some necessary conditions for the matrix (14.7) to belong to $\boldsymbol{S}_{1}$.

It is also easy to see that $B_{4} \in \boldsymbol{S}_{p}$ if and only if the weighted Hankel matrix

$$
\left\{a_{-(j+k+2)} \frac{j+k+1}{(j+1)(k+1)}\right\}_{j, k \geq 0}
$$

belongs to $\boldsymbol{S}_{p}$.

It can also be easily shown that $B_{2} \in \boldsymbol{S}_{p}$ if and only if $B_{3} \in \boldsymbol{S}_{p}$ and this is equivalent to the fact that the weighted Toeplitz matrix

$$
\left\{a_{j-k-1} \frac{j-k}{(1+j)(1+k)}\right\}_{j, k \geq 0}
$$


belongs to $\boldsymbol{S}_{p}$.

Summarizing the above, we can state the following result.

Theorem 14.10. Let $1 / 2<p \leq 1$ and let $\varphi$ be a function in $L^{2}[0,1]$ of the form $\varphi(t)=\sum_{n \in \mathbb{Z}} a_{n} e^{2 \pi \mathrm{in} t}, t \in[0,1]$. Then $Q_{\varphi}^{[0,1]} \in \boldsymbol{S}_{p}$ if and only if the matrices (14.7), (14.9), and (14.10) belong to $\boldsymbol{S}_{p}$.

In the next section we use the results above to obtain necessary conditions for the nuclearity of operators $Q_{\varphi}$.

Let us consider now the family of functions $\left\{F_{\lambda}\right\}_{\lambda \in \mathbb{C}}$ on $[0,1)^{2}$ defined for a function $\varphi \in L^{1}[0,1]$ by

$$
F_{\lambda}(t, s) \stackrel{\text { def }}{=} \varphi(\max \{s, t\}) e^{-2 \pi \lambda \mathrm{i}|s-t|}+\varphi(\min \{s, t\}) e^{2 \pi \lambda \mathrm{i}|s-t|-2 \pi \lambda \mathrm{i}} .
$$

Clearly, $\quad F_{\lambda} \in L^{1}\left([0,1)^{2}\right)$. We identify $[0,1)^{2}$ with $\mathbb{T}^{2}$ via the map $(s, t) \mapsto\left(e^{2 \pi \mathrm{i} s}, e^{2 \pi \mathrm{i} t}\right)$ and we can consider the Fourier coefficients of functions on $[0,1)^{2}$.

Theorem 14.11. Suppose that $\lambda \notin \mathbb{Z}$. Then

$$
\widehat{F}_{\lambda}(m, n)=\frac{1-e^{-2 \pi \mathrm{i} \lambda}}{2 \pi \mathrm{i}}\left(\frac{1}{\lambda-m}+\frac{1}{\lambda-n}\right) \hat{\varphi}(m+n) .
$$

Proof. We have

$$
\widehat{F}_{\lambda}(m, n) \stackrel{\text { def }}{=} \iint_{[0,1) \times[0,1)} F_{\lambda}(s, t) e^{-2 \pi \mathrm{i} m s-2 \pi \mathrm{i} n t} d s d t=\iint_{t \geq s}+\iint_{t \leq s} .
$$

Let us compute the first integral:

$$
\begin{aligned}
\iint_{t \geq s}= & \int_{0}^{1}\left(\int_{0}^{t} \varphi(t) e^{2 \pi \mathrm{i} \lambda(s-t)} e^{-2 \pi \mathrm{i} m t-2 \pi \mathrm{i} n s} d s\right) d t \\
& +\int_{0}^{1}\left(\int_{s}^{1} \varphi(s) e^{2 \pi \mathrm{i} \lambda(t-s)-2 \pi \mathrm{i} \lambda} e^{-2 \pi \mathrm{i} m t-2 \pi \mathrm{i} n s} d t\right) d s \\
= & \int_{0}^{1} \varphi(t) e^{-2 \pi \mathrm{i} \lambda t-2 \pi \mathrm{i} m t}\left(\int_{0}^{t} e^{2 \pi \mathrm{i} s(\lambda-n)} d s\right) d t \\
& +\int_{0}^{1} \varphi(s) e^{-2 \pi \mathrm{i} \lambda s-2 \pi \mathrm{i} \lambda-2 \pi \mathrm{i} n s}\left(\int_{s}^{1} e^{2 \pi \mathrm{i} t(\lambda-m)} d t\right) d s
\end{aligned}
$$




$$
\begin{aligned}
& =\int_{0}^{1} \varphi(t) e^{-2 \pi \mathrm{i} \lambda t-2 \pi \mathrm{i} m t} \frac{e^{2 \pi \mathrm{i} t(\lambda-n)}-1}{2 \pi \mathrm{i}(\lambda-n)} d t \\
& \quad+\int_{0}^{1} \varphi(s) e^{-2 \pi \mathrm{i} \lambda s-2 \pi \mathrm{i} \lambda-2 \pi \mathrm{i} n s} \frac{e^{2 \pi \mathrm{i} \lambda}-e^{2 \pi \mathrm{i} s(\lambda-m)}}{2 \pi \mathrm{i}(\lambda-m)} d s \\
& =\frac{\hat{\varphi}(m+n)}{2 \pi \mathrm{i}(\lambda-n)}-\frac{1}{2 \pi \mathrm{i}(\lambda-n)} \int_{0}^{1} \varphi(t) e^{-2 \pi i \lambda t-2 \pi i m t} d t \\
& \quad+\frac{1}{2 \pi \mathrm{i}(\lambda-m)} \int_{0}^{1} \varphi(s) e^{-2 \pi \mathrm{i} \lambda s-2 \pi \mathrm{i} n s} d s-\frac{e^{-2 \pi \mathrm{i} \lambda} \hat{\varphi}(m+n)}{2 \pi \mathrm{i}(\lambda-m)} .
\end{aligned}
$$

Similarly,

$$
\begin{aligned}
\iint_{t \leq s}= & \frac{\hat{\varphi}(m+n)}{2 \pi \mathrm{i}(\lambda-m)}-\frac{1}{2 \pi \mathrm{i}(\lambda-m)} \int_{0}^{1} \varphi(s) e^{-2 \pi \mathrm{i} \lambda s-2 \pi \mathrm{i} n s} d s \\
& +\frac{1}{2 \pi \mathrm{i}(\lambda-n)} \int_{0}^{1} \varphi(t) e^{-2 \pi \mathrm{i} \lambda t-2 \pi \mathrm{i} m t} d t-\frac{e^{-2 \pi \mathrm{i} \lambda} \hat{\varphi}(m+n)}{2 \pi \mathrm{i}(\lambda-n)}
\end{aligned}
$$

which implies (14.11).

Theorem 14.12. Let $\lambda \in \mathbb{C}$. The integral operator with kernel function $F_{\lambda}$ is bounded on $L^{2}[0,1]$ if and only if $\varphi \in L^{2}([0,1))$.

Proof. Clearly, the integral operator with kernel function $F_{\lambda}$ belongs to $\boldsymbol{S}_{2}$ if $\varphi \in L^{2}([0,1))$. Suppose now that the integral operator with kernel function $F_{\lambda}$ is bounded. If $\lambda \in \mathbb{Z}$, then

$$
\begin{aligned}
F_{\lambda}(s, t) & =\left(\varphi(\max \{s, t\}) e^{-4 \pi \mathrm{i} \lambda \max \{s, t\}}+\varphi(\min \{s, t\}) e^{-4 \pi \mathrm{i} \lambda \min \{s, t\}}\right) e^{2 \pi \mathrm{i} \lambda(s+t)} \\
& =\left(\varphi(s) e^{-4 \pi \mathrm{i} \lambda s}+\varphi(t) e^{-4 \pi \mathrm{i} \lambda t}\right) e^{2 \pi \mathrm{i} \lambda(s+t)} .
\end{aligned}
$$

Consequently, the boundedness of this operator implies that $\varphi \in L^{2}[0,1]$. If $\lambda \notin \mathbb{Z}$, then the boundedness of the integral operator with kernel function $F_{\lambda}$ implies that

$$
\sum_{m \in \mathbb{Z}}\left|\widehat{F}_{\lambda}(m, 0)\right|^{2}<+\infty
$$


and by Theorem 14.11 we obtain

$$
\sum_{m \in \mathbb{Z}}|\hat{\varphi}(m)|^{2}\left|\frac{2 \lambda-m}{\lambda-m}\right|^{2}<+\infty,
$$

whence $\varphi \in L^{2}[0,1]$.

Lemma 14.13. Let $w, a \in \mathbb{C}$ and $w \neq 1$. Let $p>\frac{1}{2}$. Then the function

$$
(s, t) \mapsto\left(w-e^{a|s-t|}\right)^{-1} \chi_{\Delta}(s) \chi_{\Delta}(t), \quad s, t \in \mathbb{R},
$$

is a Schur multiplier of $\boldsymbol{S}_{p}$ if $\Delta$ is an interval of sufficiently small length.

Proof. Clearly, it suffices to consider the case $p<1$. Note that $w-e^{a|s-t|}$ is a Schur multiplier of $\boldsymbol{S}_{p}\left(L^{2}(\Delta)\right)$ by Theorem 7.3 , since

$$
w-e^{a|s-t|}=w-e^{a \max \{s, t\}} e^{-a \min \{s, t\}} .
$$

We have to prove that this multiplier is an isomorphism of $\boldsymbol{S}_{p}\left(L^{2}(\Delta)\right)$ if $\Delta$ has sufficiently small length. For $\omega \in L^{\infty}\left(\Delta^{2}\right)$ we put

$$
\|\omega\|_{\mathfrak{M}_{p}(\Delta)} \stackrel{\text { def }}{=} \sup \|\omega k\|_{\boldsymbol{S}_{p}\left(L^{2}(\Delta)\right)},
$$

where the supremum is taken over all integral operators with kernel $k \in L^{2}\left(\Delta^{2}\right)$ such that $\|k\|_{\boldsymbol{S}_{p}}=1$. Here by $\|k\|_{\boldsymbol{S}_{p}}$ we mean the $\boldsymbol{S}_{p}$ norm (quasi-norm if $p<1$ ) of the integral operator with kernel function $k$. Obviously, it suffices to prove the inequality

$$
\left\|e^{a \max \{s, t\}} e^{-a \min \{s, t\}}-1\right\|_{\mathfrak{M}_{p}(\Delta)}<|w-1|
$$

provided the length of $\Delta$ is sufficiently small. Theorem 7.7 implies that, for any $x_{0} \in \Delta$,

$$
\lim _{|\Delta| \rightarrow 0}\left\|e^{a\left(\max \{s, t\}-x_{0}\right)}-1\right\|_{\mathfrak{M}_{p}(\Delta)}=0 \quad \text { and } \quad \lim _{|\Delta| \rightarrow 0}\left\|e^{-a\left(\min \{s, t\}-x_{0}\right)}-1\right\|_{\mathfrak{M}_{p}(\Delta)}=0 .
$$

Hence, the desired inequality is obvious.

Theorem 14.14. Suppose that $\lambda \notin \mathbb{Z}$ and $p>1 / 2$. Then $Q_{\varphi}^{[0,2]} \in \boldsymbol{S}_{p}$ if and only if the integral operator with kernel function $F_{\lambda}$ belongs to $\boldsymbol{S}_{p}$.

Proof. Suppose that $Q_{\varphi}^{[0,2]} \in \boldsymbol{S}_{p}$. Then the integral operators with kernel functions $\varphi(\max \{s, t\})$ and $\varphi(\min \{s, t\})$ belong to $\boldsymbol{S}_{p}\left(L^{2}[0,1]\right)$ (see Theorem 14.1). Note that $e^{2 \pi \mathrm{i} \lambda|s-t|}=e^{2 \pi \mathrm{i} \lambda(2 \max \{s, t\}-s-t)}$. It follows now from Theorem 7.3 that the integral operator with kernel function $F_{\lambda}$ belongs to $\boldsymbol{S}_{p}$.

Suppose now that the integral operator with kernel function $F_{\lambda}$ belongs to $\boldsymbol{S}_{p}$. We have to prove that $Q_{\varphi}^{[0,2]} \in \boldsymbol{S}_{p}$. By Theorem 14.12, $\varphi \in L^{2}[0,1]$, and so it suffices to show that $Q_{\varphi}^{[0,1]} \in \boldsymbol{S}_{p}$. By Lemma 14.13, we can choose a positive number $\delta$ such that the function $\left(e^{2 \pi \mathrm{i} \lambda}-e^{4 \pi \mathrm{i} \lambda|s-t|}\right)^{-1}$ belongs to $\mathfrak{M}_{p}(\Delta)$ for any interval $\Delta$ of 
length less than $\delta$. We can represent the interval $[0,1)$ in the form $\bigcup_{j=1}^{N} \Delta_{j}$, where the $\Delta_{j}$ are pairwise disjoint intervals with lengths less than $\delta$. Clearly,

$$
F_{\lambda}(s, t)=\varphi(\max \{s, t\}) e^{-2 \pi \lambda \mathrm{i}|s-t|}+\left(\varphi(s)+\varphi(t)-\varphi(\max \{s, t\}) e^{2 \pi \lambda \mathrm{i}|s-t|-2 \pi \lambda \mathrm{i}} .\right.
$$

Let $s, t \in \Delta_{j}$. Then

$$
\begin{gathered}
\varphi(\max \{s, t\})=\frac{F_{\lambda}(s, t)-(\varphi(s)+\varphi(t)) e^{2 \pi \lambda \mathrm{i}|s-t|-2 \pi \lambda \mathrm{i}}}{e^{-2 \pi \lambda \mathrm{i}|s-t|}-e^{2 \pi \lambda \mathrm{i}|s-t|-2 \pi \lambda \mathrm{i}}}= \\
\frac{\left(F_{\lambda}(s, t)-(\varphi(s)+\varphi(t)) e^{2 \pi \lambda \mathrm{i}(\max \{s, t\}-\min \{s, t\}-1}\right)}{e^{2 \pi \lambda \mathrm{i}}-e^{4 \pi \lambda \mathrm{i}|s-t|}} e^{2 \pi \lambda \mathrm{i}(1+\max \{s, t\}-\min \{s, t\})} .
\end{gathered}
$$

Theorem 7.3 and Lemma 14.13 imply that the integral operator with kernel function

$$
(s, t) \mapsto \varphi(\max \{t, s\}) \chi_{\Delta_{j}}(t) \chi_{\Delta_{j}}(s), \quad s, \tau \in \mathbb{R},
$$

belongs to $\boldsymbol{S}_{p}$. To complete the proof, it remains to observe that the kernel function

$$
(s, t) \mapsto \varphi(\max \{s, t\})-\sum_{j=1}^{N} \varphi(\max \{s, t\}) \chi_{\Delta_{j}}(s) \chi_{\Delta_{j}}(t)
$$

determines a finite rank operator.

Theorem 14.14 implies that if $p>1 / 2, \lambda_{1}, \lambda_{2} \notin \mathbb{Z}$, and $F_{\lambda_{1}} \in \boldsymbol{S}_{p}$, then $F_{\lambda_{2}} \in \boldsymbol{S}_{p}$. This can also be easily deduced from the following elementary fact: if $x \in \ell^{2}(\mathbb{Z})$ and $y \in \ell^{p}(\mathbb{Z})$ with $p \leq 2$, then $\left\{x_{m+n} y_{n}\right\}_{m, n \in \mathbb{Z}} \in \boldsymbol{S}_{p}$.

\section{Necessary conditions for $\boldsymbol{Q}_{\varphi} \in \boldsymbol{S}_{1}$}

In this section we obtain various necessary conditions for $Q_{\varphi} \in \boldsymbol{S}_{1}$.

Theorem 15.1. Let $\left\{a_{n}\right\}_{n \geq 0}$ be a sequence in $\ell^{2}$. If the matrix

$$
\Gamma=\left\{a_{j+k}\left(\frac{1}{j+\frac{1}{2}}+\frac{1}{k+\frac{1}{2}}\right)\right\}_{j, k \geq 0}
$$

belongs to $\boldsymbol{S}_{1}$, then the function $\sum_{n \geq 0} \log (2+n) a_{n} z^{n}$ belongs to the Hardy class $H^{1}$.

We need the following well-known lemma (see e.g., [Pel1]).

Lemma 15.2. Suppose that the matrix $\left\{a_{j k}\right\}_{j, k \geq 0}$ belongs to $\boldsymbol{S}_{1}$. Then the function $\sum_{n \geq 0}\left(\sum_{j=0}^{n} a_{j n-j}\right) z^{n}$ belongs to the Hardy class $H^{1}$. 
Proof. It is sufficient to prove this when the matrix has rank one in which case the result is an immediate consequence of the fact that $H^{2} \cdot H^{2} \subset H^{1}$.

Lemma 15.3. Let $m \in \mathbb{Z}_{+}$and let

$$
\beta_{n} \stackrel{\text { def }}{=} \sum_{j=0}^{n} \frac{1}{j+\frac{1}{2}}
$$

Then there exists $d \in \mathbb{R}$ such that

$$
\left|\beta_{n}-\log (2+n)-d\right| \leq \text { const } \frac{1}{1+n} .
$$

Proof. We use the following well known fact (see, for example, [Z, Ch. I, (8.9)])

$$
\left|\sum_{j=1}^{n} \frac{1}{j}-\log n-\gamma\right| \leq \mathrm{const} \cdot n^{-1}
$$

where $\gamma$ is the Euler constant. We have

$$
\sum_{j=0}^{n} \frac{1}{j+\frac{1}{2}}=2 \sum_{j=0}^{n} \frac{1}{2 j+1}=2 \sum_{j=1}^{2 n+1} \frac{1}{j}-\sum_{j=1}^{n} \frac{1}{j}
$$

and so by (15.2),

$$
\left|\sum_{j=0}^{n} \frac{1}{j+\frac{1}{2}}-2 \log (2 n+1)+\log n-\gamma\right| \leq \operatorname{const} \cdot n^{-1}
$$

which implies the result.

Proof of Theorem 15.1. By Lemma 15.2, we have

$$
\sum_{m \geq 0} a_{m}\left(\sum_{j=0}^{m}\left(\frac{1}{j+\frac{1}{2}}+\frac{1}{\left(m-j+\frac{1}{2}\right)}\right)\right) z^{m}=2 \sum_{m \geq 0} a_{m}\left(\sum_{j=0}^{m} \frac{1}{j+\frac{1}{2}}\right) z^{m} \in H^{1} .
$$

Since $\left\{a_{n}\right\}_{n>0} \in \ell^{2}$, it is not hard to check that Lemma 15.3 implies that $\sum_{n \geq 0} \log (2+n) a_{n} z^{n} \in H^{1}$.

Theorem 15.4. Let $\varphi \in L^{2}[0, b]$ and $\varphi(x)=\sum_{n \in \mathbb{Z}} a_{n} e^{2 \pi \mathrm{in} n x / b}$. If $Q_{\varphi}^{[0, b]} \in \boldsymbol{S}_{1}$, then the functions $\sum_{n \geq 0} a_{n} \log (2+n) z^{n}$ and $\sum_{n \geq 0} a_{-n} \log (2+n) z^{n}$ in the unit disc $\mathbb{D}$ belong to the Hardy class $H^{1}$.

Proof. Without loss of generality we may assume that $b=1$. By Theorem 14.8, the matrices

$$
\Gamma=\left\{a_{j+k+1}\left(\frac{1}{j+\frac{1}{2}}+\frac{1}{k+\frac{1}{2}}\right)\right\}_{j, k \geq 0}
$$


and

$$
\Gamma=\left\{a_{-(j+k+1)}\left(\frac{1}{j+\frac{1}{2}}+\frac{1}{k+\frac{1}{2}}\right)\right\}_{j, k \geq 0}
$$

belong to $\boldsymbol{S}_{1}$. The result follows now from Theorem 15.1.

Theorem 15.5. Let $I$ be a compact interval in $(0, \infty)$ and let $\varphi$ be a function in $L_{\mathrm{loc}}^{1}\left(\mathbb{R}_{+}\right)$such that $Q_{\varphi} \in \boldsymbol{S}_{1}$. If

$$
a_{n}=\int_{I} \varphi(x) e^{-2 \pi \mathrm{i} n x /|I|} d x, \quad n \in \mathbb{Z},
$$

then the functions $\sum_{n \geq 0} a_{n} \log (2+n) z^{n}$ and $\sum_{n \geq 0} a_{-n} \log (2+n) z^{n}$ belong to the Hardy class $H^{1}$.

Proof. Since $I$ is separated away from 0 , it follows that $\varphi \mid I \in L^{2}(I)$. We can now apply a translation and reduce the result to Theorem 15.4.

Corollary 15.6. Under the hypotheses of either Theorem 15.4 or 15.5 the following holds:

(i) $\left|a_{n}\right| \leq \operatorname{const}(\log (2+|n|))^{-1}, n \in \mathbb{Z}$;

(ii) suppose that $\left\{n_{k}\right\}_{k \geq 0}$ is an Hadamard lacunary sequence of positive integers, i.e.,

then

$$
\inf _{k \geq 0} \frac{n_{k+1}}{n_{k}}>1
$$

$$
\sum_{k \geq 0}\left|a_{n_{k}}\right|^{2}\left(\log \left(1+n_{k}^{2}\right)\right)^{2}<\infty \quad \text { and } \quad \sum_{k \geq 0}\left|a_{-n_{k}}\right|^{2}\left(\log \left(1+n_{k}^{2}\right)\right)^{2}<\infty .
$$

Proof. (i) follows immediately from Theorem 15.5 and the obvious fact that the Fourier coefficients of an $H^{1}$ function are bounded. Finally, (ii) is an immediate consequence of Theorem 15.5 and Paley's inequality (see [Z, v. 2, Ch. XII, (7.8)]).

Note that if $I$ is a compact interval in $(0, \infty)$, the restrictions of function in $X_{1}$ to $I$ fill the space $L^{2}(I)$, and so the sequence of Fourier coefficients $\left\{a_{n}\right\}_{n \in \mathbb{Z}}$ can be an arbitrary sequence in $\ell^{2}$. Thus Corollary 15.6 also shows that the condition $\varphi \in X_{1}$ is not sufficient for $Q_{\varphi} \in \boldsymbol{S}_{1}$.

Now we are going to use Theorem 13.12 to obtain another necessary condition for $Q_{\varphi} \in \boldsymbol{S}_{1}$. We denote by $\mathfrak{H}^{1}$ the Stein-Weiss space of functions $f$ in $L^{1}(\mathbb{R})$ such that $\mathcal{F}^{-1}\left(\chi_{\mathbb{R}_{+}} \mathcal{F} f\right) \in L^{1}(\mathbb{R})$, where $\mathcal{F}$ is Fourier transformation.

Theorem 15.7. Let $h \in L_{\text {loc }}^{2}(\mathbb{R})$. Suppose that the integral operator on $L^{2}(\mathbb{R})$ with kernel function

$$
(x, y) \mapsto h(x, y) \stackrel{\text { def }}{=} h(x+y)\left(\frac{1}{x+\mathrm{i}}+\frac{1}{y-\mathrm{i}}\right), \quad x, y \in \mathbb{R},
$$


belongs to $\boldsymbol{S}_{1}$. Then the Fourier transform of the function $h(x) \log \left(1+x^{2}\right)$ belongs to the Stein-Weiss space $\mathfrak{H}^{1}$.

Proof. Clearly, the integral operator with kernel function $h \cdot \chi_{[0,+\infty)^{2}}$ belongs to $\boldsymbol{S}_{1}$. Put

$$
g(x) \stackrel{\text { def }}{=} \int_{\mathbb{R}} h^{\boldsymbol{\uparrow}}(t, x-t) \chi_{[0,+\infty)^{2}}(t, x-t) d t .
$$

We have

$$
g(x)= \begin{cases}h(x) \int_{0}^{x}\left(\frac{1}{t+\mathrm{i}}+\frac{1}{x-t-\mathrm{i}}\right) d t=h(x) \log \left(1+x^{2}\right), & x>0, \\ 0, & x<0 .\end{cases}
$$

It follows from Theorem 6.3 that $\mathcal{F} g \in L^{1}(\mathbb{R})$. In the same way it can be shown that the Fourier transform of the function $h(x) \log \left(1+x^{2}\right) \chi_{\mathbb{R}_{-}}(x)$ belongs to $L^{1}(\mathbb{R})$. This implies the result.

Corollary 15.8. Let $h \in L_{\text {loc }}^{2}(\mathbb{R})$ and let $a, b \in \mathbb{C} \backslash \mathbb{R}$ such that $a+b \notin \mathbb{R}$. Suppose that the integral operator on $L^{2}(\mathbb{R})$ with kernel function

$$
h_{a, b}^{\star}(x, y) \stackrel{\text { def }}{=} h(x+y)\left(\frac{1}{x+a}+\frac{1}{y+b}\right)
$$

belongs to $\boldsymbol{S}_{1}$. Then the Fourier transform of the function $h(x+c) \log \left(1+x^{2}\right)$ belongs to $\mathfrak{H}^{1}$ for any $c \in \mathbb{R}$.

Proof. Clearly, the integral operators on $L^{2}(\mathbb{R})$ with kernels functions $h(x+y+c)\left(\frac{1}{x+a+c}+\frac{1}{y+b}\right)$ belong to $\boldsymbol{S}_{1}$. Consequently, by Theorem 13.19, the integral operator on $L^{2}(\mathbb{R})$ with kernel function $h(x+y+c)\left(\frac{1}{x+\mathrm{i}}+\frac{1}{y-\mathrm{i}}\right)$ belongs to $\boldsymbol{S}_{1}$. It remains to apply Theorem 15.7.

Corollary 15.9. Suppose that $h, a$ and $b$ satisfy the hypotheses of Corollary 15.8. Then $h(x) \log |x| \rightarrow 0$ as $|x| \rightarrow \infty$.

Now Corollary 15.9 and Theorem 13.12 imply the following theorem

Theorem 15.10. Let $\varphi \in L_{\mathrm{loc}}^{2}\left(\mathbb{R}_{+}\right)$. Suppose that $Q_{\varphi} \in \boldsymbol{S}_{1}$. Then

$$
\log |x| \int_{\mathbb{R}_{+}} \varphi(t) t^{x \mathrm{i}} d t \rightarrow 0 \quad \text { as } \quad|x| \rightarrow \infty .
$$

Note that it follows from Theorem 6.2 that $\varphi \in L^{1}\left(\mathbb{R}_{+}\right)$, and so the integral in (15.3) is well defined. It is easy to see that if $\varphi$ is an arbitrary $L^{2}$ function supported on a compact subset of $(0, \infty)$, then $\varphi \in X_{1}$. However, $\varphi$ does not have to satisfy (15.3), and so Theorem 15.10 also implies that the condition $\varphi \in X_{1}$ is not sufficient for $Q_{\varphi} \in \boldsymbol{S}_{1}$. 
We conclude this section with necessary conditions on the $L^{1}$ modulus of continuity of symbols. If $f$ is a function on $\mathbb{T}$, then its $L^{1}$ modulus of continuity $\omega_{f}^{(1)}$ is defined by, in analogy with (10.2),

$$
\omega_{f}^{(1)}(t) \stackrel{\text { def }}{=} \sup _{\zeta \in \mathbb{T},|1-\zeta|<t} \int_{\mathbb{T}}|f(\zeta \tau)-f(\tau)| d \boldsymbol{m}(\tau), \quad t>0 .
$$

The following result is possibly known to experts. We were not able to find a reference, and we prove it here.

Theorem 15.11. Let $f \in L^{1}(\mathbb{T})$ and let

$$
g(z)=\sum_{n \in \mathbb{Z}} \frac{\hat{f}(n)}{\log (|n|+2)} z^{n} .
$$

Then $g \in L^{1}(\mathbb{T})$ and

$$
\lim _{t \rightarrow 0} \omega_{g}^{(1)}(t) \log \frac{1}{t}=0 .
$$

Consider the function $\mathbf{h}$ on $\mathbb{T}$ defined by

$$
\mathbf{h}(z) \stackrel{\text { def }}{=} \sum_{n \in \mathbb{Z}}(\log (|n|+2))^{-1} z^{n} .
$$

It is well-known (see, for example, $[\mathrm{Z}, \mathrm{Ch} . \mathrm{V},(1.5)]$ ) that the series converges for $z \in \mathbb{T} \backslash\{1\}, \mathbf{h} \geq 0$ and $\mathbf{h} \in L^{1}(\mathbb{T})$. We define the function $h$ on $\mathbb{R}$ by

$$
h(x) \stackrel{\text { def }}{=} \mathbf{h}\left(e^{\mathrm{i} x}\right)=(\log 2)^{-1}+2 \sum_{n \geq 1}(\log (n+2))^{-1} \cos n x .
$$

Then $h$ is continuously differentiable on $\mathbb{R} \backslash 2 \pi \mathbb{Z}$, see $[\mathrm{Z}, \mathrm{Ch}$. V, Miscellaneous theorems and examples, 7].

We use the following notation. Let $\varphi$ and $\psi$ be nonvanishing functions on an interval $(0, \alpha)$. We write

$$
\varphi \stackrel{0}{\sim} \psi, \quad \text { if } \quad \lim _{x \rightarrow 0} \frac{\varphi(x)}{\psi(x)}=1
$$

Lemma 15.12. Let $h$ be the function defined by (15.5). Then

$$
h(x) \stackrel{0}{\sim} \frac{\pi}{x(\log x)^{2}}
$$

and

$$
h^{\prime}(x) \stackrel{0}{\sim}-\frac{\pi}{x^{2}(\log x)^{2}} .
$$


Proof. (15.6) is proved in [Z, Ch. V, (2.17)]. Let us prove (15.7). Using Abel's transformation, we obtain

$$
\begin{aligned}
h(x)= & \sum_{n \geq 0}\left((\log (n+2))^{-1}-(\log (n+3))^{-1}\right) \frac{\sin \left(n+\frac{x}{2}\right)}{\sin \frac{x}{2}} \\
= & \cot \frac{x}{2}\left(\sum_{n \geq 0}\left((\log (n+2))^{-1}-(\log (n+3))^{-1}\right) \sin n x\right) \\
& +\sum_{n \geq 0}\left((\log (n+2))^{-1}-(\log (n+3))^{-1}\right) \cos n x .
\end{aligned}
$$

Consequently,

$$
\begin{aligned}
h^{\prime}(x)=- & \frac{1}{2 \sin ^{2} \frac{x}{2}}\left(\sum_{n \geq 0}\left((\log (n+2))^{-1}-(\log (n+3))^{-1}\right) \sin n x\right) \\
& +\cot \frac{x}{2}\left(\sum_{n \geq 0}\left((\log (n+2))^{-1}-(\log (n+3))^{-1}\right) n \cos n x\right) \\
& -\sum_{n \geq 0}\left((\log (n+2))^{-1}-(\log (n+3))^{-1}\right) n \sin n x \stackrel{\text { def }}{=} \Sigma_{1}+\Sigma_{2}+\Sigma_{3} .
\end{aligned}
$$

It remains to observe that

$$
\Sigma_{1} \stackrel{0}{\sim}-\frac{\pi}{x^{2}(\log x)^{2}}
$$

by $[\mathrm{Z}, \mathrm{Ch} . \mathrm{V},(2.13)]$, while

$$
\Sigma_{2} \stackrel{0}{\sim}-\frac{2 \pi}{x^{2}(\log x)^{3}}
$$

and

$$
\Sigma_{3} \stackrel{0}{\sim}-\frac{1}{x(\log x)^{2}}
$$

by $[\mathrm{Z}, \mathrm{Ch} . \mathrm{V},(2.18)]$.

Corollary 15.13. The following inequality holds

$$
\int_{\mathbb{T}}|\mathbf{h}(\zeta \tau)-\mathbf{h}(\tau)| d \boldsymbol{m}(\tau) \leq C\left(\log \frac{3}{|\zeta-1|}\right)^{-1}
$$

for any $\zeta \in \mathbb{T}$. 
Proof. It suffices to prove that

$$
\int_{-\pi}^{\pi}|h(x+t)-h(x)| d x \leq C\left(\log \frac{1}{t}\right)^{-1}
$$

for sufficiently small positive $t$. We have

$$
\begin{aligned}
\int_{-\pi}^{\pi}|h(x+t)-h(x)| d x= & \int_{|x| \leq 2 t}|h(x+t)-h(x)| d x \\
& +\int_{2 t \leq|x| \leq \pi}|h(x+t)-h(x)| d x \\
& \leq 2 \int_{|x| \leq 3 t}|h(x)| d x+\int_{2 t \leq|x| \leq \pi}|h(x+t)-h(x)| d x \\
& \stackrel{\text { def }}{=} 2 I_{1}+I_{2} .
\end{aligned}
$$

Using Lemma 15.12, we obtain

$$
I_{1} \leq C \int_{0}^{3 t} \frac{1}{x(\log x)^{2}} d x \leq C\left(\log \frac{1}{t}\right)^{-1}
$$

and

$$
I_{2} \leq C t \int_{2 t}^{\pi} \frac{d x}{x^{2}\left(\log \frac{x}{10}\right)^{2}} \leq C\left(\log \frac{1}{t}\right)^{-2},
$$

if $t>0$ is sufficiently small.

Proof of Theorem 15.11. Note that $g=f * \mathbf{h}$. Consequently, $g \in L^{1}(\mathbb{T})$. It follows easily from Corollary 15.13 that

$$
\omega_{g}^{(1)}(t) \leq \mathrm{const}\|f\|_{L^{1}}\left(\log \frac{3}{t}\right)^{-1}, \quad 0<t<2 .
$$

The result follows now from the obvious fact that (15.4) holds for trigonometric polynomials $f$.

For a function $f \in L^{1}(\mathbb{R})$, we defined the $L^{1}$ modulus of continuity $\omega_{f}^{(1)}$ in $(10.2)$ :

$$
\omega_{f}^{(1)}(t)=\sup _{|s| \leq t} \int_{\mathbb{R}}|f(x+s)-f(x)| d x, \quad t>0 .
$$


In fact this definition can be extended to functions $f$ not necessarily in $L^{1}(\mathbb{R})$. It is sufficient to assume that

$$
\int_{\mathbb{R}}|f(x+s)-f(x)| d x<\infty, \quad s \in \mathbb{R} .
$$

In a similar way we can prove the following analog of Theorem 15.11.

Theorem 15.14. Let $f \in L^{1}(\mathbb{R})$. Then there exists a function $g \in L^{1}(\mathbb{R})$ such that

$$
(\mathcal{F} f)(x)=(\mathcal{F} g(x)) \log (|x|+2), \quad x \in \mathbb{R},
$$

and

$$
\lim _{t \rightarrow 0} \omega_{g}^{(1)}(t) \log \frac{1}{t}=0
$$

Proof. Indeed, let

$$
\mathfrak{h}(x) \stackrel{\text { def }}{=} \int_{\mathbb{R}}(\log (2+|t|))^{-1} e^{-2 \pi i t x} d t=2 \int_{0}^{\infty}(\log (2+t))^{-1} \cos (2 \pi t x) d t .
$$

Then $\mathfrak{h}$ is an even positive continuously differentiable function on $\mathbb{R} \backslash\{0\}$. We can repeat the above reasoning to prove that

$$
\mathfrak{h}(x) \stackrel{0}{\sim} \frac{1}{2 x(\log x)^{2}}
$$

and

$$
\mathfrak{h}^{\prime}(x) \stackrel{0}{\sim}-\frac{1}{2 x^{2}(\log x)^{2}}
$$

Moreover, $|\mathfrak{h}(x)| \leq$ const $\cdot x^{-2}$ and $\left|\mathfrak{h}^{\prime}(x)\right| \leq$ const $\cdot x^{-2}$ everywhere. These estimates allow us to obtain the inequality

$$
\int_{\mathbb{R}}|\mathfrak{h}(x+t)-\mathfrak{h}(x)| d x \leq \operatorname{const}\left(\log \frac{1}{t}\right)^{-1}
$$

for $t \in\left(0, \frac{1}{2}\right)$ and repeat the reasoning in the proof of Theorem 15.11.

Let us introduce some more notation. Set $\mathbb{C}_{+} \stackrel{\text { def }}{=}\{z \in \mathbb{C}: \operatorname{Im} z>0\}$ and $\mathbb{C}_{-} \stackrel{\text { def }}{=}\{z \in \mathbb{C}: \operatorname{Im} z<0\}$. Let $f$ be a function in $L_{\text {loc }}^{1}(\mathbb{R})$ such that

$$
\int_{\mathbb{R}} \frac{|f(t)|}{1+|t|} d t<+\infty
$$

Consider the Cauchy transform of $f$ defined by

$$
(\mathcal{C} f)(\zeta) \stackrel{\text { def }}{=} \frac{1}{2 \pi \mathrm{i}} \int_{\mathbb{R}} \frac{f(t) d t}{t-\zeta}, \quad \operatorname{Im} \zeta \neq 0
$$


It is well known that $(\mathcal{C} f) \mid \mathbb{C}_{+}$and $(\mathcal{C} f) \mid \mathbb{C}_{-}$are holomorphic functions of bounded characteristic in $\mathbb{C}_{+}$and $\mathbb{C}_{-}$respectively, and so they have finite angular boundary values almost everywhere on $\mathbb{R}$. Set

$$
f_{+}(x) \stackrel{\text { def }}{=} \lim _{y \rightarrow 0+}(\mathcal{C} f)(x+\mathrm{i} y) \quad \text { and } \quad f_{-}(x) \stackrel{\text { def }}{=}-\lim _{y \rightarrow 0-}(\mathcal{C} f)(x+\mathrm{i} y) .
$$

By the Privalov theorem (see $[\mathrm{Pr}]$ for the case of a rectifiable Jordan curve), $f=f_{+}+f_{-}$almost everywhere on $\mathbb{R}$. If $f \in L^{1}(\mathbb{R})+L^{2}(\mathbb{R})$, then

$$
(\mathcal{C} f)(z)=\int_{0}^{\infty}(\mathcal{F} f)(t) e^{2 \pi i t z} d t, \quad z \in \mathbb{C}_{+},
$$

and

$$
(\mathcal{C} f)(z)=-\int_{-\infty}^{0}(\mathcal{F} f)(t) e^{2 \pi \mathrm{i} t z} d t, \quad z \in \mathbb{C}_{-} .
$$

Note that $f_{+}$does not have to be in $L^{1}(\mathbb{R})$ for an arbitrary function $f \in L^{1}(\mathbb{R})$. In fact, if $f \in L^{1}(\mathbb{R})$, then $f_{+} \in L^{1}(\mathbb{R})$ if and only if $f$ belongs to the Stein-Weiss space $\mathfrak{H}^{1}$.

Theorem 15.15. Let $f \in \mathfrak{H}^{1}$. Suppose that there exists a function $g \in L^{1}(\mathbb{R})$ such that $(\mathcal{F} f)(x)=(\mathcal{F} g)(x) \log \left(1+x^{2}\right)$ for all $x \in \mathbb{R}$. Then

$$
\lim _{t \rightarrow 0} \omega_{g_{+}}^{(1)}(t) \log \frac{1}{t}=0
$$

and

$$
\lim _{t \rightarrow 0} \omega_{g_{-}}^{(1)}(t) \log \frac{1}{t}=0 .
$$

Note, however, that the assumptions of Theorem 15.15 do not imply that $g_{+} \in L^{1}(\mathbb{R})$ or $g_{-} \in L^{1}(\mathbb{R})$.

We need some auxiliary facts. Let $\mathcal{M}(\mathbb{R})$ be the space of finite Borel measures on $\mathbb{R}$.

Lemma 15.16. Let $f \in L^{1}(\mathbb{R})$. Suppose that $f^{\prime \prime} \in \mathcal{M}(\mathbb{R})$ (in the distributional sense). Then $\mathcal{F} f \in L^{1}(\mathbb{R})$ and

$$
\|\mathcal{F} f\|_{L^{1}(\mathbb{R})} \leq C \sqrt{\|f\|_{L^{1}(\mathbb{R})}\left\|f^{\prime \prime}\right\|_{\mathcal{M}(\mathbb{R})}} .
$$

Proof. The result follows from the obvious inequality:

$$
|(\mathcal{F} f)(x)| \leq \min \left\{\|f\|_{L^{1}}, \frac{\left\|f^{\prime \prime}\right\|_{\mathcal{M}(\mathbb{R})}}{4 \pi^{2} x^{2}}\right\}, \quad x \in \mathbb{R} .
$$

Corollary 15.17. Let $f \in L^{1}(\mathbb{R})$. Suppose that $\operatorname{supp} \mathcal{F} f$ is bounded above. Then

$$
\omega_{f_{+}}^{(1)}(t) \leq \text { const } \cdot t, \quad t>0 .
$$


Proof. It suffices to construct a function $g_{s} \in L^{1}(\mathbb{R})$ such that $\left\|g_{s}\right\|_{L^{1}(\mathbb{R})} \leq C|s|$ and $f_{+}(x+s)-f_{+}(x)=\left(f * g_{s}\right)(x)$ for all $x \in \mathbb{R}$. Suppose that $\operatorname{supp} f \subset(-\infty, M]$, where $M>0$. We may take a function $g_{s}$ such that

$$
\left(\mathcal{F} g_{s}\right)(t)= \begin{cases}0, & t \leq 0, \\ e^{2 \pi \mathrm{i} s t}-1, & t \in[0, M], \\ e^{2 \pi \mathrm{i} s(2 M-t)}-1, & t \in[M, 2 M], \\ 0, & t \geq 2 M .\end{cases}
$$

Clearly, $f_{+}(x+s)-f_{+}(x)=\left(f * g_{s}\right)(x)$ for all $x \in \mathbb{R}$. The inequality $\left\|g_{s}\right\|_{L^{1}(\mathbb{R})} \leq C|s|$ follows from Lemma 15.16 (with $C$ depending on $M$ ).

Lemma 15.18. Set $\rho(t) \stackrel{\text { def }}{=} 2 \log (2+|t|)-\log \left(1+t^{2}\right)$. Then $\mathcal{F} \rho \in L^{1}$.

Proof. It suffices to observe that $\rho$ is even, $\lim _{t \rightarrow \infty} \rho(t)=0, \rho$ has two continuous derivatives on $(0, \infty)$, and

$$
\int_{0}^{\infty} t\left|\rho^{\prime \prime}(t)\right| d t<\infty ;
$$

this implies that $\rho(x)=-\int_{|x|}^{\infty} \rho^{\prime}(t) d t$ and

$$
\rho(x)=\int_{|x|}^{\infty}(t-|x|) \rho^{\prime \prime}(t) d t=\int_{0}^{\infty} \rho^{\prime \prime}(t)(t-|x|)_{+} d t=\int_{0}^{\infty} \rho^{\prime \prime}(t) t \mathcal{F} K_{t}(x) d t,
$$

where $K_{t}$ is the Fejér kernel with $\left\|K_{t}\right\|_{L^{1}}=1$.

Lemma 15.19. Let $\varphi$ be an even positive function in $C^{2}(\mathbb{R})$ such that $\varphi(x)=$ $\log \left(1+x^{2}\right)$ for sufficiently large $|x|$. Then $\mathcal{F}\left(\varphi^{-1}\right) \in L^{1}$.

Proof. See the proof of the previous lemma.

Proof of Theorem 15.15. We prove the first equality (the proof of the second one is the same). Let $\psi$ be a function in $L^{1}(\mathbb{R})$ such that $\operatorname{supp} \mathcal{F} \psi$ is compact and $\operatorname{supp} \mathcal{F} \psi=1$ in a neighborhood of 0 . Then $f=f * \psi+(f-f * \psi)$. The Fourier transform of the first summand has a compact support while the support of the Fourier transform of the second summand does not contain 0 . Thus it is sufficient to consider two cases.

Case 1, supp $\mathcal{F} f$ is compact. The result follows from Corollary 15.17.

Case 2, $0 \notin \operatorname{supp} \mathcal{F} f$. Clearly,

$$
\left(\mathcal{F} f_{+}\right)(x)=\left(\mathcal{F} g_{+}\right)(x) \log \left(1+x^{2}\right), \quad x \in \mathbb{R} .
$$

Let $\delta>0$ be such that $\mathcal{F} f=0$ on $[-\delta, \delta]$, and let $\varphi$ be an even positive function in $C^{2}(\mathbb{R})$ such that $\varphi(x)=\log \left(1+x^{2}\right)$ for $|x| \geq \delta$. By Lemma $15.19,1 / \varphi=\mathcal{F} \Phi$ for some $\Phi \in L^{1}(\mathbb{R})$. Hence,

$$
\mathcal{F} g_{+}=\mathcal{F} f_{+} / \varphi=\mathcal{F}\left(f_{+} * \Phi\right),
$$


which implies that $g_{+} \in L^{1}(\mathbb{R})$. Moreover, if $\rho$ is as in Lemma 15.18, and thus $\rho=\mathcal{F} F$ with $F \in L^{1}(\mathbb{R})$, then

$$
2\left(\mathcal{F} g_{+}\right)(x) \log (2+|x|)=\left(\mathcal{F} g_{+}\right)(x) \rho(x)+\left(\mathcal{F} f_{+}\right)(x)=\mathcal{F}\left(g_{+} * F+f_{+}\right)(x),
$$

so $\left(\mathcal{F} g_{+}\right)(x) \log (2+|x|)$ is the Fourier transform of an $L^{1}$-function. It remains to apply Theorem 15.14 .

Theorem 15.20. Let $\varphi$ be a function in $L^{2}[0, b]$ such that $Q_{\varphi}^{[0, b]} \in \boldsymbol{S}_{1}$ and let $\varphi(x)=\sum_{n \in \mathbb{Z}} a_{n} e^{2 \pi \mathrm{i} n x / b}$. If

$$
\phi_{+}(\zeta) \stackrel{\text { def }}{=} \sum_{n \geq 0} a_{n} \zeta^{n} \quad \text { and } \quad \phi_{-}(\zeta) \stackrel{\text { def }}{=} \sum_{n<0} a_{n} \zeta^{n}
$$

then

$$
\lim _{t \rightarrow 0} \omega_{\phi_{+}}^{(1)}(t) \log \frac{1}{t}=0 \quad \text { and } \quad \lim _{t \rightarrow 0} \omega_{\phi_{-}}^{(1)}(t) \log \frac{1}{t}=0 .
$$

Proof. The result follows immediately from Theorems 15.4 and 15.11.

Theorem 15.21. Let $I$ be a compact interval in $(0, \infty)$ and let $\varphi$ be a function in $L_{\mathrm{loc}}^{1}\left(\mathbb{R}_{+}\right)$such that $Q_{\varphi} \in \boldsymbol{S}_{1}$. If

$$
a_{n}=\int_{I} \varphi(x) e^{-2 \pi \mathrm{i} n x /|I|} d x, \quad n \in \mathbb{Z}
$$

and $\phi_{+}$and $\phi_{-}$are defined by (15.8), then (15.9) holds.

Proof. The result is an immediate consequence of Theorem 15.20.

Recall that for a function $\varphi \in L_{\text {loc }}^{2}\left(\mathbb{R}_{+}\right)$the function $\varphi^{\odot}$ is defined by (13.1). Note that if $Q_{\varphi} \in \boldsymbol{S}_{1}$, then by Theorem $6.2, \varphi \in L^{1}\left(\mathbb{R}_{+}\right)$and thus $\varphi^{\ominus} \in L^{1}(\mathbb{R})$.

Theorem 15.22. Let $\varphi$ be a function in $L_{\mathrm{loc}}^{2}\left(\mathbb{R}_{+}\right)$such that $Q_{\varphi} \in \boldsymbol{S}_{1}$. Then

$$
\lim _{t \rightarrow 0} \omega_{\left(\varphi^{\oplus}\right)_{+}}^{(1)^{\prime}}(t) \log \frac{1}{t}=0 \quad \text { and } \quad \lim _{t \rightarrow 0} \omega_{\left(\varphi^{\oplus}\right)_{-}}^{(1)}(t) \log \frac{1}{t}=0 .
$$

In particular,

$$
\lim _{t \rightarrow 0} \omega_{\varphi^{\odot}}^{(1)}(t) \log \frac{1}{t}=0 .
$$

Proof. The result follows from Theorem 13.12, Corollary 15.8, and Theorem 15.15.

This result should be compared to Theorems 10.7 and 13.7. In particular, if $\varphi$ has compact support in $\mathbb{R}_{+}$, we see that a Dini condition on the $L^{2}$ modulus of continuity is sufficient for $Q_{\varphi} \in \boldsymbol{S}_{1}$, while the slightly weaker condition $\lim _{t \rightarrow 0} \omega_{\varphi}^{(1)}(t) \log \frac{1}{t}=0$ on the $L^{1}$ modulus of continuity is necessary. 
Theorem 15.23. Let $\varphi$ be a function in $L_{\text {loc }}^{2}\left(\mathbb{R}_{+}\right)$such that $Q_{\varphi} \in \boldsymbol{S}_{1}$. Then

$$
\lim _{a \rightarrow 1} \int_{\mathbb{R}_{+}}|\varphi(a x)-\varphi(x)| d x \cdot \log \frac{1}{|a-1|}=0 .
$$

Proof. By Theorem 15.22, we have

$$
\lim _{t \rightarrow 0} \int_{\mathbb{R}}\left|\varphi\left(e^{2 s+2 t}\right) e^{2 s+2 t}-\varphi\left(e^{2 s}\right) e^{2 s}\right| d s \cdot \log \frac{1}{t}=0 .
$$

Substituting $e^{2 s}=x$ and $e^{2 t}=a$, we obtain

$$
\lim _{a \rightarrow 1} \int_{\mathbb{R}_{+}}|a \varphi(a x)-\varphi(x)| d x \cdot \log \frac{1}{|a-1|}=0 .
$$

It remains to observe that by Theorem $6.2, \varphi \in L^{1}\left(\mathbb{R}_{+}\right)$and obviously,

$$
\lim _{a \rightarrow 1}|a-1| \cdot \log \frac{1}{|a-1|}=0 \text {. }
$$

\section{Dilation of Symbols}

Let $\varphi$ be a function in $L_{\text {loc }}^{2}(\mathbb{R})$ such that $\varphi(x+1)=\varphi(x), x \in \mathbb{R}$. For $a>0$ we define the function $\varphi_{a}$ on $[0,1]$ by $\varphi_{a}(x) \stackrel{\text { def }}{=} \varphi(a x)$ for $x \in[0,1)$. We are going to obtain in this section upper and lower estimates for $\left\|Q_{\varphi_{a}}^{[0,1]}\right\|_{\boldsymbol{S}_{p}}$.

Note that we can extend $\varphi_{a}$ to $\mathbb{R}$ as a 1-periodic function on $\mathbb{R}$. Using an obvious estimate, see (14.1)-(14.3),

$$
C_{1}\left(\left\|Q_{\varphi_{a}}^{[0,1]}\right\|_{\boldsymbol{S}_{p}}+\left\|\varphi_{a}\right\|_{L^{2}[0,1]}\right) \leq\left\|Q_{\varphi_{a}}^{[0,2]}\right\|_{\boldsymbol{S}_{p}} \leq C_{2}\left(\left\|Q_{\varphi_{a}}^{[0,1]}\right\|_{\boldsymbol{S}_{p}}+\left\|\varphi_{a}\right\|_{L^{2}[0,1]}\right),
$$

we can reduce the estimation of $\left\|Q_{\varphi_{a}}^{[0,1]}\right\|_{\boldsymbol{S}_{p}}$ to that of $\left\|Q_{\varphi_{a}}^{[0,2]}\right\|_{\boldsymbol{S}_{p}}$. We can consider the Fourier coefficients of $\varphi_{a}$ defined by

$$
\hat{\varphi}_{a}(n) \stackrel{\text { def }}{=} \int_{0}^{1} \varphi_{a}(t) e^{-2 \pi \mathrm{in} t} d t, \quad n \in \mathbb{Z} .
$$

Theorem 16.1. Let $\varphi$ be a 1-periodic function in $L_{\mathrm{loc}}^{2}(\mathbb{R})$ and let a $>0$. Suppose that $\varphi$ has bounded variation on $[0,1]$. Then

$$
\left\|Q_{\varphi a}^{[0,2]}\right\|_{S_{1}} \leq C(\varphi) \log (2+a)
$$

and

$$
\left\|Q_{\varphi_{a}}^{[0,2]}\right\|_{\boldsymbol{S}_{p}} \leq C(\varphi)(1+a)^{1 / p-1}, \quad 1 / 2<p<1
$$


Proof. The result follows from Theorem 7.7.

Theorem 16.2. Let $\varphi$ be a nonconstant 1-periodic function in $L_{\text {loc }}^{2}(\mathbb{R})$. Then for $a \geq 1$

$$
\left\|Q_{\varphi_{a}}^{[0,1]}\right\|_{\boldsymbol{S}_{1}} \geq C(\varphi) \log (2+a) .
$$

Proof. It follows from (16.1) that it is sufficient to prove that

$$
\left\|Q_{\varphi a}^{[0,2]}\right\|_{\boldsymbol{S}_{1}} \geq C(\varphi) \log (2+a)
$$

First we consider the case where $a$ is an integer. There exists an integer $k \in \mathbb{Z} \backslash\{0\}$ such that $\hat{\varphi}(k) \neq 0$. By Corollary 15.6,

$$
\left|\hat{\varphi}_{a}(l)\right| \leq C(\log (2+|l|))^{-1}\left\|Q_{\varphi_{a}}\right\|_{\boldsymbol{S}_{1}} .
$$

Substituting $l=a k$, we obtain (16.2), since $\hat{\varphi}_{a}(a k)=\hat{\varphi}(k)$.

Let now $a$ be an arbitrary number in $(1, \infty)$. For any $\sigma \in[1,2]$ there exists $k_{\sigma} \in \mathbb{Z} \backslash\{0\}$ such that $\hat{\varphi}_{\sigma}\left(k_{\sigma}\right) \neq 0$. Consequently, there exists a neighborhood $U_{\sigma}$ of $\sigma$ such that $\hat{\varphi}_{\tau}\left(k_{\sigma}\right) \neq 0$ for any $\tau \in U_{\sigma}$. The first part of the proof allows us to obtain the required estimate for any $a>1$ such that $a / N \in U_{\sigma}$ for some positive integer $N$. To complete the proof, we can choose a finite subcover $U_{\sigma_{j}}$ of $[1,2]$.

Theorem 16.3. Let $\varphi$ be a nonconstant 1-periodic function in $L_{\mathrm{loc}}^{2}(\mathbb{R})$ and let $0<p<1$. Then for $a \geq 1$

$$
\left\|Q_{\varphi_{a}}^{[0,1]}\right\|_{S_{p}} \geq C(\varphi) a^{1 / p-1}
$$

Proof. It suffices to consider the case when $a$ is an even integer. The general case may be reduced to this special case in the same way as in the proof of Theorem 16.2. With any kernel $k$ on the square $[0,1)^{2}$ and any integer $n \geq 1$ we associate the kernel $k^{[n]}$ defined by

$$
k^{[n]}(x, y)=n^{2} \int_{\frac{j}{n}}^{\frac{j+1}{n}} \int_{\frac{l}{n}}^{\frac{l+1}{n}} k(t, s) d t d s, \quad \text { if } \quad x \in\left[\frac{j}{n}, \frac{j+1}{n}\right) \quad \text { and } \quad y \in\left[\frac{l}{n}, \frac{l+1}{n}\right) .
$$

Clearly, $\left\|k^{[n]}\right\|_{\boldsymbol{S}_{p}} \leq\|k\|_{\boldsymbol{S}_{p}}$ for any positive $p$ (recall that $\|k\|_{\boldsymbol{S}_{p}}$ means the $S_{p}$-norm (or quasinorm) of the integral operator with kernel $k$ ).

Suppose that

$$
\int_{0}^{1} \varphi(t) d t \neq 2 \int_{0}^{1} t \varphi(t) d t .
$$

Put $k_{n}(x, y) \stackrel{\text { def }}{=} \varphi(n \max \{x, y\})$ for $x, y \in[0,1)$. Clearly,

$$
\left\|k_{2 n}^{[n]}-k_{2 n}^{[2 n]}\right\|_{\boldsymbol{S}_{p 5}} \leq 2^{1 / p}\left\|k_{2 n}\right\|_{\boldsymbol{S}_{p}} .
$$


It is not hard to see that on $\left[\frac{j}{n}, \frac{j+1}{n}\right) \times\left[\frac{l}{n}, \frac{l+1}{n}\right)$

$$
k_{2 n}^{[n]}= \begin{cases}\int_{0}^{1} \varphi(t) d t, & j \neq l, \\ \frac{1}{2} \int_{0}^{1} \varphi(t) d t+\int_{0}^{1} t \varphi(t) d t, & j=l .\end{cases}
$$

Next, on $\left[\frac{j}{2 n}, \frac{j+1}{2 n}\right) \times\left[\frac{l}{2 n}, \frac{l+1}{2 n}\right)$

$$
k_{2 n}^{[2 n]}= \begin{cases}\int_{0}^{1} \varphi(t) d t, & j \neq l \\ 2 \int_{0}^{1} t \varphi(t) d t, & j=l .\end{cases}
$$

Thus, the kernel $k_{2 n}^{[n]}-k_{2 n}^{[2 n]}$ vanishes outside the "diagonal" $\bigcup_{j=0}^{n-1}\left[\frac{j}{n}, \frac{j+1}{n}\right) \times\left[\frac{j}{n}, \frac{j+1}{n}\right)$.

Clearly, for $x, y \in\left[0, \frac{n-1}{n}\right) \times\left[0, \frac{n-1}{n}\right)$ we have

$$
k_{2 n}^{[n]}(x, y)-k_{2 n}^{[2 n]}(x, y)=k_{2 n}^{[n]}\left(x+\frac{1}{n}, y+\frac{1}{n}\right)-k_{2 n}^{[2 n]}\left(x+\frac{1}{n}, y+\frac{1}{n}\right) .
$$

Consequently,

$$
\left\|k_{2 n}^{[n]}-k_{2 n}^{[2 n]}\right\|_{\boldsymbol{S}_{p}}=n^{1 / p}\left\|\left(k_{2 n}^{[n]}-k_{2 n}^{[2 n]}\right) \chi_{\left[0, \frac{1}{n}\right) \times\left[0, \frac{1}{n}\right)}\right\|_{\boldsymbol{S}_{p}} .
$$

We have

$$
k_{2 n}^{[n]}-k_{2 n}^{[2 n]}=\frac{1}{2} \int_{0}^{1} \varphi(t) d t-\int_{0}^{1} t \varphi(t) d t
$$

on $\left(\left[0, \frac{1}{2 n}\right) \times\left[0, \frac{1}{2 n}\right)\right) \cup\left(\left[\frac{1}{2 n}, \frac{1}{n}\right) \times\left[\frac{1}{2 n}, \frac{1}{n}\right)\right)$ and

$$
k_{2 n}^{[n]}-k_{2 n}^{[2 n]}=\int_{0}^{1} t \varphi(t) d t-\frac{1}{2} \int_{0}^{1} \varphi(t) d t
$$

on $\left(\left[0, \frac{1}{2 n}\right) \times\left[\frac{1}{2 n}, \frac{1}{n}\right)\right) \cup\left(\left[\frac{1}{2 n}, \frac{1}{n}\right) \times\left[0, \frac{1}{2 n}\right)\right)$. Now it is easy to see that

$$
\left\|k_{2 n}^{[n]}-k_{2 n}^{[2 n]}\right\|_{S_{p}}=c n^{1 / p-1}
$$

for some nonzero $c$, since $\int_{0}^{1} \varphi(t) d t \neq 2 \int_{0}^{1} t \varphi(t) d t$.

Suppose now that $\varphi$ is an arbitrary nonconstant 1-periodic function. It suffices to prove that there exists $b \in \mathbb{R}$ such that $\int_{0}^{1} \varphi(t-b) d t \neq 2 \int_{0}^{1} t \varphi(t-b) d t$. Suppose 
that

$$
\int_{0}^{1} \varphi(t-b) d t=2 \int_{0}^{1} t \varphi(t-b) d t, \quad b \in \mathbb{R} .
$$

Let $h$ be the 1-periodic function such that $h(t)=2 t-1$ for $t \in[0,1)$. Clearly, $\hat{h}(n) \neq 0$ for $n \neq 0$. Thus it follows from (16.3) that $\varphi$ is constant.

\section{Acknowledgement}

This research was begun by the first and third authors. The two other authors joined them during the Conference on Function Spaces, Interpolation Theory, and related topics in honour of Jaak Peetre on his 65th birthday at Lund University, Sweden, August 17-22, 2000, where three of the authors were present and several of the results were proved. We thank Jonathan Arazy and Vladimir Maz'ya for helpful comments.

\section{References}

[A] A.B. Aleksandrov, Toeplitz Schur multipliers of $S_{p}\left(L^{2}(G)\right)$, Preprint, IHES, 2000

[AO] A.B. Alekseev and V.L. Oleinik, Estimates for widths of the unit ball of $\stackrel{\circ}{L}_{p}^{l}$ in $L_{p}(\mu)$ (Russian), in: Problems of mathematical physics, No. 7 (Russian), Leningrad University, $1974,3-7$.

[BL] J. Bergh and J. LÖfström, Interpolation spaces, Springer-Verlag, Berlin-HeidelbergNew York, 1976.

[B] M.Sh. Birman, A proof of the Fredholm trace formula as an application of a simple embedding for kernels of integral operators of trace class in $L^{2}\left(\mathbb{R}^{m}\right)$, Preprint, Dept. Math., Linköping University, LITH-MAT-R-89-30, 1989/90.

[BS] M.Sh. Birman and M.Z. Solomyak, Spectral theory of selfadjoint operators in Hilbert space, Mathematics and its Applications (Soviet Series), D. Reidel Publishing Co., Dordrecht, 1987.

[C] A. Connes, Noncommutative geometry, Academic Press, San Diego, CA, 1994.

[D] J. Dixmier, Existence de traces non normales (French), C. R. Acad. Sci. Paris Sér. A-B, 262 (1966), A1107-A1108.

[EEH] D.E. Edmunds, W.D. Evans, And D.J. Harris, Two-sided estimates for the approximation numbers of certain Volterra integral operators, Studia Math. 124 (1997), 59-80.

[ES] D.E. Edmunds And V.D. STePanov, On the singular numbers of certain Volterra integral operators, J. Funct. Anal. 134 (1995), no. 1, 222-246.

[GK1] I.C. GohberG And M.G. Krein, Vvedenie v teoriyu lineinykh nesamosopryazhyonnykh operatorov (Russian), Nauka, Moscow, 1965; English transl.: Introduction to the theory of linear nonselfadjoint operators in Hilbert space, Amer. Math. Soc., Providence, R.I., 1969. 
[GK2] I.C. Gohberg AND M.G. KREIn, Teoriya Vol'terrovykh ooperatorov v gil'bertovom prostranstve i eyo prilozheniya (Russian), Nauka, Moscow, 1967; English transl.: Theory and applications of Volterra operators in Hilbert space, Amer. Math. Soc., Providence, R.I., 1970.

[K] N.J. Kalton, Linear operators on $L^{p}$ for $0<p<1$, Trans. Amer. Math. Soc. 259 (1980), 319-355.

[LS] B.M. Levitan And I.S. SARgsjan, Operatory Shturma-Liuvillya i Diraka (Russian), Nauka, Moscow, 1988; English transl.: Sturm-Liouville and Dirac operators, Kluwer, Dordrecht, 1991.

[MV] V.G. MAZ'YA AND I.E. VERBITSKY, The Schrödinger operator on the energy space: boundedness and compactness criteria, Preprint, 2000.

[NaS] K. Naimark And M. Solomyak, Regular and pathological eigenvalue behavior for the equation $\lambda u^{\prime \prime}=V u$ on the semiaxis, Integr. Equat. Oper. Th. 20 (1994), 335-349.

[NeS] J. Newman AND M. Solomyak, Two-sided estimates on singular values for a class of integral operators on the semi-axis, J. Funct. Anal. 151 (1997), 504-530.

[No] K. NowAK, Schatten ideal behavior of a generalized Hardy operator, Proc. Amer. Math. Soc. 118 (1993), 479-483.

[OP] V.L. OlEINIK AND B.S. PAVlOV, On criteria of boundedness and complete continuity of certain imbedding operators (Russian), in: Problems of Mathematical Physics, No. 4: Spectral Theory. Wave Processes (Russian), Leningrad University, 1970, 112-117.

[Pee] J. Peetre, New thoughts on Besov spaces, Duke Univ. Press., Durham, N.C., 1976.

[Pel1] V.V. PELLER, Hankel operators of class $\mathbf{S}_{p}$ and their applications (rational approximation, Gaussian processes, the problem of majorizing operators) (Russian), Mat. Sbornik 41 (1980), 538-581. English transl. in Math. USSR Sbornik 41 (1982), 443-479.

[Pel2] V.V. PELLER, A description of Hankel operators of class $\mathbf{S}_{p}$ for $p>0$, an investigation of the rate of rational approximation, and other applications (Russian), Mat. Sbornik 122 (1983), 481-510. English transl. in Math. USSR Sbornik 50 (1985), 465-494.

[Pel3] V.V. PELlER, Metric properties of the averaging projection onto the set of Hankel matrices (Russian), Dokl. Akad Nauk SSSR 278 (1984), 271-285. English transl. in Soviet Math. Dokl. 30 (1984), 362-368.

[Pr] I.I. PRIVALOV, Granichnye svoistva analiticheskikh funktsii (Russian) [Boundary properties of analytic functions], 2nd ed., GITTL, Moscow-Leningrad, 1950. German transl.: Randeigenschaften analytischer Funktionen, VEB Deutscher Verlag, Berlin, 1956.

[RW] R. Rochberg And Z. Wu, A new characterization of Dirichlet type spaces and applications. Illinois J. Math. 37 (1993), no. 1, 101-122.

[R] S.Yu. RotFEL'D, On singular numbers of the sum of compact operators (Russian), in: Problems of Mathematical Physics, No. 3: Spectral Theory (Russian), Leningrad University, $1968,81-87$.

[Sch] L. Schwartz, Théorie des distributions (French), Hermann, Paris, 1966.

[St] V.D. Stepanov, On the lower bounds for Schatten-von Neumann norms of certain Volterra integral operators, J. London Math. Soc. (2) 61 (2000), no. 3, 905-922.

[W1] Z. Wu, Hankel and Toeplitz operators on Dirichlet spaces, Integral Equations Operator Theory 15 (1992), no. 3, 503-525.

[W2] Z. Wu, A class of bilinear forms on Dirichlet type spaces, J. London Math. Soc. (2) 54 (1996), no. 3, 498-514.

[Z] A. Zygmund, Trigonometric series, 2nd ed., Cambridge Univ. Press, London-New York, 1968. 
Alexei B. Aleksandrov, St-Petersburg Branch, Steklov Institute of Mathematics, Fontanka 27, 191011 St-Petersburg, Russia

E-mail address: alex@pdmi.ras.ru

Svante Janson, Department of Mathematics, Uppsala University, 75106 UppSALA, SWEDEN

E-mail address: svante@math.uu.se

Vladimir V. Peller, Department of Mathematics, Kansas State University, Manhattan, Kansas 66506, USA

E-mail address: peller@math.ksu.edu

Richard Rochberg, Department of Mathematics, Washington University, St. Louis, MO 63130, USA

E-mail address: rr@math.wustl.edu 